University of Louisville

ThinkIR: The University of Louisville's Institutional Repository

Electronic Theses and Dissertations

$8-2020$

\title{
Fatalism as a cultural influence on correlates of anxiety and worry in Latino/a adolescents.
}

Judy Mier-Chairez

University of Louisville

Follow this and additional works at: https://ir.library.louisville.edu/etd

Part of the Child Psychology Commons, Clinical Psychology Commons, and the Multicultural Psychology Commons

\section{Recommended Citation}

Mier-Chairez, Judy, "Fatalism as a cultural influence on correlates of anxiety and worry in Latino/a adolescents." (2020). Electronic Theses and Dissertations. Paper 3521.

https://doi.org/10.18297/etd/3521

This Doctoral Dissertation is brought to you for free and open access by ThinkIR: The University of Louisville's Institutional Repository. It has been accepted for inclusion in Electronic Theses and Dissertations by an authorized administrator of ThinkIR: The University of Louisville's Institutional Repository. This title appears here courtesy of the author, who has retained all other copyrights. For more information, please contact thinkir@louisville.edu. 


\title{
FATALISM AS A CULTURAL INFLUENCE ON CORRELATES OF ANXIETY AND
} WORRY IN LATINO/A ADOLESCENTS

\author{
By \\ Judy Mier-Chairez \\ B.A., University of Colorado, 2013 \\ M.S., University of Louisville, 2017

\begin{abstract}
A Dissertation
Submitted to the Faculty of the

College of Arts and Sciences of the University of Louisville in Partial Fulfillment of the Requirements

for the Degree of
\end{abstract} \\ Doctor of Philosophy \\ in Clinical Psychology \\ Department of Psychological and Brain Sciences \\ University of Louisville \\ Louisville, Kentucky
}

August 2020 

FATALISM AS A CULTURAL INFLUENCE ON CORRELATES OF ANXIETY AND WORRY IN LATINO/A ADOLESCENTS

\author{
By \\ Judy Mier-Chairez \\ B.A., University of Colorado, 2013 \\ M.S., University of Louisville, 2017 \\ A Dissertation Approved on
}

July 14,2020

by the following Dissertation Committee:

Janet Woodruff-Borden, Ph.D.

Bernadette Walter, Ph.D.

Richard Lewine, Ph.D.

Paul Salmon, Ph.D.

Lisa M. Hooper, Ph.D. 


\section{DEDICATION}

This dissertation is dedicated to the Mier and Ramos families that I proudly represent and without whose perseverance, wisdom, and unconditional support none of this would be possible. 


\section{ACKNOWLEDGMENTS}

I would like to thank my cherished mentor, Dr. Janet Woodruff-Borden, who is my role model and the constant that kept me grounded and continuously striving. Thank you to Dr. Bernadette Walter who has always believed in me; to Dr. Rich Lewine, for his thoughtfulness and insightful questions that undoubtedly added depth to the project; to Dr. Paul Salmon, for his expertise and support; and to Dr. Lisa Hooper, for her guidance and patience with me as a brand new graduate student. Thank you to Tim and Bailey Ray, for their encouragement and for being my refuge. Finally, thank you to Chelsea Siwik, Nonah Olesen, Catherine McDermott, Colette Gramszlo, and Angela Lee for their friendship, laughs, and solidarity. 


\section{ABSTRACT \\ FATALISM AS A CULTURAL INFLUENCE ON CORRELATES OF ANXIETY AND WORRY IN LATINO/A ADOLESCENTS}

Judy Mier-Chairez

July 14,2020

Evidence that Latino/as in the United States experience lower rates of anxiety disorders as compared to the non-Latino/a White population has not yet led to the identification of the sources of these differences. Higher rates of anxiety disorders among more acculturated Latino/a individuals compared to those lower in acculturation suggest there are influential cultural variables relevant to anxiety, specifically that there is a loss of a protective Latino/a cultural factor in the acculturation process. Fatalism, an often-cited Latino/a cultural characteristic, emerges as an intriguing candidate for exploration in relation to anxiety due to the shared elements of future orientation and control. Specifically, anxiety correlates of intolerance of uncertainty and primary and secondary control were studied. The current study reviews literature on cultural factors relevant to the Latino/a experience and their role in the developmental period of adolescence, anxiety in the Latino/a population, and the construct of fatalism and its role in Latina/o health and mental health. Next, the relations between acculturation, fatalism, and correlates of anxiety are tested. Specifically, it was hypothesized acculturation 
predicts fatalism, fatalism negatively predicts anxiety, worry, IU, and primary control, and positively predicts secondary control. Furthermore, it was hypothesized secondary control would moderate the relation between fatalism and anxiety and worry that higher fatalism will predict lower levels of anxiety and worry when a high level of secondary control is present. 47 Latino/a participants ages $12-17$ were recruited for the study. The adolescents and their parent/guardian completed self-report questionnaires. The study hypotheses were partially supported. Initial analyses indicated acculturation did not predict fatalism; however, exploratory analyses using a four-part model of acculturation revealed a significant interaction such that different combinations of high and low Hispanicism and Americanism related differently to fatalism. Fatalism was not a significant predictor of anxiety but was found to be a positive predictor of worry. Fatalism positively predicted IU for a subset of older adolescents. Finally, fatalism was not significantly predictive of primary or secondary control; however, secondary was a significant moderator in the relation between fatalism and anxiety. Implications of the findings for the future study of Latino/a anxiety are discussed. 


\section{TABLE OF CONTENTS}

\section{PAGE}

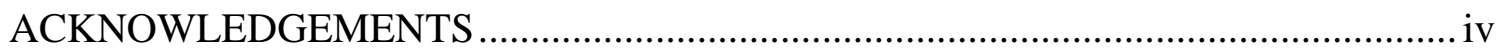

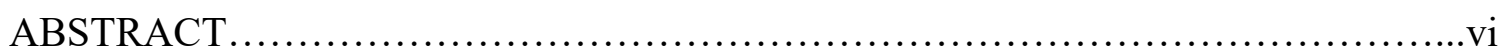

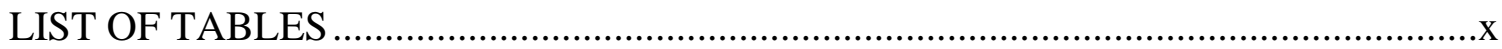

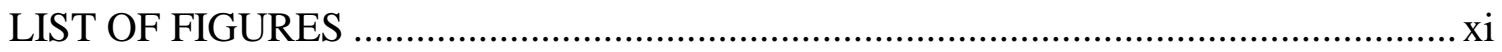

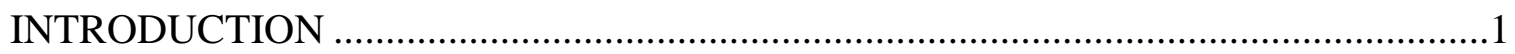

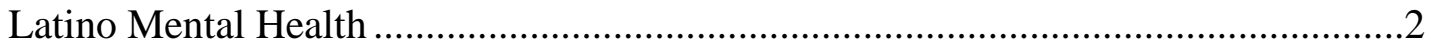

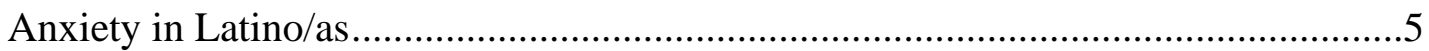

Latino/a cultural presentations of anxiety ......................................................

Hispanic health paradox...............................................................................

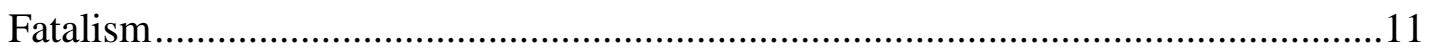

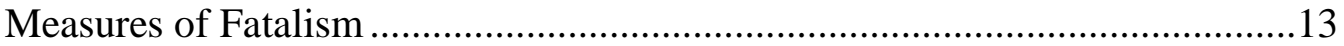

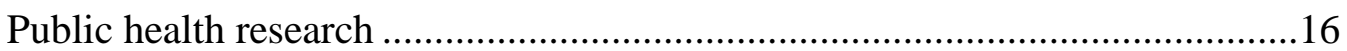

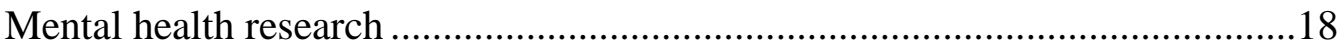

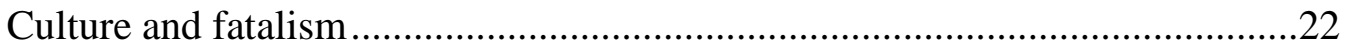

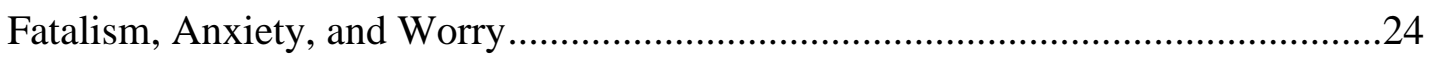

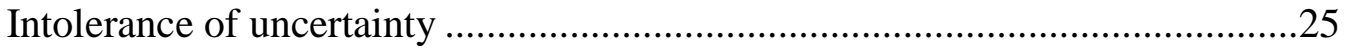

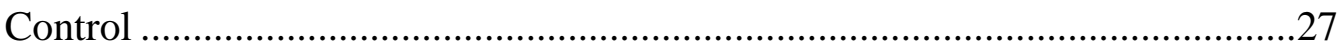

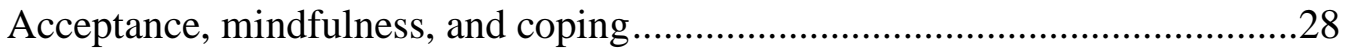

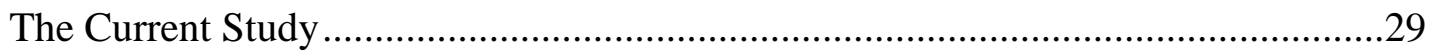




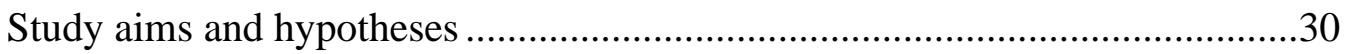

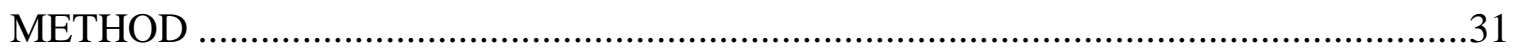

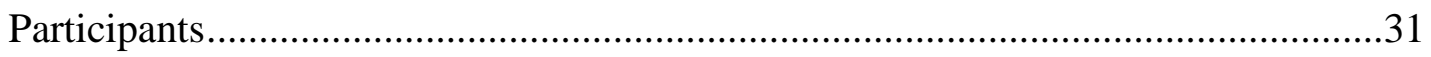

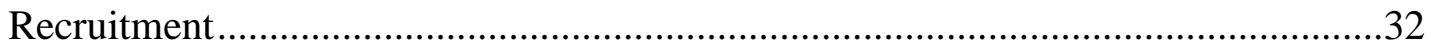

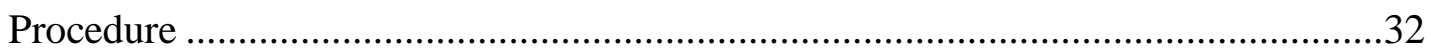

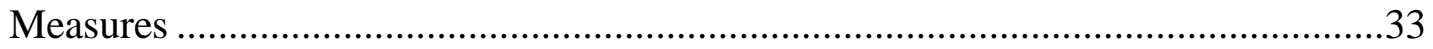

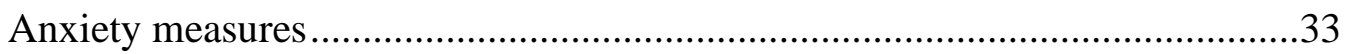

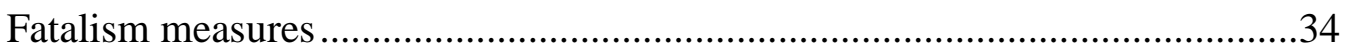

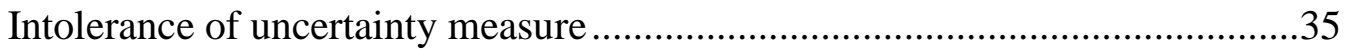

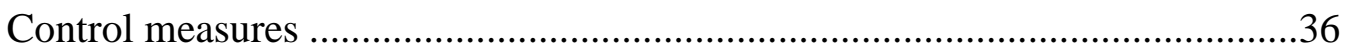

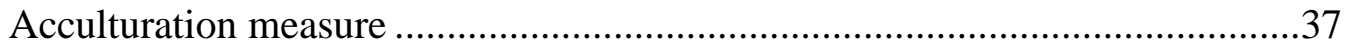

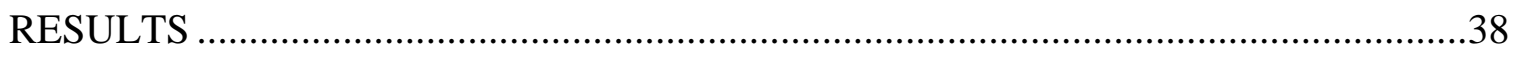

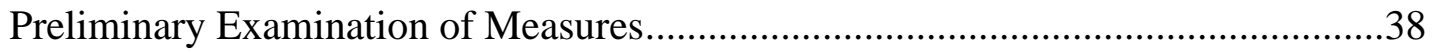

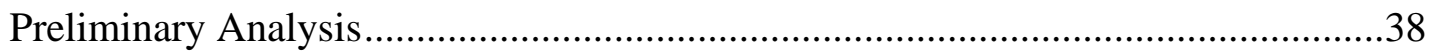

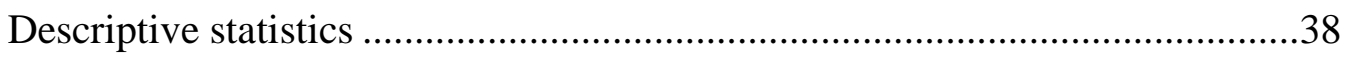

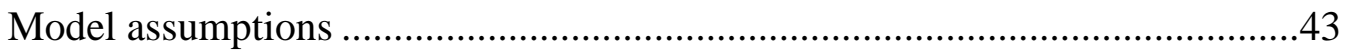

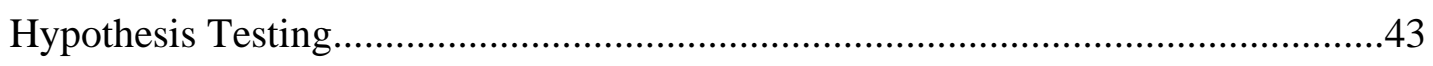

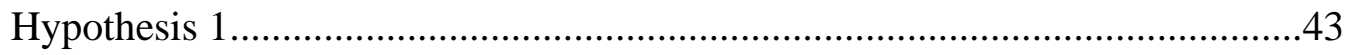

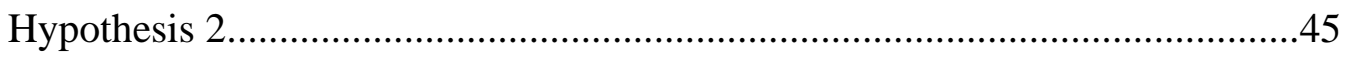

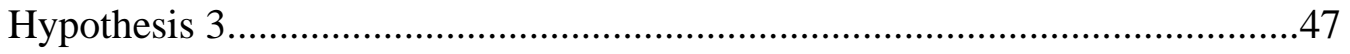

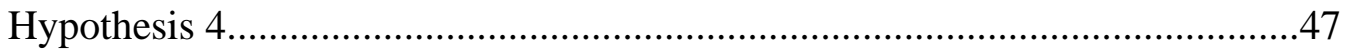

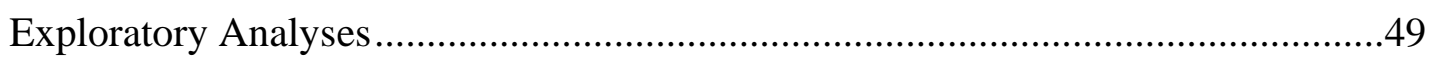

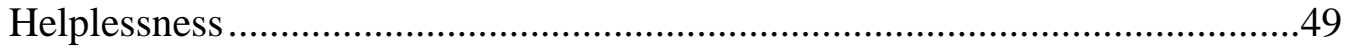


Four acculturation groups and fatalism................................................50

Four acculturation groups and anxiety and worry ....................................52

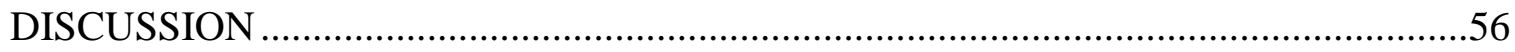

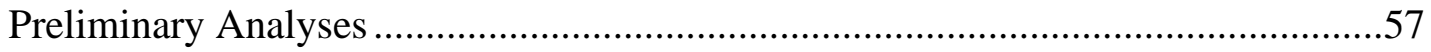

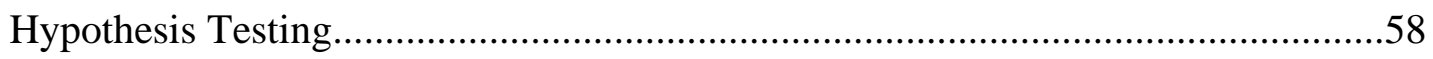

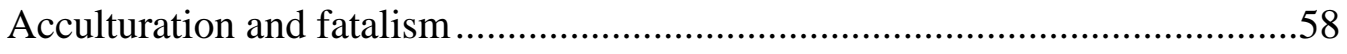

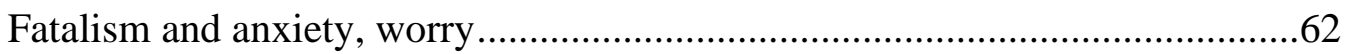

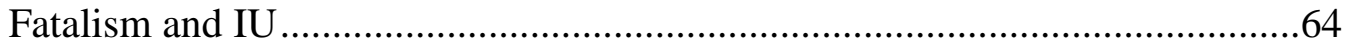

Fatalism and control, anxiety, worry …..............................................65

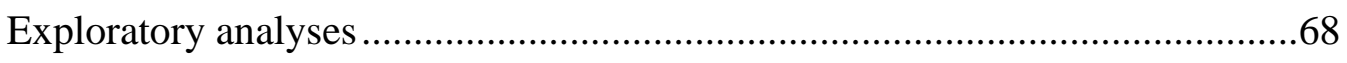

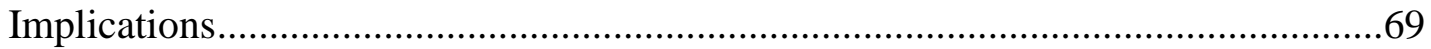

Limitations of the Current Study ............................................................. 70

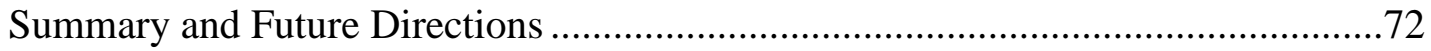

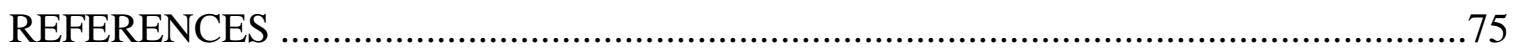

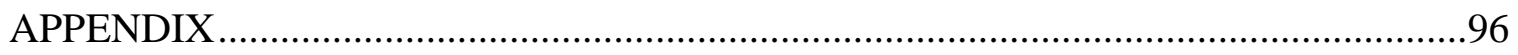

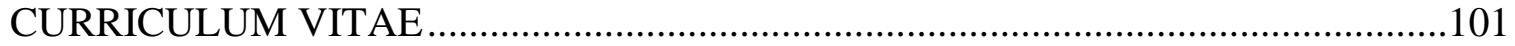




\section{LIST OF TABLES}

TABLE

PAGE

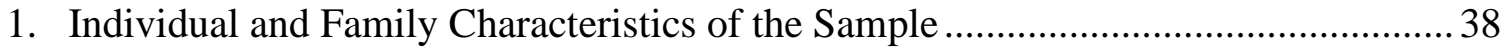

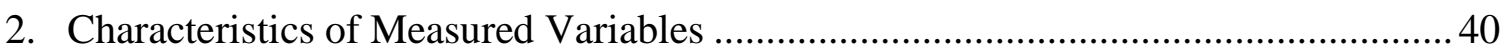

3. Correlation Coefficients Between BIQ and MFS subscales ................................... 44

4. Correlation Coefficients Between Fatalism, Anxiety Measures, and Covariates ....... 46

5. Correlation Coefficients between Fatalism and Control Measures .......................... 47

6. Correlation Coefficients between Control and Anxiety Measures ........................... 48

7. Results of Regression Analyses for Helplessness Predicting Outcome Variables ..... 50

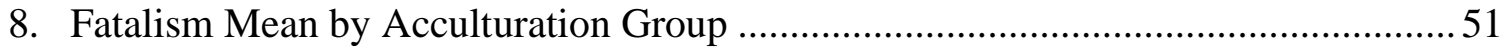

9. Anxiety Mean by Acculturation Group ................................................................ 53

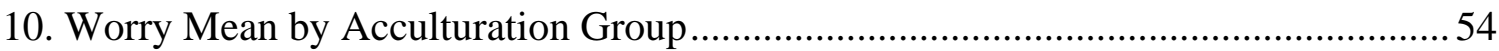




\section{LIST OF FIGURES}

1. Secondary Control Moderates Relation of Fatalism and Anxiety ............................49

2. Interaction of Americanism and Hispanicism Levels on Fatalism ............................52

3. Interaction of Americanism and Hispanicism Levels on Anxiety ............................54

4. Interaction of Americanism and Hispanicism Levels on Worry ...............................55

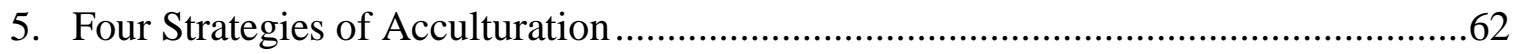




\section{INTRODUCTION}

As a steadily and rapidly growing population in the United States, Latino/as comprise a sizable demographic in today's society within the U.S. Estimates by the United States Census Bureau report that Latino/as constituted 17.3\% of the U.S. population in 2016 (U.S. Census Bureau, 2016). Though not explicitly stated, this presumably includes immigrants, citizens and non-citizens, first generation U.S.-born Latino/as, and later generations. The large majority of the U.S. Latino/a population is of Mexican descent (64\%), followed by Puerto Rican descent (10\%), and Cuban descent (4\%). Latino/as bring with them a culture that is unique to many communities within the U.S. With increased contact and interaction with this culture comes an interest in understanding how Latino/as think and behave and how that fits within the extant U.S. culture.

The study of Latino/a mental health is timely given the influx of stressors many Latino/as are facing in the U.S. today. Recently, the fear of deportation has been heightened for Latino/as in the U.S. This fear is not limited to undocumented immigrants or even immigrants at all and is shared by entire families and communities regardless of documentation status (Hacker et al., 2011). Drawing from the diathesis stress model, it can be predicted these increasing stressors will present as an upsurge in psychopathology generally, and potentially worry-based anxiety disorders specifically, for Latino/as (Gazelle \& Ladd, 2003) who had previously, as a group, demonstrated lower rates of 
anxiety disorders in cross-cultural studies (Asnaani, Richey, Dimaite, Hinton, \& Hofmann, 2010). A recent study by Salas-Wright and colleagues (2015) supports this prediction with a finding that, in their sample of Latino/as immigrants, individuals with a fear of deportation were more likely to meet criteria for generalized anxiety disorder than were individuals with no acculturative stress or fear of deportation.

A review of the literature on Latino/a mental health, anxiety and worry specifically, elucidates a large area of underdeveloped research and missing empirical connectors. Foundational research finding an overall lower prevalence of anxiety disorders in Latino/as as compared to non-Latino/a White Americans, and the trajectory it follows with acculturation, has not yet developed into identifying the influential cultural variables that serve as the mechanisms of this phenomenon. The potential cultural influences are plentiful, but fatalism emerges as an intriguing candidate for exploration. The shared elements of attitude toward uncertainty, perceptions of control, and acceptance conceptually connect fatalism, anxiety, and worry. To support the rationale for examining fatalism and its role in anxiety and worry in Latino/a adolescents, I will review cultural factors relevant to the Latino/a experience and their role in the developmental period of adolescence, anxiety in the Latino/a population, the construct of fatalism and its role in Latina/o health and mental health. I will then integrate the review with a conceptual summary and hypotheses of how fatalism can inform extant models of anxiety and worry in Latino/as, concluding with a detailed description of the current study. 


\section{LATINO/A MENTAL HEALTH}

Despite the important differences within the Latino/a demographic, there is evidence of shared cultural characteristics aside from shared language and somewhat similar physical features. Over the years through qualitative and quantitative study, a number of central cultural characteristics or values have been identified. These characteristics include familism, personalismo, simpatía, respeto, machismo, marianismo, and fatalism, among others (Ho, 1987; Ruiz et al., 2016; Soto et al., 2011). These characteristics have been studied empirically, to varying degrees, and have demonstrated unique relationships with psychopathology. For example, the heightened importance of family, or familism, was evident in a study of Latina women in which findings indicated negative interactions with family was predictive of generalized anxiety disorder whereas family cohesion protected against the disorder (Ai, Weiss, \& Fincham, 2014). Familism has also been studied in relation to depression, acculturative stress, and externalizing problems in adolescents to name a few (Kapke, Grace, Gerdes, \& Lawton, 2017; UmañaTaylor, Updegraff, \& Gonzales-Backen, 2011; Zeiders et al., 2013). Machismo and marianismo have been topics of interest as well in studies assessing the impact of these cultural characteristics on mental health. For example, a small online survey study $(n=$ 45) of Latino veteran men found a positive association between machismo and posttraumatic stress disorder (Herrera, Owens, \& Mallinckrodt, 2013).

Fatalism or fatalismo, is the central topic of the current study. Fatalism is consistently cited as a core descriptor of Latino/a culture but has less frequently been studied in-depth or been connected to health outcomes, particularly mental health outcomes. Briefly, fatalism is defined as a belief that the future is determined by fate 
(Espinosa de los Monteros \& Gallo, 2011). This culturally propagated belief is of relevance when investigating Latino/a mental health outcomes, anxiety specifically, due to its theme of future orientation and control (e.g., Piña-Watson \& Abraído-Lanza, 2017; Roncancio, Ward, \& Berenson, 2011).

Adolescence is of particular interest in relation to cultural influences on anxiety and worry because of the importance of identity development at this age and the high prevalence of anxiety disorders in adolescents (Costello, Egger, \& Angold, 2005; Schwartz et al., 2015). Latino/as in the United States of all ages face the challenge of integrating multiple cultures into their identity. Previously, this had been viewed as a unidimensional process in which there existed one spectrum with Latino identity on one end and American identity on the other. More recently, researchers have argued that a bicultural identity model is more accurate (Smokowski, Buchanan, \& Bacallao, 2009). Bicultural identity includes two spectrums, one for Latino identity and one for American identity. This means that a person can both identify greatly with being Latino and with being American simultaneously. Adolescence is an age at which many of these questions are at the forefront.

As a teen is developing their sense of autonomy and unique identity, they are making choices about important aspects of their life. In choosing the characteristics by which they would like to be recognized, they are inevitably making choices about their cultural identity. For example, they may be deciding, if ambiguous, name pronunciation, whether to foster bilingualism, the ethnic make-up of their friend group, and how often they attend Latino/a-centered events with their family. Potentially depending on their relationships with their families and with their peers, their experiences of peer rejection 
or bullying, and the availability of Latino/a peers, an adolescent may choose to distance themselves from the Latino/a culture if they perceive it will be socially beneficial. The question remains, however, if this puts the Latino/a adolescent at risk for poor mental health outcomes generally, and elevated anxiety and worry specifically (Smokowski et al., 2009).

\section{ANXIETY IN LATINO/AS}

Epidemiological research on the rates of psychopathology in the Latino/a population has elucidated significant differences in prevalence across a number of disorders. Notably, Alegría and colleagues (2008) analyzed results of two large-scale surveys for comparison of lifetime prevalence of psychopathology between Latino/as and non-Latino/a White individuals. This study drew from the data of the National Comorbidity Survey Replication (NCS-R) and National Latino and Asian American Study (NLAAS). The NCS-R is a large-scale survey that was conducted from February 2001 to April 2003 across the United States. The survey was conducted via in-person and telephone interviews and included only English-speaking adults in the U.S. Data from the NLAAS was collected from May 2002 to November 2003 and, as suggested by the name, looked to specifically target the Latino/a and Asian American populations. The NLAAS also only surveyed adults but included Spanish-speakers in addition to English-speakers. The Latino/a sample of the NLAAS consisted of 2,554 individuals. These two surveys were part of the NIMH's Collaborative Psychiatric Epidemiology Survey (CPES) program and assessed a multitude of variables generally under the category of risk factors for psychopathology. 
Analysis of the combined data found evidence of a lower lifetime prevalence of psychiatric illness overall in the Latino/a population as compared to the non-Latino/a White population (29.7\% and $43.2 \%$ respectively). This includes findings of lower rates of depressive disorders, substance-related disorders, and most anxiety disorders, with the exception of Agoraphobia without Panic Disorder. Specifically, Latino/as endorsed a lifetime prevalence of anxiety disorders at $15.7 \%$ whereas non-Latino/a White individuals endorsed a rate of $25.7 \%$. Importantly, this data was collected and analyzed prior to the DSM-5 changes to anxiety disorder classification and therefore included posttraumatic stress disorder along with panic disorder, agoraphobia without panic disorder, generalized anxiety disorder, and social anxiety disorder.

Asnaani, Richey, Dimaite, Hinton, and Hofmann (2010) expanded upon the analyses by Alegría and colleagues (2008) by adding the National Study of American Life (NSAL) to the data from the NCS-R and NLAAS. The NSAL is a large-scale household survey, also part of the CPES, with 6,199 respondents of African American and non-Latino/a White ethnic background. By adding the NSAL, Asnaani and colleagues (2010) were able to compare rates of psychopathology across multiple ethnic groups in contrast to Alegría and colleagues (2008) who only looked to study Latino/as with non-Latino/a White individuals as a reference group. The findings of this expanded analyses echoed that of the Alegría et al. (2008) study finding Latino/as endorsed lower rates of panic disorder, generalized anxiety disorder, social anxiety disorder, and posttraumatic stress disorder than non-Latino/a White individuals. Latino/as did not, however, have the lowest rate of these disorders across all ethnic groups. Latino/as endorsed higher rates of panic disorder and generalized anxiety disorder than African 
Americans and Asian Americans, and a higher rate of posttraumatic stress disorder than Asian Americans. This suggests that although Latino/as endorse lower rates of anxiety and other disorders than non-Latino/a White individuals, they are not especially immune to the disorders placing an important bound on the investigation of potential cultural protective factors unique to Latino/as.

Findings of lower prevalence of disorders can be interpreted in more than one way. The simplest interpretation would be to see the findings as a true reflection of experience and as evidence of a lower rate of these disorders. Another interpretation, however, may be that findings of lower rates is due to a difference in presentation and/or understanding that eludes our current measurement tools. For example, as discussed in the following section, there are certain culturally-bound presentations of anxiety that may not be accounted for in standard assessments of anxiety. More specifically, being that Ataque de Nervios is not always fear-based (Keough, Timpano, \& Schmidt, 2009), it may be difficult to capture it using our traditional methods and in fact likely needs to be specifically screened for.

Some evidence also supports the notion that differences are an artifact of measurement in research finding Latino/as are more likely to experience somatic symptoms of psychological disorders. In a study comparing the Beck Anxiety Inventory (BAI; Beck, Epstein, Brown, \& Steer, 1988), the Penn State Worry Questionnaire (PSWQ; Meyer, Miller, Metzger, \& Borkovec, 1990), the Anxiety Sensitivity Scale (ASI; Peterson \& Reiss, 1993), the Body Sensations Questionnaire (BSQ; Chambless, Caputo, Bright, \& Gallagher, 1984), and the State-Trait Personality Inventory- Trait Anxiety subscale (STPI-TA; Spielberger et al., 1979), measurement tools commonly used to 
assess anxiety and related constructs, Hirai, Stanley, and Novy (2006) found the BAI was the best predictor of severity of generalized anxiety disorder for Latino/as as diagnosed using the ADIS- IV (Brown, Di Nardo, \& Barlow, 1994). The authors suggest these findings indicate the more physiological focus of the BAI better captures the experience of anxiety and worry for Latino/as. In contrast, measurement tools that are more heavily focused on cognitive features may be failing to appropriately pick up on elevated anxiety and worry symptoms.

Latino/a cultural presentations of anxiety. Importantly, a robust collection of research has demonstrated, in addition to anxiety disorders as we understand them, Latino/as have unique culturally-specific or culturally-bound presentations of anxiety. The most well-recognized of these is ataque de nervios, which translates to "an attack of nerves," and is included in the DSM-5 under "Other Specified Anxiety Disorders." Ataque de nervios is characterized by "symptoms of intense emotional upset, including acute anxiety, anger, or grief; screaming and shouting uncontrollably; attacks of crying; trembling; heat in the chest rising into the head; and becoming verbally and physically aggressive" (American Psychiatric Association, 2013, p. 833). Ataque de nervios has been linked to panic however evidence suggests it is distinct both in the experience of it and in the etiology (Keough, Timpano, \& Schmidt, 2009; Lewis-Fernández et al., 2002). According to data from the NLAAS, $7-15 \%$ of the Latino/a sample had experienced ataque de nervios. A lesser-known cultural presentation of anxiety in Latino/as is susto, which translates to "fright," specifically referring to a frightening event or trauma an individual may have experienced that they believe disrupted something core to their self (Bayles \& Katerdahl, 2009). Susto is also in the DSM-5, under the "Glossary of Cultural 
Concepts of Distress" (American Psychiatric Association, 2013, p. 836), and has received empirical support in a smaller number of studies (Baer et al., 2003; Bayles \& Katerdahl, 2009; Weller et al., 2002). An individual experiencing susto may have symptoms of listlessness, anxiety, social withdrawal and restless sleep (Bayles \& Katerdahl, 2009).

Hispanic health paradox. Findings that Latino/as demonstrate a lower rate of pathology are not new nor unique to psychology. This phenomenon has been studied extensively in multiple health fields and has been titled the Hispanic or Latino health paradox, also referred to as the immigrant health paradox (Cho, Frisbie, Hummer \& Rogers, 2004; Ruiz, Hamann, Mehl, \& O’Connor, 2016). This paradox lies in the counterintuitive finding that despite Latino/as exhibiting higher rates of known risk factors for poor health including poverty, lower access to healthcare, and discrimination, they demonstrate better physical and mental health overall as a group compared to the non-Latino/a White population (Ruiz et al., 2016). A representative example of this being the finding that Latino/as have a greater life expectancy than the non-Latino/a White and non-Latino/a Black populations (CDC, 2015).

Interestingly, this paradox follows a linear path along acculturation in an unexpected direction; the more acculturated the individual, the more likely they are to show higher morbidity of mental and physical health conditions. Acculturation is measured by many factors including language fluency and engagement in the cultural practices of the host country (the country to which the individual immigrated). It is important, however, to disentangle a cumulative and general finding of higher morbidity to identify which specific physical and mental health conditions Latino/as experience at lower (or higher) rates. One such category of mental health disorders that appears to fall 
into the paradox is anxiety disorders. A recent study of Latino men provided support for the positive association between acculturation and incidence of disordered anxiety. Using logistic regression, the authors found that men who had lived in the United States longer than 11 years were significantly more likely $(\mathrm{OR}=.232)$ to meet criteria for generalized anxiety disorder than were men who had lived in the country for 5-10 years $(\mathrm{OR}=0.037$; Ai, Pappas, \& Simonsen, 2015).

Proxies of acculturation (e.g., birthplace, language fluency) have been found to be associated with anxiety in Latino/a adolescents such that being less acculturated to American culture predicted anxiety symptoms (Glover et al., 1999). However, this unidimensional view is challenged by findings that Latino/a adolescents less connected to Latino/a culture are also at higher risk for internalizing psychopathology when compared to Latino/a adolescents with a strong bicultural identity (Carvajal, Hanson, Romero, \& Coyle, 2002). Smokowski, Buchanan, and Bacallao (2009) used measures of both Latino/a and American identity in an attempt to capture a bicultural identity in a sample of 281 Latino/a adolescents and their parents. They found no significant association between adolescent American identity and any measured mental health outcome, including anxiety and depressive symptoms. However, they found parent involvement in U.S. culture was associated with lower rates of adolescent anxiety. Adolescent Latino/a identity was only significantly associated with lower rates of hopelessness, social problems, and aggression as well as higher rates of self-esteem. These findings support the importance of measuring acculturation not only from a bidimensional lens but also including parental acculturation. 
Various explanations for the Hispanic health paradox have been proposed and disputed. One such explanation is termed the healthy migrant hypothesis (Rubalcava, Teruel, Thomas, \& Goldman, 2008; Teruya \& Bazargan-Hejazi, 2013). The premise of this hypothesis is that immigrants display better health outcomes because of an inherent selection bias in that individuals who choose to immigrate represent a healthier subset of the population; in other words, those with poorer health are less likely to immigrate than their healthier counterparts. Though it is an intriguing explanation, the hypothesis has received weak support in studies including collective data from both home and host countries (Rubalcava et al., 2008). Another rationale for the Hispanic health paradox is provided by the salmon bias which states that the lower prevalence of disease is because immigrants relocate back to their home countries if they become unhealthy; however, this hypothesis has also been directly refuted (Bostean, 2013; Palloni \& Arias, 2004).

Considering the insufficient explanations provided by the healthy migrant and salmon bias hypotheses, researchers have turned to instead examining protective cultural factors in the Latino/a population. The positive association of acculturation with Latino/a mental health has led to a framework for the resilience exhibited in the Hispanic health paradox (Ruiz, Hamann, Mehl, \& O'Connor, 2016). The premise of the framework is that in the process of acculturation, there is a loss of a cultural trait or practice that is protective. Operating under this framework, following is a review of one such cultural characteristic or belief, fatalism, that could potentially be serving a protective role for anxiety in Latino/as. Fatalism is not unique to Latino/as, however, it has been found to be more prominent in this group as compared to others (Ross, Mirowsky, \& Cockerham, 1983). 


\section{FATALISM}

Fatalism is not a recently conceived term nor a new area of interest. Literature on fatalism dates back to the late 1800s in Émile Durkheim's book on suicide (Durkheim, 1897) in which he termed fatalistic suicide to describe suicide spurred by oppression and excessive social regulation. The modern use of term fatalism can be generally defined as the belief that outcomes are predetermined by fate, destiny, or other external forces (Ross et al., 1983). Empirically defining fatalism has proved to be an elusive venture. Beyond the broad definition of fatalism, some researchers have defined it as an acceptance of one's circumstances (Futa, Hsu, \& Hansen, 2001) while others define it as commensurate to pessimism and helplessness (Scheier \& Bridges, 1995). The disparity in the definition of fatalism has served as a barrier for subsequent work in this area. Establishing a foundation of fatalism research is made difficult when direct comparison of studies is not available due to variable definitions and measurement. Further, these differences in semantics come with attributions placed on the construct of fatalism and its effects. For example, if one study conceptualized fatalism as acceptance while another did so as helplessness, the measures used, the way the topic is communicated to the participants, the theoretical basis, the hypotheses, and the analysis of the data can all be affected. In addition to issues pertaining to defining fatalism generally, it is unclear if fatalism as part of a shared culture is best described as a belief, a value, or a trait factor. This question has gone largely ignored in the study of fatalism.

There is debate and confusion as to the connection between fatalism and spirituality. Fatalism has historically been viewed as a maladaptive form of adjustment yet has also conceptually and empirically been demonstrated to be positively related to 
spirituality (Cotton et al., 1999). Spirituality on the other hand has been supported as a source of support and can be a protective factor against a number of detrimental outcomes (Bernard et al., 2017; Paranjape \& Kaslow, 2010; Richards, Petrie, \& Chapman, 2016).

Fatalism has been studied in many fields of research, including public health, medical sciences, philosophy, social work, education, religious studies, and psychology. Consequently, fatalism has been studied in relation to a wide array of outcomes including physical and mental health outcomes, academics, rates of teenage pregnancy, and interpersonal styles such as assertiveness (e.g., Abraído-Lanza, Martins, Shelton, \& Flórez, 2015; Anastasia \& Bridges, 2015; Guzmán, Santiago-Rivera, \& Hasse, 2005; Rosales \& Calvo, 2017). The literature on fatalism is more prevalent in certain areas of study than others, though it would, theoretically, pervade throughout many aspects of an individual's life as well as have collective implications on a larger scale.

Measures of fatalism. Many independent measures of fatalism have been used over the course of fatalism research. There appears to be little overlap in measurement with many studies electing to either adapt existing self-report measures or create their own form of measurement unique to their study (Espinosa de los Monteros \& Gallo, 2011). In fact, a review of fatalism research found cumulatively 51 distinct measures (Esparza, 2015). Further, analysis of the content of these measures found the content varied greatly, likely dependent on the definition and positive or negative view of fatalism endorsed by the authors.

The Mini-MAC (Watson et al., 1994) is a 29-item self-report measure intended to assess an individual's adjustment to cancer, whether it be a new diagnosis or adjustment 
to the treatment process and the possibility of death. The Mini-MAC is a frequently used tool in the study of fatalism and health outcomes; however, is limited to fatalism in the context of cancer and is not able to be used in a broader study of fatalism. The Powe Fatalism Inventory (PFI; Powe, 1995) is another measure assessing fatalism in the context of cancer and is widely used in public health fatalism research. The PFI is a 15item self-report measure that was originally developed in a sample of African American women and has since been tested with and adapted for other groups. Shen, Condit, and Wright (2009) expanded upon the PFI by widening the scope by adapting it to more general health beliefs. However, with items such as "my health is a matter of luck" and "if someone is meant to have a serious disease, they will get that disease," this scale remains a measure of health beliefs and therefore is not useful outside of a health fatalism context.

There is a lack of a gold-standard measure for cultural fatalism, or fatalism not in the context of health beliefs and behaviors. Studies of cultural fatalism too often use study-specific measures or related, but not exact, subscales of other measures, such as measures of acculturation and locus of control (e.g., Anastasia \& Bridges, 2015; Greenwell \& Cosden, 2009; Joiner et al., 2001). This, again, makes comparison between studies a challenge. Abraído-Lanza and colleagues (2007) summarized the disjointed nature of the measurement of fatalism, noting that in addition to the previously stated issues, the established fatalism measures were created with and for non-Latino/a populations.

The measure that appears to be most widely used to study cultural fatalism in Latino/as is the fatalism subscale of the Multiphasic Assessment of Cultural Constructs 
(MACC; Cuéllar et al., 1995). The MACC, as the name indicates, is a self-report measure of cultural constructs, specifically developed for measurement in the Latino/a population. Along with the fatalism subscale, the measure includes items assessing familism, machismo, folk beliefs, and personalism. Eight true or false items make up the fatalism subscale. Items include, "people die when it is their time and there is not much that can be about it," "it is not always wise to plan too far ahead because many things turn out to be a matter of good and bad fortune anyway," and "it is more important to enjoy life now than to plan for the future." Other items make specific mention of control being in the hands of God and believing a doctor's diagnosis despite disagreeing. Collectively, these items appear to capture multiple definitions of fatalism including a facet of acceptance, importance of the present moment, and relinquished control. This could be seen as both a strength and weakness of the measure. On one hand, the comprehensive nature could be more inclusive of all that fatalism can encompass and prevent confirmation bias of the definition most arbitrarily favorable to the authors. On the other hand, the diversity in items may lack unity in that instead of combining to measure fatalism, they may be measuring three or four different beliefs or attitudes in close relation to fatalism and are possibly distinct subsets of fatalism. Supporting this hypothesis is the poor internal consistency data for the fatalism subscale ( $\alpha=0.63$; Cuéllar et al., 1995).

More recently, Esparza, Wiebe, and Quiñones (2015) developed a multidimensional scale of fatalism with simultaneous development in English and Spanish languages, using a Latino/a majority sample (86.2\% Latino/a). This self-report measure consists of thirty items rated on a five-point Likert scale ranging from 1 (“Strongly disagree”) to 5 ("Strongly agree”). The measure contains five subscales: 
divine control, luck, helplessness, internality, and fatalism. The multidimensional nature of this measure provides an added level of analysis, allowing for a simultaneous examination of the various constructs fatalism has been equated to; however, titling the measure as an assessment of fatalism when, in fact, fatalism is only one subscale is confusing and could lead to unintended use of the scale. The fatalism subscale contains six items and includes, "I have learned that what is going to happen will happen," "If something bad is going to happen to me, it will happen no matter what I do," and "Life is very unpredictable, and there is nothing one can do to change the future." Unlike the MACC, this measure parses out the role of God or religion from fatalism and places items such as "Whatever happens to me in my life, it is because that is the way God wanted it to happen" under the subscale of divine control. Model fit for this measure was good $(\mathrm{RMSEA}=0.04, \mathrm{CFI}=0.97, \mathrm{NFI}=0.94, \mathrm{GFI}=0.96, \mathrm{SRMR}=0.05)$ while the testretest reliability for the fatalism factor was less impressive but still acceptable $(r=0.71)$.

The current literature contains only cognitive measures of fatalism. Familism, unlike fatalism, has been measured in a variety of domains. Familism can be assessed by an individual's behavior, attitudes, and structure (i.e. proximity to family; ValdiviesoMora, Peet, Garnier-Villarreal, Salazar-Villanea, \& Johnson, 2016). It is unknown, however, if and how fatalism manifests outside of cognitions.

Public health research. The public health field has been the leader in fatalism research both generally and in research specific to Latino/as. Fatalism has been studied in relation to Latino/as experiences of cancer, diabetes, and stroke, among several other health conditions (e.g., Sharrief et al., 2017; Spurlock \& Cullins, 2006; Walker et al., 2012), particularly as a risk factor for poor health. Early studies of fatalism in relation to 
the health of Latino/as sought first to establish fatalism as a relevant construct. An early study by Hubbell, Chavez, Mishra, and Valdez (1996) used data from telephone surveys to determine differences between Latina and non-Latina White women on beliefs about breast cancer. They found a number of differences, including a finding that Latina women were significantly more likely to endorse fate as a reason for getting breast cancer.

The usual determinants, such as the type of cancer, cancer stage, and the age of the individual do not appear to be the source of observed differences in treatment of cancer for Latina women. With these factors controlled for, researchers have instead looked to cultural characteristics that may be playing an influential role in prevention and treatment efforts. As such, fatalism has been explored as a potential source that is particularly pertinent for Latino/as, given their tendency toward fatalistic beliefs (Neff \& Hoppe, 1993). Conceptually, researchers have connected fatalism as related to both preventative and treatment efforts due to the underlying, assumed premise of fatalism that action is futile in changing predetermined fate.

Substantial research in this area has found fatalistic beliefs to be a predictor of decreased participation in health-promoting or treatment-seeking behavior among Latino/as (see Espinosa de los Monteros \& Gallo, 2011 for a review). In addition to prevention and treatment behavior and practices, another area of cancer research in which fatalism is often studied is individuals' reactions to a cancer diagnosis, commonly referred to as psychological adjustment. Psychological adjustment in relation to a cancer diagnosis is a well-established research topic in the psycho-oncology literature. Through this work, five survival schemas have been identified. These schemas include anxious 
preoccupation, cognitive avoidance or denial, helplessness or hopelessness, fighting spirit, and fatalism (Moorey \& Greer, 1989). Naturally, some schemas are believed to be more adaptive and healthier than others. For example, fighting spirit has previously been found to be related to better survival rates than has helpless/hopeless or fatalistic responses (Greer, Morris, and Pettingale, 1979; Pettingale, Morris, Greer, \& Haybittle, 1985). From these findings, researchers have conceptualized fatalism as an individual's belief that because health outcomes are predetermined, any efforts to change health outcomes would be in vain which leads to inaction. There are, however, alternate perspectives with empirical support, such as a Cotton and colleagues (1999) study that found fatalism to be a positive predictor of quality of life in a sample of 142 women with breast cancer.

Mental health research. The study of fatalism and physical health outcomes, such as cancer, overlaps with the study of fatalism and mental health outcomes. For example, there is evidence that Latina women with higher levels of anxiety were more likely to attend breast cancer screenings (Cadet, Berrett-Abebe, \& Stewart, 2017) though this could vary based on the type of anxiety the individual experiences. Unlike general anxiety, feelings of embarrassment predicted a lower likelihood of participating in cancer screening (Magai, Consedine, Neugut, \& Hershman, 2007). It may be that fatalism is an important contributor to this process, but a comprehensive study of the relation of fatalism and anxiety has not yet been produced.

In psychological study, fatalism has been most commonly explored in relation to depression with a handful of studies also exploring suicidal ideation and trauma symptoms in Latino/as. The cumulative research has yielded mixed results, meaning 
fatalism has not clearly emerged as either entirely positive or negative across different outcomes. A couple of early studies of fatalism and mental health outcomes found evidence supporting fatalism as a correlate of higher levels of depression in two different age groups (Neff \& Hoppe, 1993; Roberts, Roberts, \& Chen, 2000). Neff and Hoppe (1993) conducted a large study investigating racial/ethnic differences in acculturation, depression, fatalism, and religiosity and the potentially different relationships across these variables dependent on race/ethnicity. Participants in their study were of MexicanAmerican, African-American, and non-Latino/a White ethnic background. Data for this study was collected from a sample of 1,789 adults and obtained as part of a larger study investigating differences in drinking habits across racial/ethnic groups. Being that this study was conducted in the early 1990s, with data having been collected in the late 1980s, most of the common measures for the constructs they assessed were not yet developed. With the exception of their use of the Center for Epidemiologic Studies Depression Scale (CES-D; Radloff, 1977), the authors created their own measures, which were unique to this study, for their variables of interest. Fatalism was measured using seven items drawn from two earlier studies (i.e., Chandler, 1979; Pearlin \& Schooler, 1978).

Results supported a higher endorsement of fatalism among Mexican Americans compared to non-Latino White individuals, but interestingly the difference only existed when comparing non-Latino White participants to Mexican Americans low in acculturation. This could potentially suggest that fatalism dissipates in the acculturation process. In analyzing fatalism in relation to depressive symptoms, results indicated those higher on fatalism endorsed significantly higher rates of depressive symptoms. Roberts, Roberts, and Chen (1998) conducted a similar study of fatalism but with a sample of 
middle school aged adolescents. They, too, found a significant positive association between fatalism and depressive symptoms. Further, in a later study, they also found fatalism was positively related to suicidal ideation in a sample of adolescents. The samples used for these studies, however, were ethnically diverse and contained a small number of Latino/a adolescents, precluding any conclusions about the effects of fatalism in a Latino/a cultural context (Roberts, Roberts, \& Chen, 2000).

More recently, Anastasia and Bridges (2015) conducted a study of fatalism in relation to depressive symptoms and health service utilization. Data were collected in both English and Spanish from 83 Latino/a adults who completed self-report questionnaires including the Brief Symptom Inventory (BSI-18; Derogatis, 2001), the fatalism subscale of the Multiphasic Assessment of Cultural Constructs-Short Form (MACC-SF; Cuéllar et al., 1995), and the Service Utilization Interview (SUI; Bridges, 2008). Results of this study indicated fatalism was associated with increased depressive symptoms as well as decreased service utilization in that respondents high in fatalism had attended medical services significantly less in the past year.

In a study comparing adolescent Mexican-American girls with other adolescent girls, Joiner, Perez, Water, Berenson, and Marquina (2001) found that the MexicanAmerican girls demonstrated a higher rate of depressive symptoms, but this was explained by their higher reports of pessimism and not fatalism. In the same vein, PiñaWatson and Abraído-Lanza (2017) made the argument that fatalism is not simply either detrimental or beneficial to psychological health. Instead, they propose, it is the interaction of fatalistic beliefs with negative attributions that is detrimental to mental health. Of note, in an attempt to parse pessimism from fatalism, the authors adapted an 
established measure of fatalism (Shen et al., 2009). This measure contains 20 items which Piña-Watson and Abraído-Lanza (2017) separated into three categories, predetermination, luck, and pessimism, which enabled them to compare between the categories as opposed to only having a single construct of fatalism. In addition, they changed the language to be more general as opposed to specific to health beliefs as well as to accommodate their adolescent sample. Examples of items include, "if something bad is meant to happen to me, it will happen," "my life events are a matter of luck," and "my life is determined by something greater than myself."

Aside from outcomes related to depression and suicide, only a single study on trauma symptoms that sought to examine fatalism and clinical mental health outcomes exists. Greenwell and Cosden (2013) explored the potential of fatalism as a predictor of trauma symptoms and peritraumatic dissociation among a sample of 112 low-income Latino/as attending an outpatient mental health clinic. All participants were survivors of domestic violence. In addition to measures for fatalism and trauma symptoms, the study included an acculturation variable to investigate if levels of fatalism were dependent on acculturation. Fatalism was measured using the 8-item fatalism subscale of the MACCSF (Cuéllar et al., 1995); however, preliminary analysis revealed low internal reliability for this measure in their sample. The authors then chose to, instead, use the five items with the best correlation coefficients in their analyses as opposed to the full subscale. Another modification made from the original was the response options provided. This study used an 8-point Likert scale instead of the true/false response options on which the MACC-SF was originally developed. Results indicated fatalism found was not significantly associated to trauma symptom severity (Greenwell \& Cosden, 2013). 
Further, acculturation was not predictive of peritraumatic dissociation. The authors cite no significant difference in fatalism along acculturation level, however, they measured acculturation in a non-standardized way using proxy variables such as language preference. This study's modifications in the way fatalism was measured makes it complicated to make direct comparisons to other studies. Noticeably absent from the literature is an investigation of fatalism in relation to anxiety.

Culture and fatalism. Many problems exist in the study of fatalism in relation to health (physical and mental). Though fatalism and related health outcomes have been studied extensively in the Latino/a population, much of the research appears to view fatalism from a non-Latino/a ethnocentric perspective. The normality of fatalism likely varies by culture, meaning while in one culture it may be a maladaptive deviation from the norm, it could be a well-integrated norm in another culture. To this end, Diaz, Blanco, Bajo, and Stavraki (2015) distinguished between fatalism as experienced in an individualistic culture (Spain) and a collectivistic culture (Colombian). This study was conducted in the context of creating a self-report measure of fatalism that has been validated with two similar but distinct cultural groups. Results indicated fatalism was associated with lower levels of subjective and social well-being in the individualistic sample, whereas in the collectivistic group, fatalism remained negatively related to social well-being but was positively related to subjective well-being.

The sociocultural context Latino/as live in is a powerful variable and is too often ignored. To this end, it is imperative to understand the factors that create, foster, and maintain fatalism. There is a lack of a theoretical framework from which to base the associations between fatalism and health outcomes (Abraído-Lanza et al., 2007). As such, 
individual studies have defined and conceptualized fatalism in different manners and interpreted a variety of sociocultural factors as contributors to fatalism. Latino/as, as a whole and in the United States particularly, face significant stressors of discrimination, poverty, and subpar access to resources, such as education and health care. These factors constitute a different daily reality than that of the typical reference group used in scientific research which is skewed toward the non-Latino/a White population. The point of view, therefore, that certain aspects of life are inevitable may then feel more true for the Latino/a population as opposed to being a negatively biased or misrepresented outlook on the power of the individual. In which case, perhaps fatalism is not a helpless resignation but instead an appropriate or adaptive recognition of barriers.

The effect of fatalism on behavior is not as clear as promulgated. Meta-analyses and literature reviews of research relating fatalism to decreased cancer screenings have collectively deemed this relationship inconclusive. Espinosa de los Santos and Gallo (2011) looked specifically at fatalism and cancer screening while controlling for age, SES, acculturation, and health care access. They found that while the slight majority of the studies they reviewed found a significantly negative relationship $(n=7)$, the findings were fairly even and an almost equal number of studies did not find a significant relationship $(n=6)$.

Collectively, research on fatalism has largely described it as a risk factor for psychopathology. However, there have been intermittent calls for a re-assessment of the way fatalism is conceptualized. Parker and Kleiner (1966) presented an early viewpoint of fatalism as having the capability to provide positive influence. Their work proposed that fatalism could be an adaptive response to uncontrollable life events experienced by 
ethnic minorities. For example, in a qualitative analysis of fatalism, Benavides, Bonazzo, and Torres (2006) captured a personal account of fatalism that can be seen as containing adaptive qualities. They quote an individual participating in their study as saying they acknowledge their risk for developing a serious health condition but instead of expressing fear or concern the subject says, "I might as well enjoy myself while I am alive" (p. 87, Benavides, Bonazzo, \& Torres, 2006). In this statement lies the crux of the argument that while it may be true that fatalism has a detrimental effect on certain aspects of health, the framing of problems as not a cause for worry could make it protective for psychopathology generally and anxiety disorders specifically. Meaning, if an individual has the idea that future events are predetermined, it can be hypothesized they are engaging in less "what if" thinking characteristic of worry. Further, this could lead to an increased practice of being in the present moment and enjoying life as it is happening, as was alluded to by the Benavides, Bonazzo, and Torres (2006) participant.

\section{FATALISM, ANXIETY, AND WORRY}

The literature on models of anxiety, and specifically worry, is extensive.

Particularly of interest here is worry, the cardinal feature of generalized anxiety disorder. Worry models can be categorized into cognitive models and emotion-focused models. Of these, cognitive models have the greater theoretical overlap with fatalism. A prominent cognitive worry model is the Cognitive Avoidance model (Borkovec, 1994; Borkovec, Alcaine, \& Behar, 2004) which, generally, describes worry as a form of cognitive avoidance in that worry abstracts the feared outcomes and avoids the heightened arousal associated with direct representations such as vivid images. Another cognitive model of worry is the Intolerance of Uncertainty model (Dugas, Gagnon, Ladouceur, \& Freeston, 
1998). Intolerance of uncertainty is described in depth in the following section. Lastly, the Metacognitive model of worry (Wells, 1999) describes an individual's beliefs about worry and how these beliefs can reinforce worry by either finding worry useful and necessary or by believing worry to be frightening and unmanageable.

The applicability of models of worry to the Latino/a population has not been studied. Developing an understanding of the process of anxiety and worry in the Latino/a culture from a theoretical framework would be an important contribution to the literature on culture and psychopathology. Given the collective research on the Hispanic health paradox, anxiety prevalence in Latino/as, and fatalism, it can be hypothesized that the cultural aspect of fatalism plays a role in both the content and the process of worry. Importantly, it is not suggested that fatalism can account for all aspects of worry. It is plausible, though, to hypothesize that fatalism alters specific theoretical pieces of worry in a way that is influential and could potentially account for differences seen in prevalence rates. Guided by extant models, key cognitive constructs associated with anxiety and worry are conceptually connected to fatalism in a manner proposing fatalism as a potential protective factor for anxiety and worry. The relevant constructs and themes across cognitive models of worry include intolerance of uncertainty, control, coping, and mindfulness and acceptance.

Intolerance of uncertainty. An alternative perspective, in contrast to previous arguments that fatalism leads to feelings of helplessness, is a theoretical connection between fatalism and intolerance, or rather tolerance, of uncertainty. Intolerance of uncertainty (IU) has been identified as an important factor implicated in excessive worry. IU is defined as "a dispositional characteristic that results from a set of negative beliefs 
about uncertainty and its implications" (Dugas \& Robichaud, 2007, p. 24). There are a number of key aspects of IU, several of which can be hypothesized as relating conceptually to fatalism. In the development of the Intolerance of Uncertainty Scale (IUS; Buhr \& Dugas 2002), a four-factor structure was found. The four factors are: uncertainty leads to the inability to act, uncertainty is stressful and upsetting, being uncertain about the future is unfair, and unexpected events are negative and should be avoided.

Importantly, in the development of the IUS, and hence the development of a fourfactor structure, the majority sample was comprised of non-Latino/a White participants. This leaves the question of the applicability of this model across other ethnic groups. To this end, Norton (2005) investigated the validity of the IUS across four racial/ethnic groups, African Americans, Southeast Asians, Latino/as, and non-Latino/a White individuals. For Latino/as, results suggested a six-factor solution was most appropriate. The six-factor structure, however, was stated to be "poorly interpretable" therefore the factors were not labeled leaving in question what aspects of IU are important for Latino/as.

Utilizing the four-factor model, it seems that fatalism could have an influential and protective role in three of the four. First, the premises that uncertainty is stressful and upsetting and that uncertainty is unfair are challenged by fatalism. Fatalism views uncertainty as a fact of life, and with fatalism as a cultural norm, it is a commonplace belief not a rarely encountered stressful and upsetting experience. Relatedly, the belief that unexpected events are negative and should be avoided is in conflict with fatalism. From the view of fatalism, unexpected events are a certainty because the individual 
cannot accurately predict what fate has in stored and therefore cannot be avoided. It is proposed fatalism may be negatively related to IU because fatalism recognizes and accepts uncertainty. In other words, instead of avoiding the possibility of uncertainty by worrying, individuals high in fatalism accept there is uncertainty and allow the future to happen as it comes.

Control. In addition to IU, fatalism is of particular interest in relation to anxiety because of a shared theme of perceptions of control. There is evidence that Latino/as may have a different relationship with control as early as in childhood development. A prime example is the differential outcomes Latino/a youth and non-Latino/a White youth experience as a result of controlling parenting. For non-Latino White children, the research suggests controlling parenting is associated with higher rates of anxiety (Muris, 2006; Wood, McLeod, Sigman, Hwang, \& Chu, 2003). On the other hand, it appears this connection between a controlling parenting style and anxiety does not exist for Latino/a children (Creveling, Varela, Weems, \& Corey, 2010; Luis, Varela, \& Moore, 2008; Varela, Sanchez-Sosa, Biggs, \& Luis 2009). This difference in norms may arise from an adaptive perspective in that it is an attempt from Latino/a parents to safeguard their children from engaging in risky behaviors or falling victim to the illicit activities of others which ethnic minority children, in general, are more likely to encounter. As related to fatalism, it may be that Latino/as are more accustomed to having less control, compared to non-Latino/a White individuals. Therefore, it may be that a lack of control over certain aspects of the future is not a new nor distressing concept to Latino/a adults.

The current literature frequently equates fatalism to helplessness because of the interpretation that fatalism necessarily indicates the individual believes there is nothing 
they can do to change the future making putting forth an effort toward change futile. Indeed, a perceived lack of control has been associated with higher levels of anxiety (Barlow, 2002). However, the perspective that marries fatalism to helplessness, therefore indicating a risk for anxiety, accounts for only direct, or primary, control. Primary control refers to having direct power to change outcomes. However, primary control is not the only type of control. In addition to primary control is secondary control. Secondary control is a form of mastery in which an individual does not have control over their environment but does have the power to modify themselves and accept circumstances as they are (Helzer \& Jayawickreme, 2015; Weisz, Rothbaum, \& Blackburn, 1984). In this sense, an individual high in fatalism could still maintain a high level of secondary control and potentially, thereby, not fall at risk for anxiety as they would if they felt no sense of control at all.

A second concept of control, primarily discussed in social psychology, is harmony control. Harmony control is an indirect means of control considering interdependence and is particularly relevant when studying non-Western European populations. Individuals with a sense of harmony control perceive control as not belonging to the individual but to the larger group or a higher power (Morling \& Fiske, 1999). While a lack of individual control may feel unsettling to some, this larger concept of harmony control may allow for comfort with uncertain outcomes by assigning control to a trusted entity, such as fate. This may especially be the case when harmony control is a culturally-propagated and normative belief.

Acceptance, mindfulness, and coping. Fatalism can also be hypothesized to be related to acceptance through conceptual links of the shared feature of low control, 
whether relinquished or perceived as unattainable. As opposed to a resigned helpless state, fatalism may instead present as an acceptance of circumstances outside of one's control. In discussing the potential explanations for the Hispanic health paradox, particularly in the case of immigrants, Alegría and colleagues (2008) suggested the relative deprivation experienced by immigrants in their home countries may lead to "decrease[d] likelihood of demoralization and increase[d] resignation to negative outcomes, resulting in lower risk of depression and anxiety" (Alegría et al., 2008, p. 366).

The lack of preoccupation with the future and predicting outcomes aspect of fatalism can be hypothesized to relate to present-moment and acceptance aspects of mindfulness as studied in Western psychology. This hypothesis is consistent with research on time orientation in the Latino/a population which states that in the Latino/a culture, the focus is on the present moment and is less future-oriented (Marin \& VanOss Marin, 1991). In the same vein, it appears that Latino/as tend to focus more on day-to-day survival rather than planning for the future. It could be this arose from an adverse environment requiring that level of present-moment attention which then led to a cultural normativity of present-focused attention. Fatalism could potentially be a form of coping with events outside of an individual's control, which may be more commonplace for ethnic minority individuals, such as Latino/as (Parker \& Kleiner, 1966).

\section{THE CURRENT STUDY}

The present study aims to analyze the relevance of fatalism in relation to anxiety and worry as it is yet only a conceptual link and does not appear in previous literature as a tested relationship. This work is an important step in developing a theoretical model of anxiety and worry in Latino/as. This could include adding fatalism to an established 
model of worry, such as the IU model (Dugas et al., 1998), based on associations found between fatalism and constructs within the model. It may also take the form of a model with fatalism as the focus, or common factor, and varied mental health outcomes that explains where it belongs in relation to these outcomes. These models could then be tested and lead to subsequent studies. For example, testing a model including fatalism, anxiety, and depression, given the studies finding fatalism is predictive of depressive symptoms and the shared aspect of negative affect in anxiety and depression. Again, drawing from the literature on familism could prove to be useful. For example, Hernandez and Bamaca-Colbert (2016) developed a behavioral process model of familism in relation to the psychological adjustment of youth in which they identified the mechanisms at work. One such model worth testing would be if the existence of different forms of control, such as secondary control, moderates the relation between fatalism and anxiety or worry in that secondary control may serve as a buffer.

The study of fatalism and its association with anxiety and worry may be particularly relevant for children and adolescents considering the importance of identity development in this stage of life and the influence acculturation may have on how a child or adolescent chooses to identify. Considering fatalism as a potentially protective factor, it then becomes very important to encourage retainment of fatalistic beliefs when decisions are being made as to which culture an individual chooses to identify with.

\section{Study Aims and Hypotheses}

Aim 1. Examine the cultural relevance of fatalism

Hypothesis 1. Identification with the Latino/a culture and, separately, with American culture will predict fatalism in opposing manners, such that higher Latino/a 
identification will be associated with being more fatalistic and higher American identification will be associated with being less fatalistic.

Aim 2. Test the conceptual connection between fatalism and anxiety symptoms.

Hypothesis 2. Fatalism will predict the adolescent's endorsement of anxiety symptoms, such that higher fatalism will be associated with lower levels of anxiety and worry.

Aim 3. Examine the role of fatalism in intolerance of uncertainty.

Hypothesis 3. Fatalism will predict intolerance of uncertainty, such that higher levels of fatalism will be associated with lower levels of intolerance of uncertainty.

Aim 4. Explore the role of control constructs as they relate to the association between fatalism and anxiety symptoms.

Hypothesis $4 a$. Fatalism will predict primary and secondary control in opposite directions, such that higher fatalism will correspond to lower primary control and higher secondary control.

Hypothesis $4 \boldsymbol{b}$. Secondary control will moderate the relation between fatalism and anxiety symptoms, such that higher fatalism will predict stronger secondary control, which will predict lower levels of anxiety and worry. 


\section{METHOD}

\section{Participants}

Forty-seven adolescents of Latino/a ethnic background were recruited from the University of Louisville Pediatrics Clinic over a period of 9 months, spanning from April to December 2019. The adolescent participants were ages 12 to 17 years old at the time of participation. Eligibility was limited to adolescents fluent in the English language and with no known developmental disabilities or delays.

\section{Recruitment}

Adolescents were recruited from the University of Louisville Pediatrics Clinic in Louisville, Kentucky. Patients who presented for a routine well child visit with a pediatrician in the clinic, and fit eligibility criteria, were asked to participate in the brief study. Flyers describing the study were posted in the clinic as well as distributed to clinic personnel including registration staff, medical assistants, nursing staff, resident physicians, and attending physicians. Flyers in both English and Spanish contained a brief description of the study, study personnel contact information, and a link and QR code to access the study.

\section{Procedure}

Parents of eligible adolescents were invited to have their adolescent participate in the study either prior to, during, or at the conclusion of their scheduled visit with the pediatrician. All participants were provided with the option to complete the study on a 
tablet made available to them or on their personal cell phones. The consent and assent process was handled in two ways, in accordance with IRB approval. Most participants $(\mathrm{N}=41,87.2 \%)$ were presented with consent and assent forms in person and participants and their parent/guardian provided written signatures. The remaining participants $(\mathrm{N}=6$, $12.8 \%$ ) were directed to an electronic version of these forms and provided consent by clicking "submit" at the bottom of the page. This shift in procedure was done in an effort to broaden the reach of recruitment by eliminating the need of the study coordinator to be physically present. Participants were then directed to the study questionnaires and narrative task. Study questionnaires, the narrative task, and collected data were managed by REDCap, a HIPAA compliant research tool that allows the participant's data to be linked to a study identification number for confidentiality. The length of time to complete the study in its entirety was approximately 30 minutes.

\section{Measures}

\section{Anxiety measures.}

Beck Anxiety Inventory-Youth (BAI-Y). The BAI-Y is an adaptation of the Beck Anxiety Inventory (BAI; Beck et al., 2005) designed for use with children and adolescents ages 7-18. The BAI-Y is a 20-item self-report scale measuring children's anxious beliefs (e.g., "I am afraid that I might get sick"), cognitions (e.g., "I worry I might go crazy"), and physiological responses (e.g., "My hands shake"). Each item asks the child to respond with whether they experienced the statement "never" "sometimes" "often" or "always" in the past two weeks. The BAI-Y has demonstrated convergent validity with other measures of anxiety in children, such as the Revised Children's Manifest Anxiety Scale (RCMAS; Reynolds \& Richmond, 1997), as well as high test- 
retest reliability $(r=0.64-0.88)$. Although the reliability of the BAI-Y has not been tested with Latino/a adolescents, multiple psychometric studies have shown the adult version is appropriate for use with Latino/as (Hirai, Stanley, \& Novy 2006; Novy, Stanley, Averill, \& Daza, 2001).

Penn State Worry Questionnaire-Child Version (PSWQ-C). The PSWQ-C is an adaptation of the Penn State Worry Questionnaire (PSWQ; Chorpita, Tracey, Brown, Collica, \& Barlow, 1997) designed for use with children and adolescents ages 7-17. The PSWQ-C is a 14-item self-report scale measuring worry (e.g., "My worries really bother me," "Many things make me worry"). Each item asks the child to respond with whether a description is "never true," "sometimes true," "most times true," or "always true." The PSWQ-C has demonstrated convergent validity with other child worry measures, such as the RCMAS Worry/Oversensitivity subscale, and high test-retest reliability $(r=.92$; Chorpita et al., 1997). The possible range of scores on this measure is 0 to 42 . Higher scores on this scale indicate higher levels of worry. As is the case for the BAI-Y, psychometrics studies of the PSWQ-C with Latino/a adolescents are not available in the extant literature; however, such studies have been conducted with Latino/a adults and found the PSWQ was appropriate for use with Latino/a adults (Hirai, Stanley, \& Novy 2006; Novy, Stanley, Averill, \& Daza, 2001).

\section{Fatalism measures.}

Modified Story Completion Task. In addition to a self-report questionnaire of fatalism, a more applied measure could corroborate the endorsements of fatalism. The present study uses a task in the form of narratives to present individuals in ambiguous situations (e.g., "Marco went to the doctor for a regular check-up. He was sent to do X- 
rays and told he will get the results in two weeks"). The participant was then asked how they believe the rest of the story continues. Ten scenarios were developed for this study (Appendix A). Prompts are purposefully future-oriented to allow for relevance of fatalism and include themes used in similar tasks in the literature (e.g., weather, social encounters, academic outcomes; Berna, Lang, Goodwin, \& Holmes, 2011; Chorpita, Albano, \& Barlow, 1996; Waite, Codd, \& Creswell, 2015). Next, participants were asked to rate how much they agree with a statement intended to assess their level of fatalism (e.g., "What happens to Marco is determined by fate") on a scale of 1 ("strongly disagree") to 5 ("strongly agree"). Fatalism items are modified statements derived from the Multiphasic Assessment of Cultural Constructs- Short Form (MACC-SF; Cuéllar et al., 1995) and the Multidimensional Fatalism Scale for General Life Events (MFS-G; Piña-Watson \& Abraído-Lanza, 2017). Participants were asked to rate how worried they believe the subject is on a scale of 1 ("not at all") to 5 ("very, very worried").

Revised Multidimensional Fatalism Scale (MFS). This scale is a 30-item selfreport questionnaire designed to measure fatalism and related constructs. Each item is rated on a five-point Likert scale ranging from 1 ("Strongly disagree") to 5 ("Strongly agree"). The multidimensional fatalism scale contains five subscales: divine control, luck, helplessness, internality, and fatalism. The fatalism scale was used for analyses in the present study. The factor structure of the measure in both English and Spanish is supported, as is the test-retest reliability and the convergent and discriminant validities (Esparza, Wiebe, \& Quiñones, 2015). This measure was developed with a group of undergraduate students $\left(\mathrm{M}_{\mathrm{age}}=20.34\right)$ and has not been tested with adolescents. However, with a slight modification of one item to say, "How successful people are in their job is 
related to how lucky they are" to "How successful people are in school is related to how lucky they are," the language of the measure appears to be appropriate for use with adolescents.

\section{Intolerance of Uncertainty measure.}

The Intolerance of Uncertainty Scale for Children (IUSC). The IUSC is an adaptation of the Intolerance of Uncertainty Scale for use with children and adolescents ages 7-17 (IUS; Comer et al., 2009). The IUSC is a 27-item self-report scale measuring negative emotional, cognitive and behavioral reactions to uncertain situations and events (e.g., "Not knowing what will happen in the future makes life hard, "Surprise events upset me greatly.") Each item asks the child to indicate how well a statement describes them on a scale from one to 5, where one is "Not at all," three is "Somewhat," and five is "Very much." The IUSC has demonstrated convergent validity with measures of anxiety and worry including the Multidimensional Anxiety Scale for Children (MASC) and the PSWQ-C. The IUSC has also demonstrated acceptable utility in distinguishing youth with anxiety disorders from healthy controls, with a cut score of 50 to 54 correctly classifying $80 \%$ of youth with anxiety disorders (Comer et al., 2009). Youth with GAD tend to report higher levels of IU $(M=66.61)$ than youth with other anxiety disorders (M=59.49; Read et al., 2013). The possible range of scores on this measure 27 to 135 with higher scores indicating higher levels of IU (Comer et al., 2009). A psychometric analysis of cross-cultural validity of the IUS found uniformity across groups, including Latino/as, providing strong evidence of its appropriateness of its use with this population (Norton, 2005). The Norton (2005) study was conducted with a sample of adults, 
specifically undergraduate students. To our knowledge, no cross-cultural psychometric analysis has been conducted for the IUSC.

\section{Control Measures.}

Perceived Control Scale for Children (PCSC). The Perceived Control Scale for Children is a 24-item self-report measure designed to assess beliefs about the individual's control over behavioral, social, and academic outcomes (Weisz, Southam-Gerow, \& Sweeney, 1998). This measure was developed with children and adolescents aged 8-17. This scale includes half reverse-coded items (e.g., "I cannot get other kids to like me no matter how hard I try"). Items are rated on a four-point Likert scale ranging from 1 ("Very false") to 4 ("Very true). This measure has demonstrated appropriate internal consistency and test-retest reliability (Weisz et al., 2001). Psychometric analysis of the PCSC for Latino/a adolescents does not exist in the literature; however, a study with a $40 \%$ Hispanic youth sample found the PCSC to have an overall alpha of .89. The present study found an alpha of .84, supporting previous findings and indicates good reliability for use of the PCSC with Latino/a adolescents.

Secondary Control Scale for Children (SCSC). The SCSC is a 20-item selfreport measure that assesses children's beliefs about their ability to control their psychological responses to outcomes (e.g., "I can usually find something to like, even in a bad situation; Weisz, Francis, \& Bearman, 2010). It was originally developed in a study with children ages 11-14, in a sample with a large Latino/a representation (40\%). With no reliability data available for older adolescents, comparison analyses were conducted between younger (11-14) and older (15-17) adolescents for the current study to ensure there were no systematic differences before combining the two age groups. Each item is 
rated using a four-point Likert scale ranging from 1 ("Very false") to 4 ("Very true").

This measure has demonstrated adequate internal consistency, test-retest reliability, and convergent and discriminant validity (Weisz et al., 2010).

\section{Acculturation Measure.}

\section{Bicultural Involvement Questionnaire (BIQ)-Adolescent and Guardian Report.}

The BIQ is a measure of acculturation using 33 items to assess level of comfort in the American culture (Americanism subscale) and the Hispanic culture (Hispanicism subscale) independent of each other. It was developed for use with Latino/a adolescents ages 11-18 and their guardians (Szapocznik, Kurtines, \& Fernandez, 1980). Each item is rated using a five-point Likert scale ranging from 1 ("Not at all") to 5 ("Very much"). This measure was found to have good internal consistency and test-retest reliability (Szapocznik et al., 1980). A later psychometric study of the BIQ suggests the best use of this measure in analyses is a four-factor scoring procedure with separate subscales for Spanish language, Hispanic activities, English language, and American activities (Guo, Suárez-Morales, Schwartz, \& Szapocznik, 2009). For this study, we collected data from both the adolescent and their guardian, with each filling out the questionnaire. As suggested by Szapocznik and colleagues (1980) and validated by Guo and colleagues (2009), the measure is slightly different for each group with the adolescent measure excluding two work-related items and the guardian measure excluding two school-related items. The guardian acculturation report was used as a control, based on previous research highlighting its influence (Smokowski et al., 2009). 


\section{RESULTS}

\section{Preliminary Examination of Measures}

Two participants did not complete the primary and secondary control measures due to ending their participation early. Additionally, fourteen parents did not complete the measure of parent acculturation. Little's Missing Completely at Random (MCAR; Little, 1988) test was nonsignificant, $p=1.0$, indicating that data were MCAR. Missing data were therefore addressed using pairwise deletion (Gelman \& Hill, 2006).

\section{Preliminary Analysis}

Descriptive statistics. Demographic characteristics for the current sample are displayed in Table 1. Means and standard deviations of each measure for the current sample are displayed in Table 2.

Table 1

Individual and Family Characteristics of the Sample

\begin{tabular}{|c|c|c|c|c|}
\hline Characteristic & $\mathrm{N}$ & $\%$ & $\mathrm{M}$ & $\mathrm{SD}$ \\
\hline \multicolumn{5}{|l|}{ Gender } \\
\hline Female & 28 & $59.6 \%$ & & \\
\hline Male & 18 & $38.3 \%$ & & \\
\hline Other & 1 & $2.1 \%$ & & \\
\hline \multicolumn{3}{|l|}{ Age } & 14.36 & 1.73 \\
\hline 12 & 9 & $19.1 \%$ & & \\
\hline 13 & 8 & $17.0 \%$ & & \\
\hline 14 & 10 & $21.3 \%$ & & \\
\hline 15 & 2 & $4.3 \%$ & & \\
\hline 16 & 13 & $27.7 \%$ & & \\
\hline 17 & 5 & $10.6 \%$ & & \\
\hline \multicolumn{3}{|l|}{ Grade } & 8.81 & 1.83 \\
\hline 6 & 9 & $19.1 \%$ & & \\
\hline
\end{tabular}




\begin{tabular}{llll}
\hline Characteristic & $\mathrm{N}$ & $\%$ & $\mathrm{M}$ \\
\hline 7 & 3 & $6.4 \%$ \\
8 & 6 & $12.8 \%$ & \\
9 & 10 & $21.3 \%$ \\
10 & 10 & $21.3 \%$ \\
11 & 7 & $14.9 \%$ & \\
12 & 2 & $4.3 \%$ & \\
Nationality & & & \\
Mexican & 22 & $46.7 \%$ \\
Cuban & 11 & $23.4 \%$ \\
American & 7 & $14.9 \%$ \\
Guatemalan & 2 & $4.3 \%$ \\
Colombian & 2 & $4.3 \%$ \\
Other & 3 & $6.3 \%$ \\
Child Immigrated & & \\
Yes & 11 & $23.4 \%$ \\
No & 36 & $76.6 \%$ \\
Parent Immigrated & & \\
Yes & 41 & $87.2 \%$ \\
No & 6 & $12.8 \%$ \\
Religion & & \\
Catholic & 29 & $61.7 \%$ \\
Atheist/Agnostic & 6 & $12.8 \%$ \\
Other & 10 & $21.4 \%$ \\
Decline to state & 2 & $4.3 \%$ \\
Annual Income & & \\
<20,000 & 18 & $41.9 \%$ \\
\$20,000-\$49,999 & 11 & $25.5 \%$ \\
\$50,000+ & 4 & $9.4 \%$ \\
Decline to state & 10 & $23.3 \%$ \\
\hline
\end{tabular}

Note. Annual income indicates household income. $\mathrm{N}=$ number of individuals; \%= percentage of individuals; $\mathrm{M}=$ mean; $\mathrm{SD}=$ standard deviation.

Table 2

Characteristics of Measured Variables

\begin{tabular}{llllll}
\hline Measure & M & SD & Min. & Max. & Com. Score \\
\hline BIQ-Self & & & & & \\
Hispanicism & 43.02 & 8.78 & 24 & 55 & 52.8 \\
Americanism & 44.26 & 7.52 & 19 & 55 & 47.4 \\
BIQ-Guardian & & & & & \\
Hispanicism & 47.76 & 7.80 & 29 & 64 & 51.84 \\
Americanism & 34.12 & 12.29 & 13 & 55 & 34.56 \\
BAI-Y & 17.26 & 12.26 & 2 & 53 & 18.5 \\
\hline
\end{tabular}




\begin{tabular}{llllll}
\hline Measure & M & SD & Min. & Max. & Com. Score \\
\hline PSWQ-C & 18.72 & 10.96 & 1 & 41 & 12.96 \\
IUSC & 60.43 & 23.52 & 30 & 119 & 52.81 \\
MFS-F & 18.94 & 4.57 & 6 & 30 & 17.76 \\
PCSC & 52.38 & 10.00 & 29 & 69 & 59.78 \\
SCSC & 38.25 & 10.48 & 18 & 59 & 40.32 \\
\hline
\end{tabular}

Note. $\mathrm{M}=$ mean; $\mathrm{SD}=$ standard deviation; Min. $=$ minimum score on the measure; Max.= maximum score on the measure; Com. Score= comparison score based on previous studies; $\mathrm{BIQ}=$ Bicultural Involvement Questionnaire; BAI-Y= Beck Anxiety Inventory for Youth; PSWQ-C = Penn State Worry Questionnaire for Children; IUSC= Intolerance of Uncertainty Questionnaire- Child; MFS-F= Multidimensional Fatalism Scale- Fatalism; PCSC = Primary Control Scale for Children; SCSC = Secondary Control Scale for Children.

The BIQ self-report demonstrated good reliability overall in the current sample (Cronbach's $\alpha=.82$ ). The Hispanicism (Cronbach's $\alpha=.88$ ) and Americanism subscales (Cronbach's $\alpha=.86$ ) also demonstrated good reliability. The present sample reported scores of slightly lower than previous studies of bicultural involvement in Americanism $(M=47.4)$ and much lower in Hispanicism ( $M=52.8$; Martinez, Schwartz, Their, \& McClure, 2018). One likely explanation is that in the comparison study, inclusion criteria required the youth be foreign-born whereas the present study did not control for immigration status and, in fact, the sample was majority U.S.-born.

The BIQ guardian report demonstrated excellent reliability overall (Cronbach's $\alpha$ $=.90)$ as did the Americanism subscale (Cronbach's $\alpha=.95)$ and the Hispanicism subscale (Cronbach's $\alpha=.87$ ) demonstrated good reliability. The present sample reported scores comparable in Americanism $(M=34.56)$ and Hispanicism $(M=51.84$; Chartier et al., 2015) to those of previous studies using a Latino/a American adult sample.

The BAI-Y demonstrated excellent reliability (Cronbach's $\alpha=.95$ ). The present sample reported scores comparable to past studies ( $M=18.5$; Steer, Kumar, Beck, \& Beck, 2001). 
The PSWQ-C demonstrated excellent reliability (Cronbach's $\alpha=.94$ ). The present sample reported scores higher than past studies of youth without an anxiety disorder diagnosis $(M=12.96)$ and lower than studies of youth with an anxiety disorder diagnosis ( $M=24.09$; Pestle, Chorpita, \& Schiffman, 2008). This is to be expected given the present study did not assess for anxiety disorder diagnosis and is likely a mix of a clinical and non-clinical participants.

The IUSC demonstrated excellent reliability (Cronbach's $\alpha=.96$ ). The present sample reported scores higher than past studies of youth without an anxiety disorder diagnosis ( $M=52.81$; Comer et al., 2009) and lower than studies of youth with an anxiety disorder diagnosis ( $M=64.97$; Cowie, Clementi, \& Alfano, 2016). Similar to the PSWQ$\mathrm{C}$, this is to be expected given the present study did not assess for anxiety disorder diagnosis and is likely a mix of a clinical and non-clinical participants.

The MFS demonstrated excellent reliability overall (Cronbach's $\alpha=.90$ ) whereas the fatalism subscale of the MFS demonstrated acceptable reliability (Cronbach's $\alpha=$ .75). The present sample reported scores comparable to a past study of adolescents in Mexico ( $M=17.76$; Maercker, Ben-Ezra, Esparza, \& Augsburger, 2019) and higher than a study of adolescents in Spain ( $M=12.72$; Navarro, Yubero, \& Larrañaga, 2018), though geographical, cultural differences may explain this gap in scores.

The PCSC demonstrated good reliability (Cronbach's $\alpha=.84$ ). The present sample reported scores significantly lower than a previous investigation of perceived control in children and adolescents $(M=79.46$; Magaro \& Weisz, 2006) and similar to a second study ( $M=59.78$; Weisz et al., 2010). The SCSC demonstrated good reliability (Cronbach's $\alpha=.87$ ). The present sample reported scores comparable to a previous study 
of secondary control with a sample of majority young Latinx adolescents $(M=40.32$; Weisz et al., 2010). With no reliability data available for older adolescents, comparison analyses were conducted between younger (11-14) and older (15-17) adolescents and found no significant differences in SCSC score between the two age groups $t(42)=.75, p$ $=.459$.

Independent samples t-tests indicated that gender was related to PSWQ-C such that girls reported significantly more worry than boys, $t(45)=-2.91, p=.006$. Child gender was also related to BAI-Y scores such that girls reported significantly more anxiety than boys, $t(45)=-2.32, p=.025$. Child's gender was not significantly related to any other study variables and was therefore held as a covariate only for analyses including the PSWQ-C or BAI-Y. Child age and grade were not significantly related to any study variables. Parent/guardian acculturation was not significantly correlated with any outcome variable and therefore was not used as a control in hypothesis testing. Due to the two different consenting procedures for the study, independent samples t-tests were conducted to explore any significant differences between the groups. There were no significant differences in demographic variables. There was only one significant difference in outcome variables, such that those who completed the consent/assent electronically $(M=30.67)$ reported significantly higher BAI-Y scores than those who completed the consent/assent in person with the study coordinator $(M=15.29), t(45)=-$ 2.64, $p=.011$. The difference is noted. although the mean of the electronic completion group is impacted by an outlier due to the small sample size, making this difference difficult to interpret meaningfully. 
Model assumptions. Normality of the variables was assessed by inspecting histograms and through use of skewness and kurtosis z-scores. The Americanism subscale exhibited a negative skew and the BAI-Y and IUSC exhibited positive skew. Skew on all three measures was appropriately reduced using logarithmic transformation. Transformed variables were used for hypothesis testing. All other questionnaire scores were normally distributed.

\section{HYPOTHESIS TESTING}

Hypothesis 1: Acculturation predicts fatalism. Bivariate correlations conducted between BIQ and MFS subscale scores are displayed in Table 3.

BIQ-Hispanicism was related to MFS-Luck, $r(47)=-.31, p=.037$. There were no other significant correlations between BIQ and MFS subscales. Linear regressions were conducted to analyze predictive value between all BIQ subscales and the MFS subscale of interest, Fatalism. None of the four BIQ subscales significantly predicted MFS-

Fatalism scores, BIQ-Americanism: $R^{2}=.06, F(1,45)=3.06, \beta=2.92, p=.087$, BIQHispanicism $R^{2}=.00, F(1,45)=.003, \beta=.004, p=.959$, BIQ-Biculturalism $R^{2}=.07$, $F(1,45)=3.27, \beta=.11, p=.077$, and BIQ-Involvement $R^{2}=.05, F(1,45)=2.47, \beta=-$ $.09, p=.123$. 
Table 3

Correlation Coefficients Between BIQ and MFS subscales

\begin{tabular}{|c|c|c|c|c|c|c|c|c|c|}
\hline & \multicolumn{4}{|c|}{ Acculturation } & \multicolumn{4}{|c|}{ Multidimensional Fatalism } & \multirow[b]{2}{*}{ Internality } \\
\hline & Am. & Hisp. & $\mathrm{Bi}$. & Inv. & Fatalism & Helpless & Luck & $\mathrm{DC}$ & \\
\hline \multicolumn{10}{|l|}{ Americanism } \\
\hline Hispanicism & -.11 & & & & & & & & \\
\hline Biculturalism & $.51 * *$ & $.74 * *$ & & & & & & & \\
\hline Involvement & $-.63 * *$ & $.78 * *$ & .15 & & & & & & \\
\hline Fatalism & .25 & .01 & .26 & -.29 & & & & & \\
\hline Helplessness & .05 & -.20 & -.05 & -.24 & $.31 *$ & & & & \\
\hline Luck & -.03 & $-.31 *$ & -.22 & -.24 & .14 & $.73 * *$ & & & \\
\hline Divine Control & .25 & .02 & .26 & -.21 & $.73 * *$ & .26 & .10 & & \\
\hline Internality & .07 & .11 & .14 & .02 & $.51 * *$ & .00 & .03 & .22 & \\
\hline
\end{tabular}

Note. Am. = Americanism subscale of Bicultural Involvement Questionnaire; Hisp. $=$ Hispanicism subscale of Bicultural Involvement Questionnaire; Bi. = Biculturalism subscale of Bicultural Involvement Questionnaire; Inv. = Involvement subscale of Bicultural Involvement Questionnaire; DC = Divine Control subscale of the Multidimensional Fatalism Scale.

$* p<.05, * * p<.01$ 
Hypothesis 2: Fatalism predicts anxiety and worry. Bivariate correlations conducted between MFS, BAI-Y, and PSWQ-C scores, and covariates are displayed in Table 4. MFS-Helplessness was related to BAI-Y score, $r(47)=.52, p<.01$ and PSWQC score, $r(47)=.43, p<.01$. There were no other significant correlations between MFS subscale scores and BAI-Y and PSWQ-C scores. Hierarchical linear regressions were conducted to analyze if MFS-Fatalism scores are predictive of BAI-Y or PSWQ-C scores. Analyses indicated MFS-Fatalism significantly predicted PSWQ-C scores, $R^{2}=$ $.16, F(2,44)=4.08, \beta=.39, p=.024$, but not BAI-Y scores, $R^{2}=.07, F(2,44)=1.74, \beta$ $=-.01, p=.188$. 
Table 4

Correlation Coefficients Between Fatalism, Anxiety Measures, and Covariates

\begin{tabular}{|c|c|c|c|c|c|c|c|c|c|}
\hline & \multicolumn{2}{|c|}{ Covariate } & \multirow{2}{*}{$\begin{array}{l}\text { Anxiety } \\
\text { PSWQ-C }\end{array}$} & \multicolumn{6}{|c|}{ Multidimensional Fatalism } \\
\hline & Gender & BAI-Y & & IUSC & Fatalism & Helpless & Luck & $\mathrm{DC}$ & Internality \\
\hline Gender & & & & & & & & & \\
\hline BAI-Y & .26 & & & & & & & & \\
\hline PSWQ-C & $.36^{*}$ & $.75 * *$ & & & & & & & \\
\hline IUSC & .24 & $.72 * *$ & $.80 * *$ & & & & & & \\
\hline Fatalism & .08 & -.06 & .19 & .22 & & & & & \\
\hline Helplessness & $.30^{*}$ & $.52 * *$ & $.43 * *$ & $.59 * *$ & $.31^{*}$ & & & & \\
\hline Luck & .20 & .28 & .12 & $.46^{* *}$ & .14 & $.73 * *$ & & & \\
\hline Divine Control & .02 & -.13 & .07 & .08 & $.73 * *$ & .26 & .10 & & \\
\hline Internality & .07 & .09 & .22 & $.31 *$ & $.51 * *$ & .00 & .03 & .22 & \\
\hline
\end{tabular}

Note. BAI-Y = Beck Anxiety Inventory- Youth; PSWQ-C = Penn State Worry Questionnaire- Child Version; IUSC = Intolerance of Uncertainty Scale for Children; DC = Divine Control subscale of the Multidimensional Fatalism Scale.

$* p<.05, * * p<.01$ 
Hypothesis 3: Fatalism predicts intolerance of uncertainty. Bivariate correlations conducted between MFS and IUSC scores are displayed in Table 4. IUSC was related to MFS-Helplessness, $r(47)=.59, p<.01$, MFS-Luck, $r(47)=.46, p<.01$, and MFS-Internality, $r(47)=.31, p<.05$. A linear regression between MFS-Fatalism and IUSC scores indicated no significant predicative value, $R^{2}=.05, F(1,45)=2.31, \beta=.01$, $p=.14$.

\section{Hypothesis 4: Fatalism predicts primary and secondary control. Fatalism} predicts anxiety and worry, moderated by secondary control. Bivariate correlations conducted between MFS, PCSC, and SCSC scores are displayed in Table 5. MFSHelplessness was related to PCSC score, $r(47)=-.57, p<.01$, and SCSC score, $r(47)=-$ $.48, p<.01$. MFS-Luck was related to PCSC score, $r(47)=-.50, p<.01$. Linear regressions indicated MFS-Fatalism did not significantly predict PCSC score, $R^{2}=.004$, $F(1,43)=.187, \beta=-.14, p=.667$, or SCSC score, $R^{2}=.000, F(1,42)=.003, \beta=-.018, p$ $=.959$.

Table 5

Correlation Coefficients between Fatalism and Control Measures

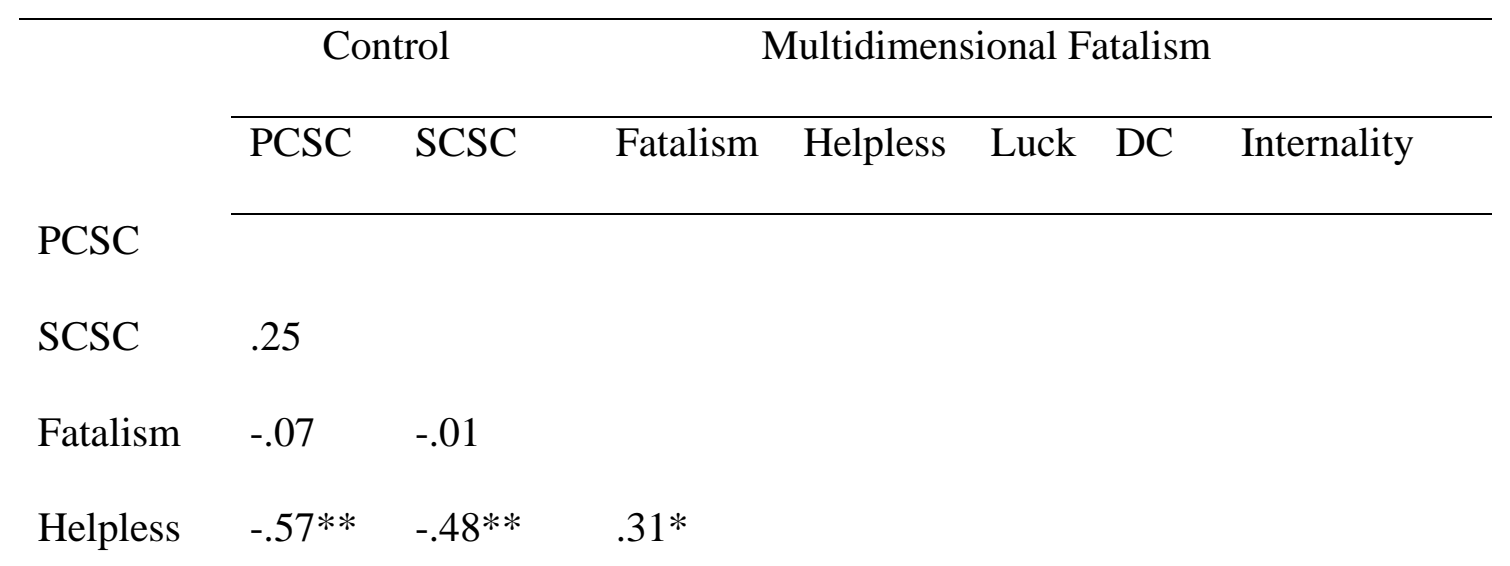




$\begin{array}{lllllll}\text { Luck } & -.50 * * & -.17 & .14 & .73 * * & & \\ \text { DC } & -.08 & .00 & .73 * * & .26 & .10 & \\ \text { Internality } & .23 & .11 & .51 * * & .00 & .03 & .22\end{array}$

Note. PCSC = Perceived Control Scale for Children; SCSC = Secondary Control Scale for Children; DC = Divine Control subscale of the Multidimensional Fatalism Scale.

$* p<.05, * * p<.01$

Bivariate correlations conducted between PCSC, SCSC, BAI-Y, and PSWQ-C scores are displayed in Table 6. SCSC score was related to BAI-Y score, $r(45)=-.61, p<$ .01 , and PSWQ-C score, $r(45)=-.71, p<.01$.

Moderation models were tested predicting anxiety from fatalism with secondary control as a moderator, with gender as a covariate due to previous finding of gender being significantly correlated with BAI-Y and PSWQ-C scores. Secondary control was a significant moderator, $\Delta R^{2}=.1038, F(1,39)=8.07, p=.007$, in the relation between MFS-Fatalism and BAI-Y score. Results are displayed in Figure 1. Secondary control, $\Delta R^{2}=.0263, F(1,39)=2.80, p=.103$, failed to moderate the relation between MFSFatalism and PSWQ-C score.

Table 6

Correlation Coefficients between Control and Anxiety Measures

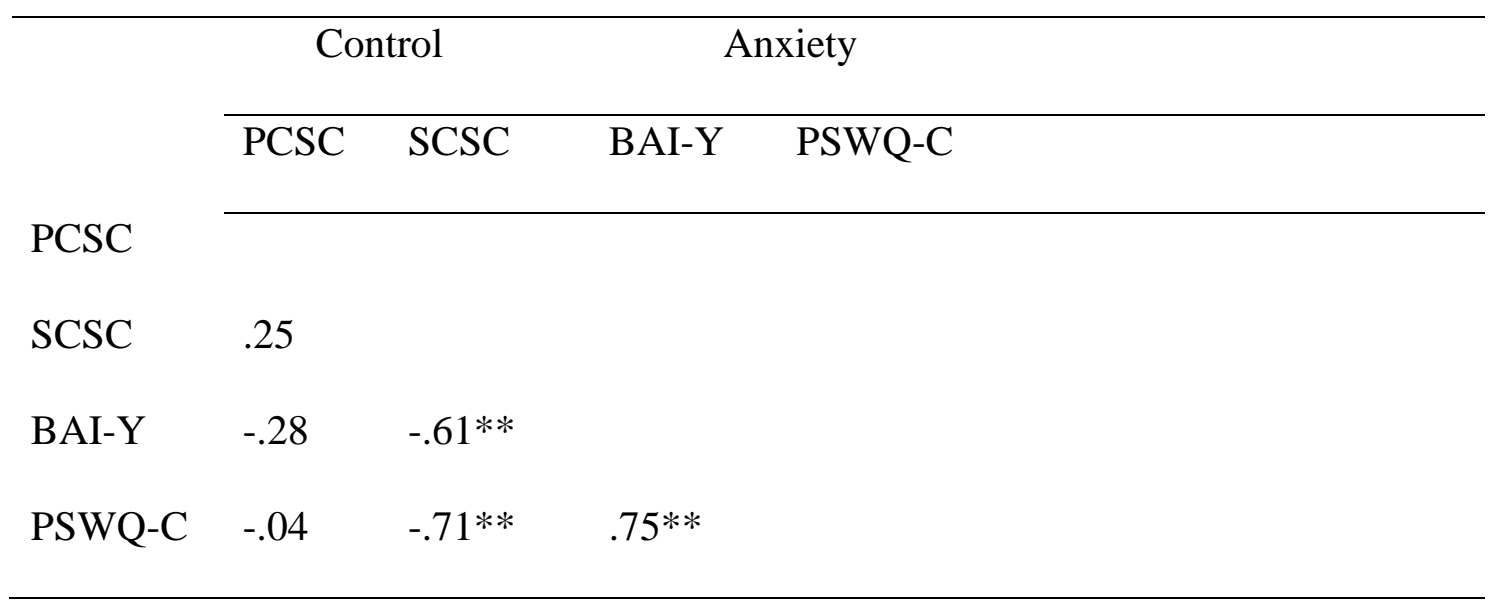


Note. PCSC $=$ Perceived Control Scale for Children; SCSC $=$ Secondary Control Scale for Children; BAI$\mathrm{Y}=$ Beck Anxiety Inventory for Youth; PSWQ-C $=$ Penn State Worry Questionnaire for Children $* p<.05, * * p<.01$

Figure 1. Secondary Control Moderates Relation of Fatalism and Anxiety

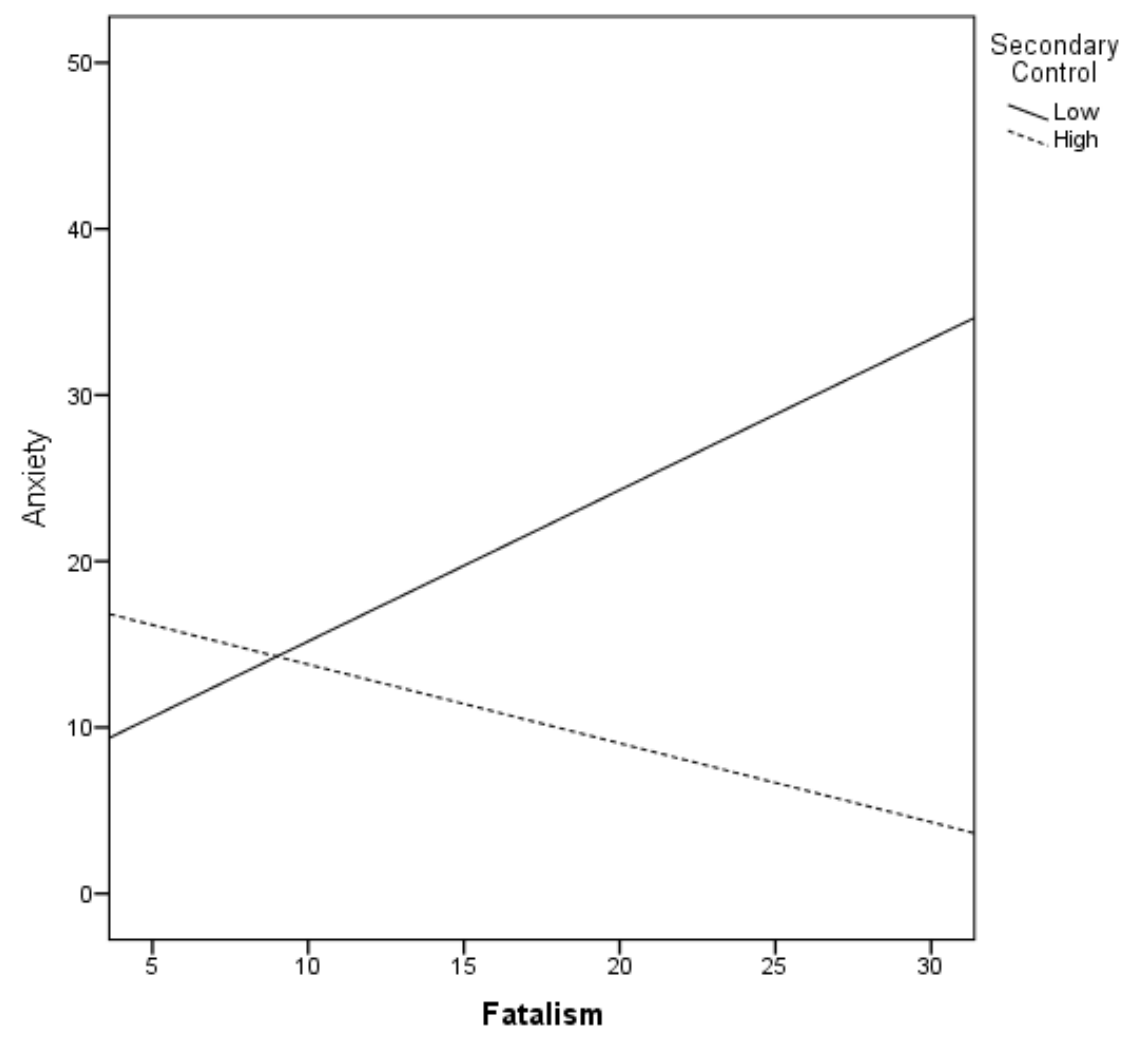

EXPLORATORY ANALYSES

Helplessness. Bivariate correlations indicated the helplessness subscale of the MFS could be an important variable due to significant correlations with anxiety, worry, IU, primary control, and secondary control. Helplessness was also significantly correlated with gender. Independent samples t-tests indicated a significant difference in helplessness by gender such that girls $(M=15.96, S D=5.63)$ reported significantly more helplessness than boys $(M=12.33, S D=4.27), t(44)=-2.33, p=.024$. One individual was excluded from the analysis as they identified as "trans." Results of linear regression analyses for 
MFS-Helplessness predicting outcomes variables are displayed in Table 7. MFS-

Helplessness significantly predicted BAI-Y, IUSC, PCSC, and SCSC scores for girls.

MFS-Helplessness significantly predicted IUSC and PCSC scores for boys.

Table 7

Results of Regression Analyses for Helplessness Predicting Outcome Variables

\begin{tabular}{|c|c|c|c|c|c|}
\hline Variable & $R^{2}$ & b & $F$ & $d f$ & $p$ \\
\hline \multicolumn{6}{|l|}{ BAI-Y } \\
\hline Girls & .32 & .57 & 12.26 & 1,26 & $.002 * *$ \\
\hline Boys & .04 & .20 & 0.66 & 1,16 & .428 \\
\hline \multicolumn{6}{|l|}{ PSWQ-C } \\
\hline Girls & .11 & .34 & 3.35 & 1,26 & .079 \\
\hline Boys & .13 & .35 & 2.30 & 1,16 & .149 \\
\hline \multicolumn{6}{|l|}{ IUSC } \\
\hline Girls & .29 & .54 & 10.58 & 1,26 & $.003 * *$ \\
\hline Boys & .32 & .57 & 7.55 & 1,16 & $.014^{*}$ \\
\hline \multicolumn{6}{|l|}{ PCSC } \\
\hline Girls & .30 & -.55 & 10.70 & 1,26 & $.003 * *$ \\
\hline Boys & .36 & -.60 & 8.34 & 1,16 & $.011^{*}$ \\
\hline \multicolumn{6}{|l|}{ SCSC } \\
\hline Girls & .25 & -.50 & 7.99 & 1,26 & $.009 * *$ \\
\hline Boys & .15 & -.39 & 2.67 & 1,16 & .123 \\
\hline
\end{tabular}

Note. BAI-Y = Beck Anxiety Inventory for Youth; PSWQ-C = Penn State Worry Questionnaire for Children; IUSC = Intolerance of Uncertainty Scale for Children; PCSC $=$ Perceived Control Scale for Children; SCSC $=$ Secondary Control Scale for Children $* p<.05, * * p<.01$

Four Acculturation Groups and Fatalism. To further explore hypothesis 1 of acculturation predicting fatalism, we conducted exploratory analyses using a median split to distinguish low and high levels of both Hispanicism and Americanism. A median split was used in the foundational study that developed the BIQ and therefore used in the present study (Szapocznik et al., 1980). Descriptive statistics are displayed in Table 8. 
Table 8

Fatalism Mean by Acculturation Group

\begin{tabular}{|l|llll|}
\multicolumn{1}{l}{ Hispanicism } & Americanism & M & SD & N \\
\hline \multirow{3}{*}{ Low } & Low & 18.73 & 3.01 & 15 \\
& High & 20.13 & 3.87 & 8 \\
& Total & 19.22 & 3.32 & 23 \\
\hline \multirow{3}{*}{ High } & Low & 22.14 & 6.49 & 7 \\
& High & 17.24 & 4.63 & 17 \\
& Total & 18.67 & 5.58 & 24 \\
\hline \multirow{3}{*}{ Total } & Low & 19.82 & 4.55 & 22 \\
& High & 18.16 & 4.53 & 25 \\
& Total & 18.94 & 4.57 & 47 \\
\hline
\end{tabular}

A two-way ANOVA was conducted that examined the effect of Hispanicism and Americanism level on fatalism. There was a statistically significant interaction between the effects of Hispanicism and Americanism level on fatalism, $F(1,43)=5.241, \mathrm{p}=.027$. Results are displayed in Figure 2. Post hoc linear regressions were performed to analyze predictive value of Americanism to fatalism by level of Hispanicism. Analyses revealed Americanism is a significant negative predictor of fatalism for the high Hispanicism group $\left(R^{2}=.239, F(1,22)=6.904, \beta=-.31, p=.015\right)$ but not for the low Hispanicism $\operatorname{group}\left(R^{2}=.002, F(1,21)=.039, \beta=-.03, p=.844\right)$. 
Figure 2. Interaction of Americanism and Hispanicism Levels on Fatalism

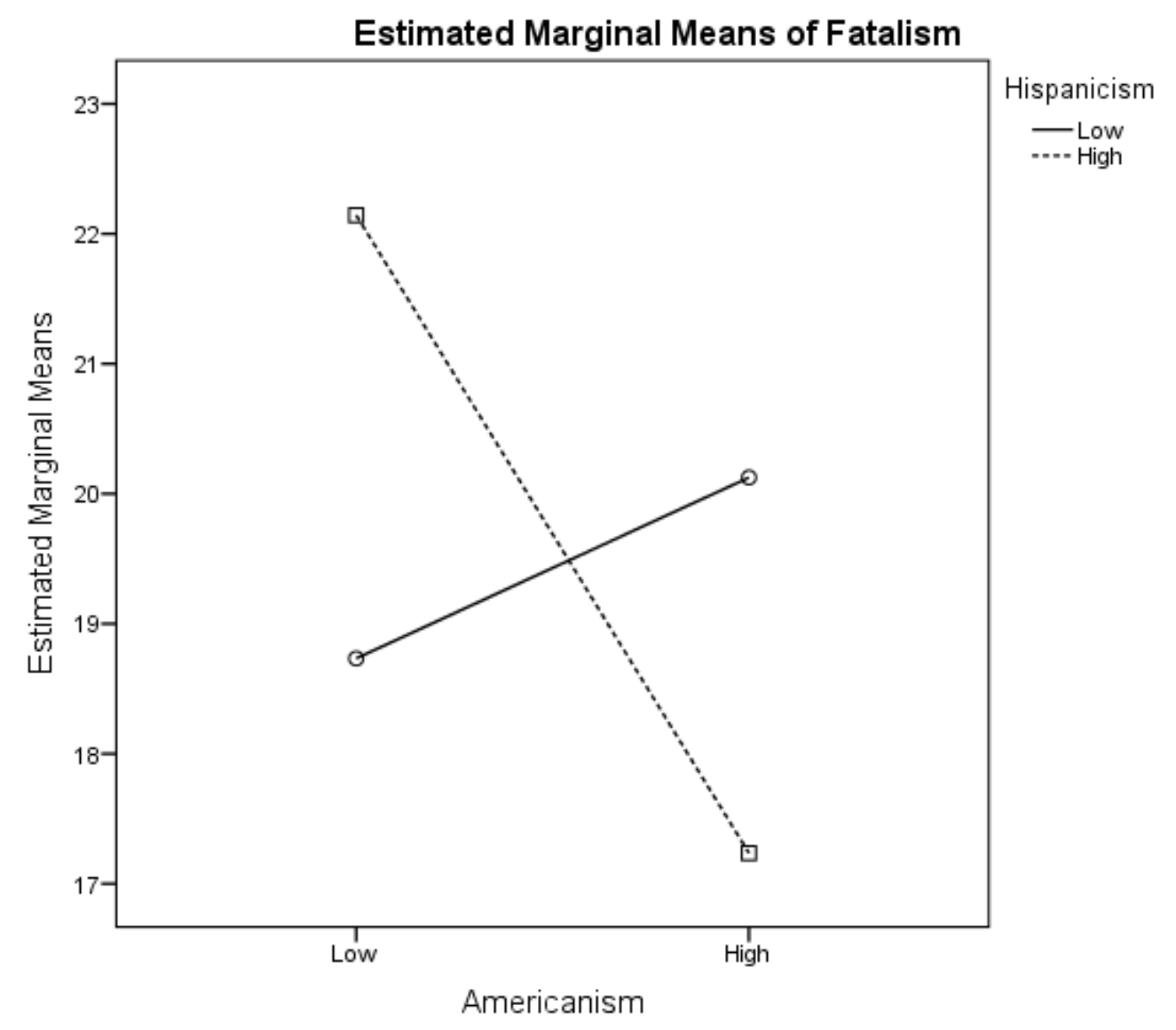

Four Acculturation Groups and Anxiety and Worry. Given the results of exploratory analyses that supported a four-part model of acculturation, similar analyses were used to investigate differences in anxiety and worry. A two-way ANOVA was conducted that examined the effect of Hispanicism and Americanism level on anxiety, measured by BAI-Y scores, and worry, measured by PSWQ-C scores, with gender held as a covariate. There was not statistically significant interaction between the effects of Hispanicism and Americanism level on anxiety, $F(1,42)=0.693, \mathrm{p}=.410$. Descriptive statistics are displayed in table 9. Results of the two-way ANOVA are displayed in Figure 3. There was a significant interaction for worry, $F(1,42)=4.954, \mathrm{p}=.031$. 
Descriptive statistics are displayed in table 10. Results of the two-way ANOVA are displayed in Figure 4.

Table 9

Anxiety Mean by Acculturation Group

\begin{tabular}{|l|llll|}
\multicolumn{1}{l}{ Hispanicism } & Americanism & M & SD & N \\
\hline \multirow{3}{*}{ Low } & Low & 15.27 & 9.07 & 15 \\
& High & 20.25 & 15.82 & 8 \\
& Total & 17.00 & 11.74 & 23 \\
\hline \multirow{3}{*}{ High } & Low & 24.14 & 17.65 & 7 \\
& High & 14.76 & 9.93 & 17 \\
& Total & 17.50 & 13.00 & 24 \\
\hline \multirow{3}{*}{ Total } & Low & 18.09 & 12.72 & 22 \\
& High & 16.52 & 12.07 & 25 \\
& Total & 17.26 & 12.26 & 47 \\
\hline
\end{tabular}


Figure 3. Interaction of Americanism and Hispanicism Levels on Anxiety

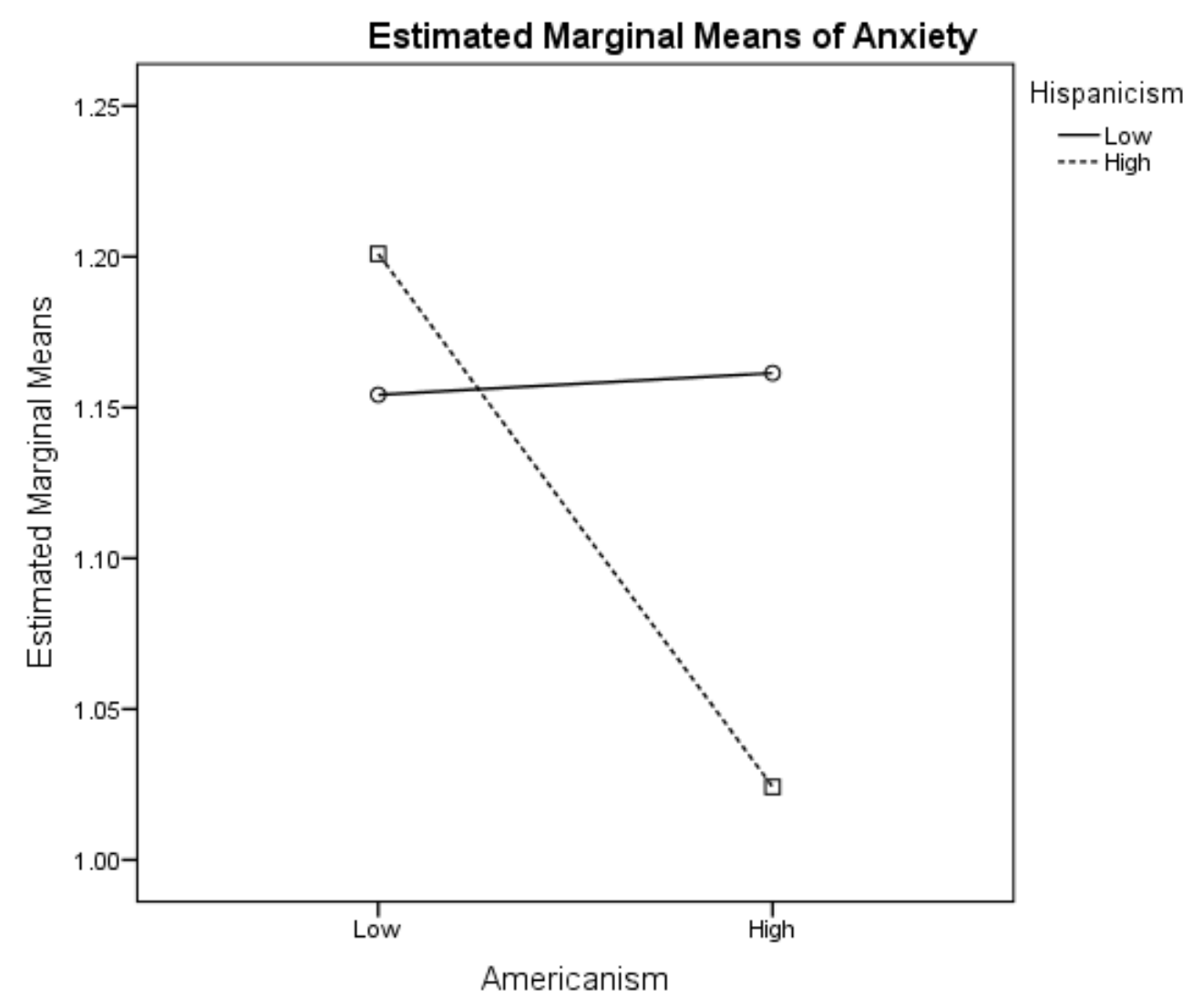

Table 10

Worry Mean by Acculturation Group

\begin{tabular}{|l|llll|}
\multicolumn{1}{l|}{ Hispanicism } & Americanism & M & SD & N \\
\hline \multirow{3}{*}{ Low } & Low & 18.27 & 8.79 & 15 \\
& High & 25.88 & 12.68 & 8 \\
& Total & 20.91 & 10.63 & 23 \\
\hline \multirow{3}{*}{ High } & Low & 22.57 & 10.41 & 7 \\
& High & 14.18 & 10.68 & 17 \\
& Total & 16.63 & 11.08 & 24 \\
\hline \multirow{3}{*}{ Total } & Low & 19.64 & 9.25 & 22 \\
& High & 17.92 & 12.41 & 25 \\
& Total & 18.72 & 10.96 & 47 \\
\hline
\end{tabular}


Figure 4. Interaction of Americanism and Hispanicism Levels on Worry

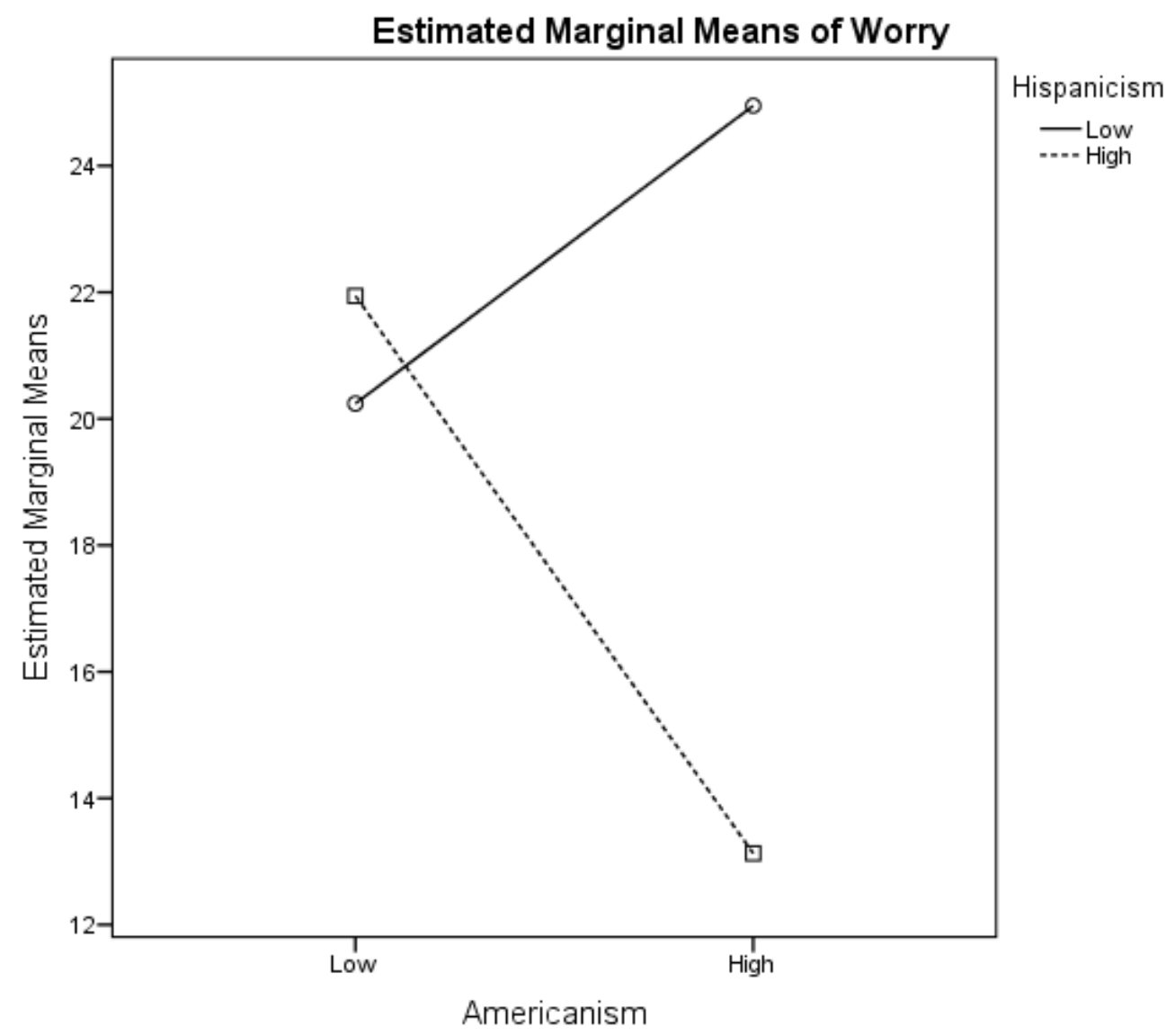

Post hoc linear regressions were performed to analyze predictive value of Americanism to worry by level of Hispanicism. Analyses revealed Americanism is not a significant predictor of worry for the high Hispanicism group $\left(R^{2}=.030, F(1,22)=.689\right.$, $\beta=-.17, p=.416)$ or the low Hispanicism group $\left(R^{2}=.098, F(1,21)=2.27, \beta=.31, p=\right.$ $.147)$.

Fatalism and IU by Age Split. To further explore hypothesis 3 which, in original hypothesis testing found fatalism to not be a significant predictor of IU, a median age split was applied to analyze age groups separately. This was informed by developmental models of executive function, hypothesizing a certain level of developmental maturity is 
necessary in order to engage in higher-level future-oriented thinking such as what would be required to have a concept of IU and fatalism. Linear regression analysis for adolescents ages 12-14 revealed fatalism was not a significant predictor of IU, $R^{2}=.001$, $F(1,26)=0.02, \beta=.025, p=.90$. Linear regression analysis for the older adolescents, ages 15-17, revealed fatalism was a significant predictor of IU, $R^{2}=.23, F(1,19)=5.37$, $\beta=.479, p=.03$. 


\section{DISCUSSION}

The current study examined the role of Latino/a culture on anxiety and associated variables. Specifically, the present study investigated fatalism, a belief that fate determines the future, which previous studies have shown to be prevalent in the Latino/a population. The extant literature has elucidated a lower prevalence of anxiety disorders in the Latino/a population as compared to the non-Latino/a White population as well as an increasing rates of anxiety disorders as acculturation increases. It remains unknown, however, the mechanism within acculturation that changes the experience of anxiety. Fatalism was explored as a potential contributor given its shared themes of control and future-orientation with anxiety and worry.

The aim of the present study was to fill a gap in the research by examining the influence of one Latino/a cultural variable on anxiety and worry. The study hypothesized fatalism is related to the anxiety and worry process through factors of intolerance of uncertainty and control. Specifically, it was hypothesized that adolescents that more closely identified with the Latino/a culture would report higher levels of fatalism and subsequently would exhibit lower intolerance of uncertainty, anxiety, and worry. It was also hypothesized that fatalism would predict lower primary control, but higher secondary control. Finally, it was hypothesized secondary control would moderate the relation between fatalism and anxiety and worry such that as secondary control increases, 
the relationship between fatalism and anxiety and worry also increases in a negative direction.

\section{Preliminary Analyses}

Preliminary analyses revealed the current sample was primarily female, of Mexican descent, and Catholic. Additionally, most of the adolescents were born in the U.S. and have at least one immigrant parent. Self-reported family income was very low, with approximately half either at or below the poverty line, though an exact assessment is not possible as information on the number of persons in the household was not collected.

The current sample showed lower levels of Hispanicism than seen in previous studies. The lower levels of involvement and engagement in Latino/a culture in this sample can partially be explained by recruitment procedures, namely the exclusion of individuals not fluent in the English language. Comparison samples, on the contrary, were made up of a mix of English and Spanish speakers (Martinez et al., 2018).

The current sample demonstrated a low endorsement of anxiety, as measured by the BAI-Y, in terms of the associated severity index; however, this low anxiety is comparable to previous studies using a non-clinical sample. Given that the present study analyzed anxiety on a continuum as opposed to categorizing those with and without an anxiety disorder, paired with the low levels of anxiety endorsed, interpretations about the effects of study variables on anxiety disorders should be limited.

There were significant gender differences in self-reported anxiety and worry. This finding was expected and is in accordance with the extant literature on gender, anxiety, and worry (McLean, Asnaani, Litz, \& Hofmann, 2011; Romero-Acosta, Penelo, Noorian, Ferreira, \& Domènech-Llaberia, 2014). Though the reason behind the gender gap in 
anxiety disorders lacks a comprehensive explanation, there is evidence temperamental factors of negative affectivity and neuroticism are influential (King, Ollendick, \& Gullone, 1991; Steiner, Ryst, Berkowitz, Gschwendt, \& Koopman, 2002). In addition to these trait characteristics, there appears to be a bidirectional relation with gender socialization that further exacerbates the expression of anxiety disorders (McLean et al., 2011).

There was a significant difference in self-reported anxiety between the group who did consent/assent procedures verbally $(\mathrm{N}=41)$ with the study coordinator and those who completed the process electronically without assistance $(\mathrm{N}=6)$, such that the latter reported significantly higher levels of anxiety. Interpretations about these differences are limited by the great difference in sample size between the two groups. Potential explanations include the absence of the study coordinator in the process resulting in increased candidness from the participants and skewed recruitment from physicians and community partners identifying only adolescents known to have higher levels of anxiety. Ultimately, both groups completed every data collection measure in the same manner and therefore this difference was not controlled for in proceeding analyses.

\section{Hypothesis Testing}

Acculturation and fatalism. Acculturation was measured using a bicultural measure, as opposed to a binary measure, to facilitate assessment of Latino/a and American cultural practices independently and concurrently. A bicultural model better represents the interplay of two cultures the individual is contending with than does a single continuum in which one can only be highly involved in one culture when low in the other (Romero et al., 2020). The current study hypothesized higher levels of Latino/a 
cultural involvement, or Hispanicism as was termed by the measure used, would be related to higher endorsement of fatalism. Conversely, we hypothesized higher levels of American cultural involvement, or Americanism, would be related to lower endorsement of fatalism. These paired hypotheses were not supported by the regression analyses; there was no significant predictive value of cultural involvement on endorsement of fatalism.

One reason for the unexpected finding could be that by excluding participants that were not fluent in English, we restricted our sample to those on the higher end of Americanism. Similarly, by limiting the sample to those proficient in English, that means anyone who is endorsing the Spanish language items could only be bilingual and not primarily Spanish-speaking.

Exploratory analyses were conducted by splitting acculturation into four groups consisting of pairings of high and low Hispanicism and Americanism. This four-part model of acculturation, as displayed in Figure 2, is consistent with extant literature labeling the four strategies as marginalization, separation, assimilation, and integration (Berry, 2005). These four strategies of acculturation are defined by the individual's preference of or involvement in their heritage culture and host culture. Marginalization occurs when the individual is not engaged in or enjoying aspects of either culture. They are deemed marginalized because they are not an active member in either community, the heritage or the host. These individuals are likely to be experiencing a high level of cultural conflict that they resolve by having minimal involvement in both cultures (Berry, 2005). For the purposes of the cultures being investigated in this study, a marginalized individual would be low in both Hispanicism and Americanism. The separation strategy of acculturation is characterized by a strong preference for the heritage culture over the 
host culture. These are individuals who retain their culture of origin and are not active in the cultural practices of the location where they are now located, therefore being deemed separated. Separation in this study is demonstrated by high Hispanicism and low Americanism. Assimilation describes an individual who prefers, or is more active in, the host culture over their heritage culture. The individual is assimilated to the culture they are currently in and forego or reject their culture of origin. Assimilated individuals in this study are low in Hispanicism and high in Americanism. Finally, integration occurs when the individual is involved in, and enjoys, both their heritage culture and the host culture. These individuals integrate the two cultures they are exposed to. These individuals can also be thought of as being high in biculturalism. Study participants high in both Hispanicism and Americanism fall into the integrated category.

A two-way ANOVA comparing differences in fatalism by the four acculturation groups resulted in significant findings. In splitting the groups, we found that most participants fell in the integration group $(\mathrm{N}=17)$, followed by marginalization $(\mathrm{N}=15)$, assimilation $(\mathrm{N}=8)$, and separation $(\mathrm{N}=7)$. Integration being the most common identification is consistent with previous studies but marginalization being a close second is inconsistent (Berry, Phinney, Sam, \& Vedder, 2006). In relation to fatalism, the order of acculturation group from most to least fatalism is separation, assimilation, marginalization, and integration. The significant interaction was such that Americanism predicted lower fatalism only in the high Hispanicism group. Americanism was not a significant predictor of fatalism in the low Hispanicism group. Meaning, adolescents who did not reduce in Hispanicism through acculturation but did additively gain in Americanism reported the lowest levels of fatalism. This can be interpreted as a 
representation of culture learning in which the adolescent learns American cultural values that are contrary to fatalism, such as individualism (Hirschman, 2003).

Berry (2005) emphasized the importance of two factors when discussing acculturation: preference and behavior change. Individuals may be congruent in their culture preferences and practices, but often they are not. This is important because the measure used in the present study, the Bicultural Involvement Questionnaire, may be a misnomer in that it does not measure behavior and only inquires on preference. The measure comes close to asking about behavior with items on how comfortable the individual feels speaking each language; however, by using the word comfortable, the measure is then assessing preference or affect, not behavior per se. Alternatively, to capture behavior, the measure would need to ask the frequency in which the individual engages in cultural practices, which it does not. By using the Bicultural Involvement Questionnaire, the present study can only speak to one of the two components of acculturation.

The BIQ's emphasis on preference for cultural practices also excludes two other important aspects of acculturation: values and identity. Cultural practices, values, and identifications have been posited as the three domains of acculturation that match up to the behavioral, cognitive, and affective changes an individual undergoes (Schwartz, Unger, Zamboanga, \& Szapocznik, 2010). Although these three domains of acculturation are connected, they are independent processes and have been found to be only modestly intercorrelated (Schwartz et al., 2011). This is important because, although it has not been definitively defined as such, fatalism most closely fits in the values/cognitive category. By using a measure of cultural practices in the present study, we did not assess the 
cognitive changes of acculturation most relevant to fatalism; however, this brings its own difficulties as cultural values measurement is often done with an individualism versus collectivism perspective that may not best capture the concept of fatalism. Having measured cultural practices and not values is also important because there is evidence that cultural practices are the first of the three domains to change in acculturation (Schwartz et al., 2015). Therefore, it may be that an adolescent who endorsed high Americanism in cultural practice, may not be as high in Americanism in cultural values, confounding the analyses in relation to fatalism.

Figure 5. Four Strategies of Acculturation

Hispanicism

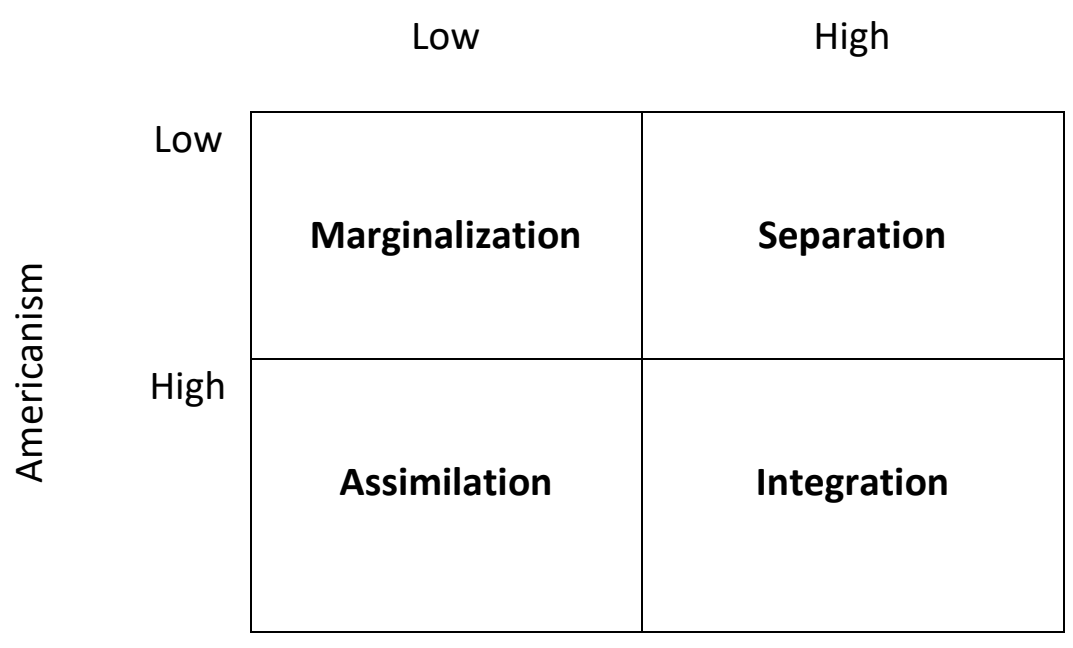

Fatalism and anxiety and worry. The current study hypothesized fatalism would be significantly related to anxiety and worry. Specifically, it was hypothesized fatalism would be negatively related to anxiety and worry. These hypotheses were not supported, and in fact, there was a significant finding in the opposite direction in which fatalism positively predicted worry. These findings demonstrate an interesting split in the physical and cognitive symptoms of anxiety. It may be that because fatalism is also 
largely a cognitive process, the relation to the cognitive presentation of anxiety (worry) is most obvious. The cognitive connection, however, does not appear to be related to the broader experience of anxiety that includes the physical symptoms. It could be that fatalism and worry are similar cognitive processes, but fatalism is not resulting in the heightened physiological arousal characteristic of anxiety disorders. In other words, fatalism may produce more cognitive content related to worry but is not distressing. Exploratory analyses were conducted by splitting acculturation into four groups consisting of pairings of high and low Hispanicism and Americanism as aforementioned. Results indicated a significant interaction but not significant findings in Americanism predicting worry for either Hispanicism group. Meaning, there were significant differences in the direction of the relation between Americanism and worry by Hispanicism group, but when analyzed independently, Americanism did not have a significant predictive value.

In relation to worry, the order of acculturation group from most to least most is assimilation, separation, marginalization, and integration. The integrated group demonstrated the lowest level of worry, which is consistent with previous studies finding the integration strategy of acculturation to be associated with the best psychological outcomes of the four acculturation groups (Berry et al., 2006). The reason for the protective nature of an integration approach to acculturation is posited to be due to the increased ability to choose the behaviors best suited for any given cultural context the individual is in (LaFromboise et al., 1993). For example, an integrated Latino/a adolescent in a predominantly Latino cultural context, such as a quinceañera, is better able to engage in the cultural rituals of the event than is an assimilated Latino/a 
adolescent who may instead reject the rituals and isolate themselves during the event. Assimilation poses the highest risk for heightened worry which demonstrates more adoption of American values alone does not best serve the individual.

Fatalism and IU. Intolerance of uncertainty was measured using the IUSC (Comer et al., 2009), an adaptation of a the Intolerance of Uncertainty Scale (IUS; Buhr \& Dugas 2002) for use with children and adolescents ages 7-17. There is a robust literature detailing the positive relation between IU and anxiety and worry. IU is of particular interest in the present study due to its shared theme of future orientation with fatalism. It was hypothesized fatalism would negatively predict IU such that higher fatalism would result in lower levels of IU. This hypothesis was formulated based on fatalism seemingly challenging the assumptions that compose IU. Specifically, fatalism challenges the IU premise that uncertainty is unfair, stressful, and negative and instead contains the perspective that uncertainty is commonplace and to be expected. The hypothesis was not supported. Initial findings using linear regression indicated no significant predictive value of fatalism on IU.

Non-significant findings spurred further consideration of the impact of developmental level on acquisition of the necessary future orientation for IU and fatalism. Having a framework for making meaning of future events necessitates a certain level of abstraction that may not yet be present without having undergone the formal operational stage of development (Nurmi, 1991). Adolescence marks an important time of transition in cognitive abilities and therefore likely a time of transition in both fatalism and IU. Fatalism and IU require an ability for external focus and planning that is not typically characteristic of younger adolescents, who are more likely to be focused on the 
self and are not yet preoccupied with the possible sources and consequences of future events (Giollabhui et al., 2018). In fact, very early studies suggest adolescent egocentrism does not diminish until age 15 or 16 (Elkind, 1967). Orientation toward the future increases with age (Nurmi, 1991), but varies in timing by individual, meaning some adolescents develop an abstract concept of the future earlier than others (Giollabhui et al., 2018), as would be expected with the varying trajectories of development more broadly.

The age range of the current sample, 12-17, is broad considering the many cognitive developmental processes underway. Recognizing there is not a definitive answer as to when an adolescent develops future orientation, exploratory analyses utilized a median age split to investigate the role of fatalism in IU separately for younger and older adolescents. Results of linear regressions indicated fatalism was not a significant predictor of IU for younger adolescents but was a significant positive predictor of IU for older adolescents. This finding is in the opposite direction of what was hypothesized, meaning higher fatalism predicted higher IU. One possible explanation is that fatalism represents a strategy for making meaning of uncertainty and is more present when an adolescent has a low tolerance for uncertainty and therefore more actively searches for an explanation than those with a high tolerance for uncertainty. The different findings between age groups is important because it can inform when, developmentally, fatalism and IU begin to be meaningful.

Fatalism and Control. Control was operationalized in two distinct forms, primary control and secondary control. It was important to investigate these two forms of control separately as it was hypothesized they would relate differently to fatalism. Fatalism, by definition, assigns control of future events to fate and therefore was 
hypothesized to be negatively related to primary control which is one's perception they control external events. Conversely, fatalism was hypothesized to be positively related to secondary control which is one's perception of control over their psychological responses to outcomes. Results revealed fatalism did not significantly predict primary or secondary control.

These findings indicate fatalism is not best described as a perception of one's control and is instead a more complex concept. If fatalism is conceptualized as a value, it may be that individuals are not in disbelief that they personally have some control of outcomes, but they value having a viewpoint that control is shared with a greater power. In other words, 'fate is in control' and 'I am in control' are not mutually exclusive and can coexist. In fact, it may reflect a healthy recognition of what is within and outside of one's control given the future is a mix of internal and external factors.

A non-significant relation between fatalism and primary control could also reflect a difference in the way each construct was measured. The PCSC inquires on the individual's perception of control over specific outcomes such as grades, relationships with peers, and avoiding punishment. The MFS-F on the other hand, uses broader language, asking about "bad things" happening, life's general unpredictability, and death. Given what has already been discussed about adolescents' future orientation and focus on the immediate self, it coheres why these two concepts do not fall under one cognitive framework. Similarly, fatalism and secondary control operate independently as do primary and secondary control. Meaning, what one thinks will occur in the future and how one believes they will handle future events are separate. 
As an extension of the primary hypotheses for fatalism and variables of control, it was further hypothesized secondary control would moderate the relation between fatalism and anxiety and worry. Specifically, it was hypothesized that higher fatalism will predict lower levels of anxiety and worry when a high level of secondary control is present. Results supported the hypothesis for anxiety but not for worry. Fatalism was related to higher levels of anxiety when secondary control was low and lower levels of anxiety when secondary control was high. Interpreted in the context of the broader discussion of fatalism being a risk or protective factor, it appears the answer is that it depends.

When an individual does not have a positive view of their ability to adapt and respond effectively, an uncertain or uncontrollable future poses a threat. An assumed threat may be, 'if something bad happens, I will not be able to emotionally withstand it and will fall into a deep despair'. Or, 'if something bad happens, I will be unable to adapt and will be disconnected from my social group.' Decades of research has demonstrated the effect of threat perception on anxious arousal through activation of the sympathetic nervous system (e.g., Dalton, Kalin, Grist, \& Davidson, 2005; McCaul, Solomon, \& Holmes, 1979). Therefore, when fatalism is perceived as a threat, it leads to the increased physiological arousal captured by the BAI-Y. Alternatively, when secondary control is present, fatalism is not perceived as a threat and instead may be the healthy acceptance it was hypothesized to be in the present study.

Secondary control did not moderate the relation between worry and fatalism. As was previously discussed, fatalism is independently predictive of worry and with this extended analysis it was revealed secondary control does not significantly influence that 
relation. One possible explanation is that fatalism's effect on worry is not perceived as something necessary to actively cope with and is instead a normative experience.

Helplessness. Exploratory analyses were used to further investigate helplessness, a subscale of the MFS measure, due to the many significant correlations discovered in preliminary analyses. There was a significant gender difference in helplessness such that girls reported significantly more helplessness than boys. This relation may be indicative of the Latino/a cultural standard for women and girls named Marianismo which, among other aspects, includes a practice of feminine passivity and sacrificing one's own needs for that of her family (Gil \& Vasquez, 1996). Marianismo is also related to self-silencing and subordination to others and without a voice or power to act, is easily conceptualized as helplessness (Piña-Watson, Castillo, Jung, Ojeda, \& Castillo-Reyes, 2014). Given the previous findings suggesting culture may be influencing Latina girls' experience of anxiety and the interest of cultural influence in the present study, the following analyses were conducted separately for girls and boys. This allows for conclusions about unique gender presentations of anxiety for Latino/a adolescents as would not be possible if simply controlling for gender in the analysis.

Results revealed helplessness was a significant predictor of IU and primary control for both boys and girls and of anxiety and secondary control only for girls. For girls, higher endorsement of helplessness was related to more anxiety and less secondary control. This is a novel finding as previous studies have concluded helplessness is related to anxiety and coping, but have not reported different effects by gender (Waschbusch, Sellers, LeBlanc, \& Kelley, 2003). This may mean the experience of helplessness is somehow more conceptually relevant to anxiety and coping for girls, potentially with 
direct relations to constructs of gender socialization, such as inhibition and assertiveness (McLean \& Anderson, 2009). It may also be that the experience of helplessness is more impactful for girls, potentially compounding upon their minority status as female which already comes with a level of powerlessness.

\section{IMPLICATIONS}

Investigating concepts related to future orientation, such as fatalism, is particularly important in relation to adolescence given the critical period of making decisions about one's life. In adolescence, many individuals are beginning to choose their careers and future families, both of which have a great impact on later adult life (Nurmi, 1991). As it relates to culture, adolescence is also a period when many are developing their identity as an individual and deciding which culture(s) to further explore or commit to with their newfound autonomy.

The more positive outcomes revealed by an integration process of acculturation, represented by lowest rates of anxiety and worry, demonstrate it is important for anyone working with Latino/a adolescents (e.g., clinicians, teachers) to pursue this strategy and not neglect or reject either of the two cultures in which the adolescent exists. This includes refraining from pathologizing or seeking to eliminate cultural values, such as fatalism, based on research not conducted on this population or that does not consider cultural norms. More broadly, societally it is imperative we stop propagating messages, via discriminatory policies and interpersonal interactions, that immigrants must wholly adopt traditional American culture and leave behind their heritage culture, including traditions, beliefs, and language. Embracing cultural differences not only fosters integration of cultures but historically tends to lead to increased support at an institutional 
level for the immigrant community, such as ensuring culturally sensitive health care and schooling (Berry, 2005).

\section{LIMITATIONS}

The contributions made to the literature by the present study are to be interpreted within the confines of the study's limitations. First, the cross-sectional method of data collection used precludes conclusions about causality. For example, though it may conceptually make most sense, we are unable to conclude that higher fatalism is resulting in greater worry and not the reverse. The small size of the sample is a limitation of the study. Though there were a number of significant findings, there is still a question of insufficient power to find relations with small effect sizes. This is further complicated by a lack of reference material for what the expected effect size of these analyses should be. As previously reviewed, the literature on fatalism is not extensive and, to our knowledge, this is the first study exploring its connection to anxiety and worry.

There are several factors that contributed to the small sample size. The present study sought to recruit only adolescents within a 6-year age range and from a specific ethnic group that is a small minority of the population in the city where the data were collected. Additionally, due to university regulations for grant funding, participants were mandated to provide a valid social security number to receive payment for participation. This requirement resulted in distrust about the study from potential participants as well as community partners that were asked to assist in recruitment. In fact, an influential community partner decided they would not advertise the study due to this requirement. Partnerships with community organizations that the locals trust is very important in recruiting Latino/as for research studies (Haack, Gerdes, \& Lawton, 2014) and this 
partner's withdrawal from recruitment hindered further data collection. In the future, funded studies using a sample of Latino/a participants should consider options that will not require social security numbers to be collected.

The Latino/a population is diverse and studying the group as a combined unit is complex. The present study consisted of primarily Mexican-heritage adolescents. This is consistent with the statistics of the Latino/a population in the United States. The Cuban population was overrepresented in the current sample, as was to be expected due to the demographics of the city where the data were collected. Unfortunately, but interestingly, because the question about heritage/nationality was open-ended, a significant percentage $(\mathrm{n}=7 ; 14.9 \%)$ reported "American," "USA," or a similar variant. This made it impossible to determine the background of their Latino/a ethnic identity. It is an interesting response, however, because it may be indicative of how distant the individual is from their Latino/a identity, either by distant immigration history (i.e., $2^{\text {nd }}, 3^{\text {rd }}$ generation in the United States) or by acceptance of Latino/a heritage. In fact, of the 7 who responded this way, 6 fell into the marginalized category of acculturation and 1 was in the integrated category. That means more than one-third of the marginalized group self-reported their heritage/nationality as "American" or "USA" despite low involvement in both the Latino/a and American culture. This response was also observed in a previous study in which $4.5 \%$ of Latino/a adolescents in the study sample endorsed "United States" as their ethnic group (Schwartz et al., 2014).

Another limitation of the study is the questionnaire used to measure fatalism, while it demonstrated good reliability, is new and has only been used in the original study of its development. Being that there is no gold-standard measure of fatalism, or consistent 
measurement in previous studies, the present study is somewhat limited in its ability to compare results with the few other studies on fatalism.

\section{SUMMARY AND FUTURE DIRECTIONS}

The current study contributes significantly to the understanding of fatalism's influence on Latino/a mental health in the context of acculturation. Importantly, findings contribute to better defining fatalism, specifically by concluding it is not simply a function of control or equivalent to helplessness, as it had previously been conceptualized.

It is the first study to investigate fatalism in relation to anxiety and associated variables. The results of the present study revealed significant connections between fatalism and anxiety correlates that provide a foundation for future research in this area. Previous studies on fatalism in the Latino/a population failed to properly incorporate cultural context and in doing so weakened their conclusions of fatalism as a risk factor for poor health. The present study revealed the acculturation process in relation to fatalism and anxiety is important and best studied using a four-part model otherwise risking missing significant interactions by failing to account for the complexity of acculturation. As demonstrated, fatalism does not definitively fall into the category of a risk or protective factor and its influence is dependent on other qualities of the individual, specifically their self-confidence in coping. Overall, the current study warrants future research on fatalism, culture, and anxiety.

An interesting question for further conceptual refinement is whether fatalism is best described as a state or trait phenomenon. In other words, the present study did not distinguish whether fatalism can be present in some areas of an individual's life but not 
others; or, if alternatively, it is a stable lens through which the individual views their experiences across domains. Another concept to be considered in future studies seeking to better define fatalism is attribution. Though fatalism was not best defined in relation to primary control in the present study, a more comprehensive framework that accounts for the interplay of internal, external, stable, and unstable causal attributions could elucidate unique relations and/or mediating and moderating variables.

A future study using longitudinal data collection on acculturation, fatalism, and anxiety could better elucidate the findings in the present study and begin to address the state-trait question about fatalism. A study design that collects data at multiple timepoints could allow conclusions about the direction of the relations between these variables. An example of such a study would be one that recruits a large sample of Latino/a adolescents, with quotas for varying levels of acculturation proxies (e.g., U.S. born, foreign-born, language proficiencies) to allow for comparative analyses, and follows them for multiple years, collecting data each year. A data source consisting of multiple time periods would allow for a model of causation and therefore identify if and when intervention would be most appropriate. For example, it could be that earlier acculturation experiences relate to later expression of anxiety disorders and if identified early, can facilitate prevention efforts. A longitudinal study could shine light on the mechanism at play as fatalism changes with acculturation, whether it is shedding of Latino/a culture or learning of American culture.

The present study used self-report questionnaires to measure anxiety and worry on a continuum. A future study that uses a more comprehensive assessment could better infer if findings relate to anxiety disorders. A diagnostic assessment could be used to add 
more complexity by differentiating between various anxiety disorders and the relation of each to acculturation and fatalism. It may be, for example, that fatalism is related to worry-based anxiety disorders but not primarily fear-based anxiety disorders. Similarly, future studies should consider assessing for acculturative stress in addition to level of acculturation, given evidence that the latter may be most robustly related to psychopathology. Acculturative stress may also mediate or moderate significant findings between acculturation and negative psychological outcomes.

Finally, the resilience hypothesis remains a compelling explanation for the differences observed in the Hispanic health paradox. Similar studies to the present one that include other Latino/a cultural characteristics can lead to a multifaceted explication of changes in the acculturation process that promote resilience. Ruiz et al. (2016) proposed a conceptual model in which social networks mediate cultural processes and health advantages. The model uses social networks as an umbrella term under which familismo, simpatia, and respeto lie given they are all interpersonal in nature. Operationalizing these cultural values under one well-researched term may facilitate connections to established theory; however, it should be regarded as a beginning step that is proceeded by separate investigations of each as they are similar but not equivalent. 


\section{REFERENCES}

Abraído-Lanza, A. E., Viladrich, A., Flórez, K. R., Céspedes, A., Aguirre, A. N., \& De La Cruz, A. A. (2007). Commentary: Fatalismo reconsidered: A cautionary note for health-related research and practice with Latino populations. Ethnicity \& Disease, 17, 153-158.

Ai, A. L., Pappas, C., \& Simonsen, E. (2015). Risk and protective factors for three major mental health problems among Latino American men nationwide. American Journal of Men's Health, 9, 64-75. doi:10.1177/1557988314528533

Ai, A. L., Weiss, S. I., \& Fincham, F. D. (2014). Family factors contribute to general anxiety disorder and suicidal ideation among Latina Americans. Womens Health Issues, 24, 345-352.

Alegría, M., Canino, G., Shrout, P. E., Woo, M., Duan, N., Vila, D., .. Meng, X. L. (2008). Prevalence of mental illness in immigrant and non-immigrant U.S. Latino groups. American Journal of Psychiatry, 165, 359-369. doi:10.1176/appi.ajp.2007.07040704

Alegría, M., Takeuchi, D., Canino, G., Duan, N., Shrout, P., Meng, X. L., Vega, W., ... Gong, F. (2004). Considering context, place and culture: the National Latino and Asian American Study. International journal of methods in psychiatric research, 13(4), 208-20.

American Psychiatric Association. (2013). Diagnostic and statistical manual of mental disorders (5th ed.). Arlington, VA: American Psychiatric Publishing. 
Anastasia, E. A., \& Bridges, A. J. (2015). Understanding service utilization disparities and depression in Latinos: The role of fatalismo. Journal of Immigrant and Minority Health, 17, 1758-1764.

Asnaani, A., Richey, J. A., Dimaite, R., Hinton, D. E., \& Hofmann, S. G. (2010). A cross-ethnic comparison of lifetime prevalence rates of anxiety disorders. Journal of Nervous and Mental Disease, 198, 551-555.

Baer, R. D., Weller, S. C., De Alba Garcia, J. G., Glazer, M., Trotter, R., Pachter, L., \& Klein, R. E. (2003). A cross-cultural approach to the study of the folk illness nervios. Culture, Medicine and Psychiatry, 27, 315-337. doi:10.1023/A:1025351231862

Barlow, D. H. (2002). Anxiety and its disorders (2nd ed.). New York, NY: Guilford Press.

Bayles, B. P., \& Katerndahl, D. A. (2009). Culture-bound syndromes in Hispanic primary care patients. International Journal of Psychiatry in Medicine, 39, 15-31. doi:10.2190/PM.39.1.b

Beck, J. S., Beck, A. T., Jolly, J. B., \& Steer, R. A. (2005). Beck youth inventories: for children and adolescents. Psychological Corporation.

Beck, A. T., Epstein, N., Brown, G., \& Steer, R. A. (1988). An inventory for measuring clinical anxiety: Psychometric properties. Journal of Consulting and Clinical Psychology, 56(6), 893-897.

Benavides, R., Bonazzo, C., \& Torres, R. (2006). Parent-child communication: A model for Hispanics on HIV prevention. Journal of Community Health Nursing, 23, 8194. doi:10.1207/s15327655jchn2302_2 
Berna, C., Lang, T. J., Goodwin, G. M., \& Holmes, E. A. (2011). Developing a measure of interpretation bias for depressed mood: An ambiguous scenarios test. Personality and Individual Differences, 51, 349-354. doi:10.1016/j.paid.2011.04.005

Bernard, M., Strasser, F., Gamondi, C., Braunschweig, G., Forster, M., Kaspers-Elekes, K., \& ... Borasio, G. D. (2017). Relationship between spirituality, meaning in life, psychological distress, wish for hastened death, and their influence on quality of life in palliative care patients. Journal of Pain and Symptom Management, 54, 514-522. doi:10.1016/j.jpainsymman.2017.07.019

Berry, J. W. (2005). Acculturation: Living successfully in two cultures. International Journal of Intercultural Relations, 29, 697-712.

Berry, J. W. (2007). Acculturation strategies and adaptation. In J. E. Lansford, K. DeaterDeckard, \& M. H. Bornstein (Eds.), Immigrant families in contemporary society. (pp. 69-82). Guilford Press.

Berry, J. W., Phinney, J. S., Sam, D. L., \& Vedder, P. (2006). Immigrant Youth: Acculturation, Identity, and Adaptation. Applied Psychology: An International Review, 55, 303-332. doi: 10.1111/j.1464-0597.2006.00256.x

Borkovec, T. D. (1994). The nature, functions, and origins of worry. In G. C. L. Davey \& F. Tallis (Eds.), Wiley series in clinical psychology. Worrying: Perspectives on theory, assessment and treatment (pp. 5-33). Oxford, England: John Wiley \& Sons.

Borkovec, T. D., Alcaine, O., \& Behar, E. (2004). Avoidance Theory of Worry and Generalized Anxiety Disorder. In R. G. Heimberg, C. L. Turk, \& D. S. Mennin 
(Eds.), Generalized Anxiety Disorder: Advances in Research and Practice (pp. 77-108). New York: Guilford Press.

Bostean, G. (2013). Does selective migration explain the Hispanic paradox? A comparative analysis of Mexicans in the U.S. and Mexico. Journal of Immigrant and Minority Health, 15, 624-635. doi:10.1007/s10903-012-9646-y

Bridges, A. J. (2008). Service utilization interview. In M.A. de Arellano (Ed.), Trauma, psychological sequelae, and service utilization rates in Hispanic adults. Charleston, SC: Medical University of South Carolina's Health Disparities Research Pilot Program.

Brown, T. A., Di Nardo, P. A., \& Barlow, D. H. (1994). Anxiety Disorders Interview Schedule for DSM-IV. Albany, NY: Graywind.

Cadet, T. J., Berrett-Abebe, J., \& Stewart, K. (2017). Mental health and breast cancer screening utilization among older Hispanic women. Journal of Women and Aging, 29, 163-172. doi:10.1080/08952841.2015.1113726

Carvajal, S. C., Hanson, C. E., Romero, A. J., \& Coyle, K. K. (2002). Behavioral risk factors and protective factors in adolescents: A comparison of Latinos and nonLatino whites. Ethnicity and Health, 7, 181-193. doi:10.1080/1355785022000042015

Centers for Disease Control and Prevention. (2015). Deaths: Final data for 2014, Table 7. Retrieved from http://www.cdc.gov/nchs/fastats/life-expectancy.htm Chambless, D. L., Caputo, G. C., Bright, P., \& Gallagher, R. (1984). Assessment of fear of fear in agoraphobics: The body sensations questionnaire and the agoraphobic 
cognitions questionnaire. Journal of Consulting and Clinical Psychology, 52, 1090-1097.

Chartier, K. G., Carmody, T., Akhtar, M., Stebbins, M. B., Walters, S. T., \& Warden, D. (2015). Hispanic subgroups, acculturation, and substance abuse treatment outcomes. Journal of Substance Abuse Treatment, 59, 74-82. doi:10.1016/j.jsat.2015.07.008

Cho, Y., Frisbie, W. P., Hummer, R. A., \& Rogers, R. G. (2004). Nativity, duration of residence, and the health of Hispanic adults in the United States. International Migration Review, 38, 184-211.

Chorpita, B. F., Albano, A. M., \& Barlow, D. H. (1996). Cognitive processing in children: Relation to anxiety and family influences. Journal of Clinical Child Psychology, 25, 170-176.

Comer, J. S., Roy, A. K., Ph, D., Furr, J. M., Gotimer, K., Beidas, R. S., ... Ph, D. (2009). The Intolerance of Uncertainty Scale for Children: A Psychometric Evaluation, 21, 402-411. doi:10.1037/a0016719.

Costello, E. J., Egger, H. L., \& Angold, A. (2005). The developmental epidemiology of anxiety disorders: Phenomenology, prevalence, and comorbidity. Child and Adolescent Psychiatric Clinics of North America, 14, 631-648. doi:10.1016/j.chc.2005.06.003

Cotton, S. P., Levine, E. G., Fitzpatrick, C. M., Dold, K. H., \& Targ, E. (1999). Exploring the relationships among spiritual wellbeing, quality of life and psychological adjustment in women with breast cancer. Psycho-Oncology, 8, 429-438.

Cowie, J., Clementi, M. A., \& Alfano, C. A. (2016). Examination of the Intolerance of 
Uncertainty Construct in Youth With Generalized Anxiety Disorder. Journal of Clinical Child \& Adolescent Psychology, 1-9.

Creveling, C. C., Varela, R. E., Weems, C. F., \& Corey, D. M. (2010). Maternal control, cognitive style, and childhood anxiety: A test of a theoretical model in a multiethnic sample. Journal of Family Psychology, 24, 439-448. doi:10.1037/a0020388

Cuéllar, I., Arnold, B., \& González, G. (1995) Cognitive referents of acculturation: Assessment of cultural constructs in Mexican Americans. Journal of Community Psychology, 23, 339-356.

Dalton, K. M., Kalin, N. H., Grist, T. M., \& Davidson, R. J. (2005). Neural-Cardiac Coupling in Threat-Evoked Anxiety. Journal of Cognitive Neuroscience, 17, 969980. doi:10.1162/0898929054021094

Derogatis, L. R. (2001). Brief Symptom Inventory 18: Administration scoring and procedures manual. Minneapolis: National Computer Systems.

Diaz, D., Blanco, A., Bajo, M. \& Stavraki, M. (2015). Fatalism and well-being across Hispanic cultures: The social fatalism scales (SFS). Soc Indic Res, 124, 929-945. doi:10.1007/s11205-014-0825-1

Dugas, M. J., Gagnon, F., Ladouceur, R., \& Freeston, M. H. (1998). Generalized anxiety disorder: A preliminary test of a conceptual model. Behaviour Research and Therapy, 36, 215-226. doi:10.1016/S0005-7967(97)00070-3

Dugas, M. J., \& Robichaud, M. (2007). Cognitive-behavioral treatment for generalized anxiety disorder: From science to practice. New York: Routledge.

Durkheim, D. E. (1897). Le suicide: Étude de sociologie. Paris

Elkind, D. (1967). Egocentrism in adolescence. Child Development, 38, 1025-1034. 
Esparza, O. A., Wiebe, J. S., \& Quiñones, J. (2015). Simultaneous development of a multidimensional fatalism measure in English and Spanish. Current Psychology: A Journal for Diverse Perspectives on Diverse Psychological Issues, 34, 597-612. doi:10.1007/s12144-014-9272-z

Espinosa de los Monteros, K., \& Gallo, L. (2011). The relevance of fatalism in the study of Latinas' cancer screening behavior: A systematic review of the literature. International Journal of Behavioral Medicine, 18, 310-318.

Futa, K. T., Hsu, E., \& Hansen, D. J. (2001). Child sexual abuse in Asian American families: An examination of cultural factors that influence prevalence, identification, and treatment. Clinical Psychology: Science and Practice, 8, 189209. doi:10.1093/clipsy.8.2.189.

Gallagher, M. W., Bentley, K. H., \& Barlow, D. H. (2014). Perceived control and vulnerability to anxiety disorders: A meta-analytic review. Cognitive Therapy and Research, 38, 571-584. doi:10.1007/s10608-014-9624-x

Gazelle, H., \& Ladd, G. W. (2003). Anxious solitude and peer exclusion: A diathesisstress model of internalizing trajectories in childhood. Child Development, 74, 257-278. doi:10.1111/1467-8624.00534

Gelman, A., \& Hill, J. (2006). Missing-data imputation. In Data Analysis Using Regression and Multilevel/Hierarchical Models (Analytical Methods for Social Research, pp. 529-544). Cambridge: Cambridge University Press. doi:10.1017/CBO9780511790942.031

Gil, R. M., \& Vasquez, C. I. (1996). The Maria paradox. New York: G.P. Putnam. 
Giollabhui, N. M., Nielsen, J., Seidman, S., Olino, T. M., Abramson, L. Y., \& Alloy, L. B. (2018). The development of future orientation is associated with faster decline in hopelessness during adolescence. Journal of Youth and Adolescence, 47, 2129 2142. doi: 10.1007/s10964-017-0803-4

Glover, S. H., Pumariega, A. J., Holzer, C. E., III., Wise, B. K., \& Rodriguez, M. (1999). Anxiety symptomatology in Mexican-American adolescents. Journal of Child and Family Studies, 8, 47-57. doi:10.1023/A:1022994510944

Greenwell, A. N., \& Cosden, M. (2009). The relationship between fatalism, dissociation, and trauma symptoms in Latinos. Journal of Trauma \& Dissociation, 10, 334345.

Greer, S., Morris, T. \& Pettingale, K. (1979). Psychological response to breast cancer: effect on outcome. The Lancet, 314, 785-787.

Guzmán, M. R., Santiago-Rivera, A. L., \& Hasse, R. F. (2005). Understanding Academic Attitudes and Achievement in Mexican-Origin Youths: Ethnic Identity, OtherGroup Orientation, and Fatalism. Cultural Diversity and Ethnic Minority Psychology, 11, 3-15. doi:10.1037/1099-9809.11.1.3

Haack, L. M, Gerdes, A. C., \& Lawton, K. E. (2014). Conducting research with Latino families: Examination of strategies to improve recruitment, retention, and satisfaction with an at-risk and underserved population. Journal of Child and Family Studies, 23, 410-421. doi:10.1007/s10826-012-9689-7

Hacker, K., Chu, J., Leung, C., Marra, R., Pirie, A., Brahimi, M., \& ... Marlin, R. P. (2011). The impact of immigration and customs enforcement on immigrant 
health: Perceptions of immigrants in Everett, Massachusetts, USA. Social Science \& Medicine, 73, 586-594. doi:10.1016/j.socscimed.2011.06.007

Hayes, A. (2013). Introduction to mediation, moderation, and conditional process analysis. New York, NY: Guilford, 3-4. doi: 978-1-60918-230-4

Hayes, S. C., Strosahl, K. D., \& Wilson, K. G. (1999). Acceptance and commitment therapy: An experiential approach to behavior change. New York, NY: Guilford Press.

Helzer, E. G., \& Jayawickreme, E. (2015). Control and the 'good life': Primary and secondary control as distinct indicators of well-being. Social Psychological and Personality Science, 6, 653-660. doi:10.1177/1948550615576210

Herrera, C. J., Owens, G. P., \& Mallinckrodt, B. (2013). Traditional machismo and caballerismo as correlates of posttraumatic stress disorder, psychological distress, and relationship satisfaction in Hispanic veterans. Journal of Multicultural Counseling and Development, 41, 21-35. doi:10.1002/j.2161-1912.2013.00024.x

Hirschman, E. C. (2003). Men, dogs, guns, and cars: The semiotics of rugged individualism. Journal of Advertising, 32, 9-22.

Ho, M.K. (1987). Family therapy with ethnic minorities. Newbury, CA: Sage.

Hubbell, F. A., Chavez, L. R., Mishra, S. I., \& Valdez, R. B. (1996). Differing beliefs about breast cancer among Latinas and Anglo women. The Western Journal of Medicine, 164, 405-409.

Joiner, T. E., Perez, M., Wagner, K. D., Berenson, A., \& Marquina, G. S. (2001). On fatalism, pessimism, and depressive symptoms among Mexican-American and 
other adolescents attending an obstetrics-gynecology clinic. Behavior Research and Therapy, 39, 887-896. doi:10.1016/S0005-7967(00)00062-0.

Kapke, T. L., Grace, M. A., Gerdes, A. C., \& Lawton, K. E. (2017). Latino early adolescent mental health: Examining the impact of family functioning, familism, and global self-worth. Journal of Latina/o Psychology, 5, 27-44. doi:10.1037/lat0000057

Keough, M. E., Timpano, K. R., \& Schmidt, N. B. (2009). Ataques de nervios: Culturally bound and distinct from panic attacks?. Depression and Anxiety, 26, 16-21. doi:10.1002/da.20498

King N. J., Ollendick T. H., \& Gullone E. (1991). Negative affectivity in children and adolescents: relations between anxiety and depression. Clinical Psychology Review, 11, 441-459.

LaFromboise, T., Coleman, H. L. K., \& Gerton, J. (1993). Psychological impact of biculturalism: Evidence and theory. Psychological Bulletin, 114, 395-412. doi:10.1037/0033-2909.114.3.395

Lewinsohn, P. M., Gotlib, I. H., Lewinsohn, M., Seeley, J. R., \& Allen, N. B. (1998). Gender differences in anxiety disorders and anxiety symptoms in adolescents. Journal of Abnormal Psychology, 107, 109-117. doi:10.1037/0021843X.107.1.109

Lewis-Fernández, R., Guarnaccia, P. J., Martínez, I. E., Salmán, E., Schmidt, A., \& Liebowitz, M. (2002). Comparative phenomenology of ataques de nervios, panic attacks, and panic disorder. Culture, Medicine and Psychiatry, 26, 199-223. doi:10.1023/A:1016349624867 
Little, R. J. A. (1988). A test of missing completely at random for multivariate data with missing values. Journal of the American Statistical Association, 83, 1198-1202. doi:10.1080/01621459.1988.10478722

Luis, T. M., Varela, R. E., \& Moore, K. W. (2008). Parenting practices and childhood anxiety reporting in Mexican, Mexican American, and White, non-Latino families. Journal of Anxiety Disorders, 22, 1011-1020. doi:10.1016/j.janxdis.2007.11.001

Maercker, A., Ben-Ezra, M., Esparza, O. A., \& Augsburger, M. (2019). Fatalism as a traditional cultural belief potentially relevant to trauma sequelae: Measurement equivalence, extent and associations in six countries. European Journal of Psychotraumatology, 10, 1-13. doi:10.1080/20008198.2019.1657371

Magai, C., Consedine, N., Neugut, A. I., \& Hershman, D. L. (2007). Common psychosocial factors underlying breast cancer screening and breast cancer treatment adherence: A conceptual review and synthesis. Journal of Women's Health, 16, 11-23. doi:10.1089/jwh.2006.0024

Magaro, M. M., \& Weisz, J. R. (2006). Perceived Control Mediates the Relation Between Parental Rejection and Youth Depression. Journal of Abnormal Child Psychology, 34, 867-876. doi:10.1007/s10802-006-9072-5

Marin, G., \& VanOss Marin, B. (1991). Research with Hispanic populations. Newbury Park, CA: Sage.

Martinez, C. R., Schwartz, S. J., Thier, M., \& McClure, H. H. (2018). A tale of two measures: Concordance between the ARSMA-II and the BIQ acculturation scales 
among Latino immigrant families. Psychological Assessment, 30, 459-473. doi: 10.1037/pas0000491

Masuda, A., Ng, S. Y., Moore, M., Felix, I., \& Drake, C. E. (2016). Acceptance and commitment therapy as a treatment for a Latina young adult woman with purging: A case report. Practice Innovations, 1, 20-35. doi: 10.1037/pri0000012

McCaul, K. D., Solomon, S., \& Holmes, D. S. (1979). Effects of paced respiration and expectations on physiological and psychological responses to threat. Journal of Personality and Social Psychology, 37, 564-571. doi: 10.1037/00223514.37.4.564

McLean, C. P. \& Anderson, E. R. (2009) Brave men and timid women? A review of the gender differences in fear and anxiety. Clinical Psychology Review, 29, 496-505.

McLean, C. P., Asnaani, A., Litz, B. T., \& G, H. S. (2011). Gender differences in anxiety disorders: Prevalence, course of illness, comorbidity and burden of illness. Journal of Psychiatric Research, 45, 1027-1035. doi:10.1016/j.jpsychires.2011.03.006

Meyer, T. J., Miller, M. L., Metzger, R. L., \& Borkovec, T. D. (1990). Development and validation of the Penn State Worry Questionnaire. Behavior Research and Therapy, 28, 487-495.

Moorey, S. and Greer, S. (1989) Psychological Therapy for Patients with Cancer: A New Approach. American Psychiatric Press, Washington DC.

Morling, B., \& Fiske, S. T. (1999) Defining and measuring harmony control. Journal of Research in Personality, 33, 379-414. 
Muris, P. (2006). Maladaptive schemas in non-clinical adolescents: Relations to perceived parental rearing behaviors, big five personality factors and psychopathological symptoms. Clinical Psychology and Psychotherapy, 13, 405413. doi:10.1002/cpp.506

Navarro, R., Yubero, S., \& Larrañaga, E. (2018) Cyberbullying victimization and fatalism in adolescence: Resilience as a moderator. Children and Youth Services Review, 84, 215-221. doi:10.1016/j.childyouth.2017.12.011

Neff, J. A., \& Hoppe, S. K. (1993). Race/ethnicity, acculturation, and psychological distress: Fatalism and religiosity as cultural resources. Journal of Community Psychology, 21, 3-20.

Norton, P. J. (2005). A psychometric analysis of the Intolerance of Uncertainty Scale among four racial groups. Journal of Anxiety Disorders, 19, 699-707.

Novy, D. M., Stanley, M. A., Averill, P., \& Daza, P. (2001). Psychometric comparability of English- and Spanish-language measures of anxiety and related affective symptoms. Psychological Assessment, 13, 347-355. doi:10.1037/10403590.13 .3 .347

Nurmi, J. -E. (1991). How do adolescents see their future? A review of the development of future orientation and planning. Developmental Review, 11, 1-59. doi:10.1016/0273-2297(91)90002-6

Palloni, A., \& Arias, E. (2004). Paradox lost: explaining the Hispanic adult mortality advantage. Demography, 41, 385-415.

Paranjape, A., \& Kaslow, N. (2010). Family violence exposure and health outcomes among older African American women: Does spirituality and social support play 
a protective role?. Journal of Women's Health, 19, 1899-1904.

doi:10.1089/jwh.2009.1845

Parker, S., \& Kleiner, R. (1966). Mental illness in the urban Negro community. New York: Free Press.

Pennebaker, J. W., Chung, C. K., Ireland, M., Gonzales, A., \& Booth, R. J. (2007). The development and psychometric properties of LIWC2007. Austin, TX: LIWC.

Pestle, S. L., Chorpita, B. F., \& Schiffman, J. (2008). Psychometric properties of the Penn State worry questionnaire for children in a large clinical sample. Journal of Clinical Child and Adolescent Psychology, 37, 465-471.

doi:10.1080/15374410801955896

Peterson, R. A., \& Reiss, S. (1993). Anxiety Sensitivity Index Revised test manual. Worthington, OH: IDS Publishing Corporation.

Pettingale, D., Morris, T., Greer, S. \& Haybittle, J. (1985) Mental attitudes to cancer: an additional prognostic factor. Lancet, 1, 750.

Pew Research Center (2017). Latinos and the New Trump Administration. Retrieved from http://www.pewhispanic.org/2017/02/23/latinos-and-the-new-trumpadministration/

Piña-Watson, B., \& Abraído-Lanza, A. F. (2017). The intersection of fatalism and pessimism on depressive symptoms and suicidality of Mexican descent adolescents: An attribution perspective. Cultural Diversity and Ethnic Minority Psychology, 23, 91-101. 
Piña-Watson, B., Castillo, L. G., Jung, E., Ojeda, L., \& Castillo-Reyes, R. (2014). The Marianismo Beliefs Scale: Validation with Mexican American adolescent girls and boys. Journal of Latina/o Psychology, 2, 113-130. doi: 10.1037/lat0000017

Powe, B. D. (1995). Fatalism among elderly African Americans: Effects on colorectal screening. Cancer Nursing, 18, 385-392.

Radloff, L. S. (1977). The CES-D scale: a self-report depression scale for research in the general population. Applied Psychological Measurement, 1, 385-401.

Reynolds, C. R., \& Richmond, B. O. (1997). Revised Children's Manifest Anxiety Scale

Manual. Journal of Abnormal Child Psychology, 6, 271-280. doi:10.1037/t00514

Roberts, R. E., Roberts, C. R., \& Chen, I. G. (2000). Fatalism and risk of adolescent depression. Psychiatry: Interpersonal and Biological Processes, 63, 239-252.

Roberts, R. E., Roberts, C. R., \& Chen, Y. R. (1998). Suicidal thinking among adolescents with a history of attempted suicide. Journal of the American Academy of Child \& Adolescent Psychiatry, 37, 1294-1300. doi:10.1097/00004583199812000-00013

Romero-Acosta, K., Penelo, E., Noorian, Z., Ferreira, E., \& Domènech-Llaberia, E. (2014). Racial/ethnic differences in the prevalence of internalizing symptoms: Do Latin-American immigrant show more symptomatology than Spanish native-born adolescents? Journal of Health Psychology, 19, 381-392. doi:10.1177/1359105312471568

Roncancio, A. M., Ward, K. K., \& Berenson, A. B. (2011). Hispanic women's health care provider control expectations: The influence of fatalism and 
acculturation. Journal of Health Care for the Poor and Underserved, 22, 482-490. doi:10.1353/hpu.2011.0038

Rosales, R. \& Calvo, R. (2017). "Si Dios quiere": Fatalismo and use of mental health services among Latinos with a history of depression. Social Work in Health Care, 56, 748-764. doi: 10.1080/00981389.2017.1339760

Ross, C. E., Mirowsky, J., \& Cockerham, W. C. (1983). Social class, Mexican culture, and fatalism: Their effects on psychological distress. American Journal of Community Psychology, 11, 383-399. doi:10.1007/BF00894055.

Rubalcava, L. N., Teruel, G. M., Thomas, D., \& Goldman, N. (2008). The healthy migrant effect: New findings from the Mexican Family Life Survey. American Journal of Public Health, 98, 78-84. doi:10.2105/AJPH.2006.098418

Ruiz, J. M., Hamann, H. A., Mehl, M. R., \& O’Connor, M. F. (2016). The Hispanic health paradox: From epidemiological phenomenon to contribution opportunities for psychological science. Group Processes \& Intergroup Relations, 19, 462-476.

Scheier, M. F., \& Bridges, M. W. (1995). Person variables and health: Personality predispositions and acute psychological states as shared determinants for disease. Psychosomatic Medicine, 57, 255-268.

Schwartz, S. J., Unger, J. B., Lorenzo-Blanco, E. I., Des Rosiers, S. E., Villamar, J. A., Soto, D. W., ... Szapocznik, J. (2014). Perceived context of reception among recent Hispanic immigrants: Conceptualization, instrument development, and preliminary validation. Cultural Diversity and Ethnic Minority Psychology, 20, 115. doi: $10.1037 / \mathrm{a} 0033391$ 
Schwartz, S. J., Unger, J. B., Zamboanga, B. L., Córdova, D., Mason, C. A., Huang, S., ... Szapocznik, J. (2015). Developmental Trajectories of Acculturation: Links with Family Functioning and Mental Health in Recent-Immigrant Hispanic Adolescents. Child Development, 86, 726-748. doi: 10.1111/cdev.12341

Schwartz, S. J., Unger, J. B., Zamboanga, B. L., \& Szapocznik, J. (2010). Rethinking the concept of acculturation: Implications for theory and research. American Psychologist, 65, 237-251. doi:10.1037/a0019330.

Schwartz, S. J., Weisskirch, R. S., Zamboanga, B. L., Castillo, L. G., Ham, L. S., Huynh, Q. -L., . . Cano, M. A. (2011). Dimensions of acculturation: Associations with health risk behaviors among college students from immigrant families. Journal of Counseling Psychology, 58, 27-41. doi:10.1037/a0021356

Sharrief, A. Z., Sánchez, B. N., Lisabeth, L. D., Skolarus, L. E., Zahuranec, D. B., Baek, J., ... Morgenstern, L. B. (2017). The impact of pre-stroke depressive symptoms, fatalism, and social support on disability after stroke. Journal of Stroke and Cerebrovascular Diseases, 26, 2686-2691.

Smokowski, P., Buchanan, R. L., \& Bacallao, M. L. (2009). Acculturation and adjustment in Latino adolescents: How cultural risk factors and assets influence multiple domains of adolescent mental health. Journal of Primary Prevention, 30 , 371-393. doi: 10.1007/s10935-009-0179-7

Soto, C., Unger, J. B., Ritt-Olson, A., Soto, D. W., Black, D. S., \& Baezconde-Garbanati, L. (2011). Cultural values associated with substance use among Hispanic adolescents in Southern California. Substance Use \& Misuse, 46, 1223-1233. doi:10.3109/10826084.2011.567366 
Spielberger, C. D., Jacobs, G. A., Crane, R. S., Russell, S. F., Westberry, L., Barker, L., ... Marks, E. (1979). State-Trait Personality Inventory. Tampa: Human Resources Institute, University of South Florida.

Spurlock, W. R., \& Cullins, L. S. (2006). Cancer fatalism and breast cancer screening in African American women. ABNF J, 17, 38-43.

Steer, R. A., Kumar, G., Beck, J. S., \& Beck, A. T. (2001). Evidence for the construct validities of the Beck Youth Inventories with child psychiatric outpatients. Psychological Reports, 89, 559-565. doi:10.2466/PR0.89.7.559-565

Steiner H., Ryst E., Berkowitz J., Gschwendt M. A., \& Koopman C. (2002). Boys’and girls' responses to stress: affect and heart rate during a speech task. Journal of Adolescent Health, 30, 14-21.

Szapocznik, J., Kurtines, W. M., \& Fernandez, T. (1980). Bicultural involvement and adjust in Hispanic-American youths. International Journal of Intercultural Relations, 4, 353-365.

Teruya, S. A., \& Bazargan-Hejazi, S. (2013). The immigrant and Hispanic paradoxes: A systematic review of their predictions and effects. Hispanic Journal of Behavioral Sciences, 35, 486-509. doi:10.1177/0739986313499004

Umaña-Taylor, A. J., Updegraff, K. A., and Gonzales-Backen, M. A. (2011). Mexicanorigin adolescent mothers' stressors and psychosocial functioning: examining ethnic identity affirmation and familism as moderators. Journal of Youth and Adolescence, 40, 140-157. doi: 10.1007/s10964-010- 9511-z

U.S. Census Bureau (2016). ACS Demographic and Housing Estimates, 20122016 American Community Survey 5-year Estimates. Retrieved from 
https://factfinder.census.gov/faces/tableservices/jsf/pages/productview.xhtml?pid =ACS_16_5YR_DP05\&src=pt.

Valdivieso-Mora, E., Peet, C. L., Garnier-Villarreal, M., Salazar-Villanea, M., \& Johnson, D. K. (2016). A systematic review of the relationship between familism and mental health outcomes in Latino population. Frontiers In Psychology, 7, 113.

Varela, R. E., Sanchez-Sosa, J. J., Biggs, B. K., \& Luis, T. M. (2008). Anxiety symptoms and fears in Latin American and European American children: Cross-cultural measurement equivalence. Journal of Psychopathology and Behavioral Assessment, 30, 132-145. doi:10.1007/s10862-007-9056-y

Varela, R. E., Vernberg, E. M., Sanchez-Sosa, J. J., Riveros, A., Mitchell, M., \& Mashunkashey, J. (2004). Anxiety reporting and culturally associated interpretation biases and cognitive schemas: A comparison of Mexican, Mexican American, and European American families. Journal of Clinical Child and Adolescent Psychology, 33, 237-247. doi:10.1207/s15374424 jccp3302_4

Velasco-Mondragon, E., Jimenez, A., Palladino-Davis, A. G., Davis, D., \& EscamillaCejudo, J. A. (2016). Hispanic health in the USA: A scoping review of the literature. Public Health Reviews, 37. doi: 10.1186/s40985-016-0043-2

Waite, P., Codd., J. \& Creswell, C. (2015). Interpretation of ambiguity: Differences between children and adolescents with and without an anxiety disorder. Journal of Affective Disorders, 188, 194-201. doi: 10.1016/j.jad.2015.08.022 
Walker, R. J., Smalls, B. L., Hernandez-Tejada, M. A., Campbell, J. A., Davis, K. S., \& Egede, L. E. (2012). Effect of diabetes fatalism on medication adherence and selfcare behaviors in adults with diabetes. General Hospital Psychiatry, 34, 598-603.

Waschbusch, D. A., Sellers, D. P., LeBlanc, M., \& Kelley, M. L. (2003). Helpless attributions and depression in adolescents: The role of anxiety, event valence, and demographics. Journal of Adolescence, 26, 169-183. doi: 10.1016/S01401971(02)00134-3

Watson, M., Law, M., dos Santos, M., Greer, S., Baruch, J. \& Bliss, J. M. (1994). The Mini-MAC: Further development of the Mental Adjustment to Cancer Scale. Journal of Psychosocial Oncology, 12, 33-46.

Weisz, J. R., Francis, S. E., \& Bearman, S. K. (2010). Assessing secondary control and its association with youth depression symptoms. Journal of Abnormal Child Psychology, 38, 883-893.

Weisz, J. R., Rothbaum, F. M., \& Blackburn, T. C. (1984) Standing out and standing in: The psychology of control in American and Japan. American Psychologist, 39, 955-969.

Weisz, J. R., Southam-Gerow, M. A., \& Sweeney, L. (1998). The Perceived Control Scale for Children. Los Angeles: University of California, Los Angeles

Weller, S. C., Baer, R. D., de Alba, J. G., Glazer, M., Trotter, R., Pachter, L., \& Klein, R. E. (2002). Regional variation in Latino beliefs about susto. Culture, Medicine, and Psychiatry, 26, 449-472.

Wells, A. (1999). A metacognitive model and therapy for generalized anxiety disorder. Clinical Psychology \& Psychotherapy, 6, 86-95. 
Wood, J. J., McLeod, B. D., Sigman, M., Hwang, W. C., \& Chu, B. C. (2003). Parenting and childhood anxiety: Theory, empirical findings, and future directions. Journal of Child Psychology and Psychiatry, 44, 134-151. doi:10.1111/1469-7610.00106

Zeiders, K. H., Updegraff, K. A., Umaña-Taylor, A. J., Wheeler, L. A., Perez-Brena, N. J., \& Rodríguez, S. A. (2013). Mexican-origin youths' trajectories of depressive symptoms: the role of familism values. Journal of Adolescent Health, 53, 648654. doi: 10.1016/j.jadohealth.2013.06.008 


\section{Appendix}

\section{Appendix A. Modified Story Completion Task}

1. Alisa enters math class and is told there will be a surprise quiz today.

a. What happens next?

b. How much do you agree with the following statement? Rate from 1 "strongly disagree" to 5 "strongly agree"

What happens is determined by fate

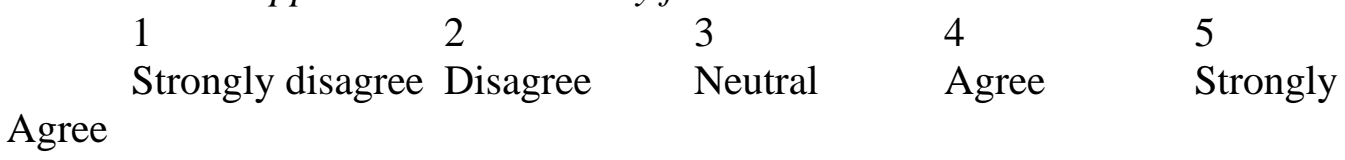

c. How worried do you think Alisa is? Rate from 1 "not at all" to 5 "very, very worried"

$\begin{array}{lllll} & 2 & 3 & 4 & 5 \\ \text { Not at all } & \text { A little } & \text { Some } & \text { A lot } & \text { Very, very }\end{array}$

worried

Not at all A little $\quad$ Some $\quad$ A lot $\quad$ Very, very

2. Hector's soccer team is playing a playoff game this weekend against a really good team.

a. What happens next?

b. How much do you agree with the following statement? Rate from 1 "strongly disagree" to 5 "strongly agree"

Hector must live for today, who knows what the future will bring

$\begin{array}{lllll}1 & 2 & 3 & 4 & 5 \\ \text { Strongly disagree } & \text { Disagree } & \text { Neutral } & \text { Agree } & \text { Strongly }\end{array}$

Agree

c. How worried do you think Hector is? Rate from 1 "not at all" to 5 "very, very worried"

$\begin{array}{lllll}1 & 2 & 3 & 4 & 5 \\ \begin{array}{l}\text { Not at all } \\ \text { rried }\end{array} & \text { A little } & \text { Some } & \text { A lot } & \text { Very, very }\end{array}$

3. Melissa would like to go to prom, but she does not know if anyone will ask her to go with them.

a. What happens next? 
b. How much do you agree with the following statement? Rate from 1 "strongly disagree" to 5 "strongly agree"

No matter what Melissa does, what is meant to happen will happen anyway Agree

$\begin{array}{lllll}1 & 2 & 3 & 4 & 5 \\ \text { Strongly disagree } & \text { Disagree } & \text { Neutral } & \text { Agree } & \text { Strongly }\end{array}$

c. How worried do you think Melissa is? Rate from 1 "not at all" to 5 "very, very worried"

$\begin{array}{lllll}1 & 2 & 3 & 4 & 5 \\ \text { Not at all } & \text { A little } & \text { Some } & \text { A lot } & \text { Very, very }\end{array}$
worried

4. Marco went to the doctor for a regular check-up. He was sent to do x-rays and told he will get the results in two weeks.

a. What happens next?

b. How much do you agree with the following statement? Rate from 1 "strongly disagree" to 5 "strongly agree"

Marco's life is determined by something greater than himself

$\begin{array}{lllll}1 & 2 & 3 & 4 & 5 \\ \text { Strongly disagree } & \text { Disagree } & \text { Neutral } & \text { Agree } & \text { Strongly }\end{array}$

Agree

c. How worried do you think Marco is? Rate from 1 "not at all" to 5 "very, very worried"

1

Not at all worried

$2 \quad 3$

A little
3

Some
4

A lot
5

Very, very

5. Julia auditioned to be in a school play and was competing against many other students. She has not yet heard back on whether she got the part in the play.

a. What happens next?

b. How much do you agree with the following statement? Rate from 1 "strongly disagree" to 5 "strongly agree"

What happens is a matter of luck

$\begin{array}{lllll}1 & 2 & 3 & 4 & 5 \\ \text { Strongly disagree } & \text { Disagree } & \text { Neutral } & \text { Agree } & \text { Strongly }\end{array}$


c. How worried do you think Julia is? Rate from 1 "not at all" to 5 "very, very worried"

$\begin{array}{lllll}1 & 2 & 3 & 4 & 5 \\ \text { Not at all } & \text { A little } & \text { Some } & \text { A lot } & \text { Very, very }\end{array}$
worried

6. Jonathan is planning to ask a new friend to come to his birthday party, but Jonathan is not sure if they will say yes.

a. What happens next?

b. How much do you agree with the following statement? Rate from 1 "strongly disagree" to 5 "strongly agree"

There is not much that can be done about this situation

$\begin{array}{lllll}1 & 2 & 3 & 4 & 5 \\ \text { Strongly disagree } & \text { Disagree } & \text { Neutral } & \text { Agree } & \text { Strongly }\end{array}$
Agree

c. How worried do you think Jonathan is? Rate from 1 "not at all" to 5 "very, very worried"

$\begin{array}{lllll}1 & 2 & 3 & 4 & 5 \\ \begin{array}{l}\text { Not at all } \\ \text { rried }\end{array} & \text { A little } & \text { Some } & \text { A lot } & \text { Very, very }\end{array}$

7. Sam entered a radio contest to see their favorite singer in concert.

a. What happens next?

b. How much do you agree with the following statement? Rate from 1 "strongly disagree" to 5 "strongly agree"

What happens is in the hands of God

$\begin{array}{lllll}1 & 2 & 3 & 4 & 5 \\ \text { Strongly disagree } & \text { Disagree } & \text { Neutral } & \text { Agree } & \text { Strongly } \\ \text { Agree } & & & & \end{array}$

c. How worried do you think Sam is? Rate from 1 "not at all" to 5 "very, very worried"

1

Not at all worried
$2 \quad 3$

A little Some
4

A lot
5

Very, very

8. Elena wants to be an engineer when she grows up but is unsure of how to become an engineer. 
a. What happens next?

b. How much do you agree with the following statement? Rate from 1 "strongly disagree" to 5 "strongly agree"

What happens is determined by fate

$1 \quad 2 \quad 3 \quad 4 \quad 5$

Agree

Strongly disagree Disagree Neutral Agree Strongly

c. How worried do you think Elena is? Rate from 1 "not at all" to 5 "very, very worried"

$\begin{array}{lllll}1 & 2 & 3 & 4 & 5 \\ \text { Not at all } & \text { A little } & \text { Some } & \text { A lot } & \text { Very, very }\end{array}$
worried

9. Berto is starting at a new school next month. He thinks about what it will be like.

a. What happens next?

b. How much do you agree with the following statement? Rate from 1 "strongly disagree" to 5 "strongly agree"

It is more important to enjoy life now than to plan for the future

$\begin{array}{lllll}1 & 2 & 3 & 4 & 5 \\ \text { Strongly disagree } & \text { Disagree } & \text { Neutral } & \text { Agree } & \text { Strongly }\end{array}$
Agree

c. How worried do you think Berto is? Rate from 1 "not at all" to 5 "very, very worried"

$\begin{array}{lllll}1 & 2 & 3 & 4 & 5 \\ \text { Not at all } & \text { A little } & \text { Some } & \text { A lot } & \text { Very, very }\end{array}$ worried

10. Rosa's sister is having her baby shower at the park this weekend. The weather forecast says there is a chance of rain.

a. What happens next?

b. How much do you agree with the following statement? Rate from 1 "strongly disagree" to 5 "strongly agree"

If something bad is meant to happen, it will happen

$\begin{array}{lllll}1 & 2 & 3 & 4 & 5 \\ \text { Strongly disagree } & \text { Disagree } & \text { Neutral } & \text { Agree } & \text { Strongly }\end{array}$

Agree 
c. How worried do you think Rosa is? Rate from 1 "not at all" to 5 "very, very worried"

$\begin{array}{lllll}1 & 2 & 3 & 4 & 5 \\ \text { Not at all } & \text { A little } & \text { Some } & \text { A lot } & \text { Very, very }\end{array}$
worried 


\section{CURRICULUM VITAE}

\section{Judy Mier-Chairez, M.S.}

1515 Demonbreun St.| Nashville, TN 78202

Tel: (616) 648-3193| E-mail: mierjudy@gmail.com

\section{EDUCATION}

Pre-Doctoral Intern, CBT Track

University of Texas Health San Antonio-San Antonio, TX

expected: 2020

Site: Family and Community Medicine

Site Supervisor: Stacy Ogbeide, PsyD, ABPP

Preceptor: Kathryn Kanzler, PsyD, ABPP

Doctoral Student (Ph.D.), Clinical Psychology

expected: 2020

University of Louisville- Louisville, $\mathrm{KY}$

Advisor: Janet Woodruff-Borden, $\mathrm{PhD}$

Dissertation: Fatalism as a Cultural Influence on Correlates of Anxiety and Worry in Latino/a Adolescents

Masters of Science (M.S.), Clinical Psychology

University of Louisville- Louisville, KY

Advisors: Monnica Williams, PhD, Janet Woodruff-Borden, PhD

Bachelor of Arts (B.A.), Psychology major

University of Colorado- Boulder, CO

Cumulative Grade Point Average: 3.89

\section{AWARDS AND HONORS}

Clinical Psychology Department Award for Excellence in Clinical Practice

Clinical Psychology Department Award for Excellence in Professional Service

Stanley A. Murrell Scientist-Practitioner Award-nominated

Graduate Student Council Research Fund, \$500

Fatalism as a Cultural Influence on Correlates of Anxiety and Worry in Latino/a Adolescents

Preliminary Qualifying Examination: Distinguished Pass 2018

Graduate Network in Arts and Sciences Research Fund, \$200 2018

Investigating the Role of Fatalism in Anxiety and Worry in the Latino/a Population

McSweeney Diversity Research Fellowship

2017

Graduate Student Council Conference Travel Award, \$350

2016 
Graduated with Distinction (B.A.)

2013

Phi Beta Kappa Honors Society member

2013

\section{CLINICAL EXPERIENCE}

Behavioral Health Consultation

Department of Family and Community Medicine- Primary Care Behavioral Health

Integration

July 2019-Present

Supervisor: Stacy Ogbeide, Psy.D., ABPP

- Provide behavioral health interventions in English and Spanish as part of the primary care medical team in the form of warm hand-offs and scheduled consultations

- Consultation with physicians, nurse practitioners, physician assistants, social workers, nurses, medical assistants and other medical staff to coordinate patient care

- Supervise Family Medicine residents and medical students learning the primary care behavioral health model

- Behavioral medicine interventions including weight management, chronic pain management, tobacco cessation, treatment adherence, diabetes management, sleep interventions

- Daily supervision using the precepting model

- Weekly supervision on provision of supervision

- Treatment modalities: brief cognitive-behavioral therapy, focused acceptance and commitment therapy, motivational interviewing, mindfulness-based interventions

Dialectical Behavior Therapy Skills Group

UT Health San Antonio Department of Psychiatry, San Antonio, TX

July 2019-Present

Supervisor: Lisa Smith Kilpela, Ph.D

- Co-lead weekly skills group for patients referred by their individual providers

- Prepare lesson plan for teaching skills modules

- Teach skills modules including didactics, personal examples, and skills practice

- Teaching Mindfulness, Emotion Regulation, Distress Tolerance, and Interpersonal Effectiveness skills

- Manage disruptive behaviors in real-time and provide crisis intervention

- Attend weekly DBT consultation group

- Live supervision from DBT expert

Advance Clinic

UT Health San Antonio Department of Psychiatry, San Antonio, TX

July 2019-Present

Supervisor: Paul Nabity, Ph.D.

- Provide individual psychotherapy for patients within the Department of Psychiatry

- Weekly individual supervision including audio/digital recording review

- Treatment modalities: cognitive processing therapy, cognitive-behavioral therapy, acceptance and commitment therapy, problem-solving therapy

Integrated Interventions Treatment Team 
Noble H. Kelley Psychological Services Center, Louisville, KY

August 2017-June 2019

Supervisor: Richard Lewine, Ph.D.

- Conduct therapy intake evaluations for adults and adolescents with a variety of psychological disorders including serious mental illness, mood disorders, anxiety disorders, and trauma- and stressor-related disorders in a diverse client population

- Implement an integrated, person-centered approach to therapy via empirically supported methods

- Formulate client conceptualizations and tailored treatment plans with reference to relevant empirical literature and implementation of disorder-specific manualized treatments as appropriate

- Weekly group and individual supervision, peer consultation, audio/digital recording and live observations, case conceptualization

- Monthly supervision on provision of peer supervision

- Treatment modalities: cognitive-behavioral, mindfulness-based, dialectical-behavioral, interpersonal, psychodynamic

\section{Pediatric Primary Care Psychological Services}

University of Louisville Pediatrics, Louisville, KY

July 2018-June 2019

Supervisor: Bernadette Walter, Ph.D.

- Provide consultation for physicians on their primary care patients

- Provide brief interventions in English and Spanish for children and adolescents ages 3-17 and their parents for a variety of concerns including trauma- and stress-related symptoms, anxiety disorders, behavior and conduct difficulties, weight management, and post-partum depression

- Received weekly individual supervision

Assessment and Testing Practicum Noble H. Kelley Psychological Services Center, Louisville, KY August 2016-June 2019

Supervisors: David Winsch, Ph.D. and Bernadette Walter, Ph.D.

- Conduct and score psychological and neuropsychological assessment batteries for children and adults ages 11-69 for concerns including ADHD, learning disorders, dementia, placement in gifted and talented programs

- Administer semi-structured interviewing for diagnostic assessments with adults, children and adolescents, and parents of children and adolescents

- Consult with health providers and teachers for diagnostic purposes

- Prepare integrative reports

- Conduct feedback sessions

- Assessment tools utilized: WAIS-IV, WISC-V, WIAT-III, WJ-III, WRAT-4, MMPI, MACI, CPT, ADIS-5 Client, ADIS-IV Parent and Child, Bender Gestalt Test

Clinical Graduate Teaching Assistant

Noble H. Kelley Psychological Services Center, Louisville, KY

June 2017-June 2019

Supervisor: Bernadette Walter, Ph.D.

- Serve as first-line contact for individuals calling the community clinic, facilitating treatment services, assessment services, or providing necessary referrals 
- Collaborate with external agencies to provide referrals, outreach, and client case management

- Conduct psychotherapy intake evaluations and help guide case disposition

- Serve as first-line contact for management of crisis situations within the clinic

- Provide peer-supervision to graduate students, including assistance with intake assessments, therapy sessions, psychological testing, and clinic procedures

- Responsible for management of clinical operations, including scheduling, payment records, and chart audits; entrusted with clinic key and file room access

- Lead a group orientation for all clinical psychology graduate students to orient them to proper clinic procedures

- Attend weekly meetings with clinic director to discuss incoming clients and other clinic concerns

Military Women Psychological Services Liaison

Athena's Sisters, Louisville, KY

August 2017-May 2018

Supervisor: Bernadette Walter, Ph.D.

- Provide supportive therapeutic services during monthly meetings for a military and veteran women's non-profit support organization

- Support the organization leaders by providing psychoeducation and resources

Cognitive Behavioral Therapy- Anxiety Research and Treatment Team

Noble H. Kelley Psychological Services Center, Louisville, KY

August 2015-August 2017

Supervisor: Janet Woodruff-Borden, Ph.D.

- Conducted therapy intake evaluations for adults and children with anxiety disorders and related symptoms; wrote diagnostic evaluations and evidence-based conceptualizations

- Learned evidence-based treatment protocols and administered cognitive behavioral treatments for disorders including: GAD, SAD, selective mutism, OCD, MDD, PTSD, and Panic Disorder

- Weekly group and individual supervision, peer consultation, audio/digital recording and live observations, and case conceptualization

- Treatment modalities: cognitive-behavioral

Global Health Initiative Refugee Mental Health Clinic

University of Louisville Hospital, Louisville, KY

January 2016-July 2016

Supervisors: Monnica Williams, Ph.D., ABPP and Eric Russ, Ph.D.

- Administered and interpreted psychodiagnostic assessments in English and Spanish to newly arrived refugees

- Provided feedback to medical providers in the clinic as to best practices when working with patients who have experienced trauma in a culturally sensitive manner

Family Support Bilingual Navigator

Imagine!, Lafayette, $\mathrm{CO}$

July 2013-June 2015 
- Assisted families of children with developmental disabilities in navigating services and accessing funding

- Intervened in crisis situations including finding temporary housing and placements for children

- Worked collaboratively with other social workers, health providers, and school systems to ensure proper treatment and accommodations

- Served as a Spanish language interpreter for assessment of a client's functional abilities and eligibility for Medicaid waivers

\section{TEACHING EXPERIENCE}

Trainer-ACEs and Trauma-Informed Care

Retreat for Methodist Specialty Hospital, San Antonio, TX

December 2019

- Provided a 2-hour training on adverse childhood experiences and trauma-informed care to behavioral health providers employed by a local hospital

- Trainees included: psychiatrists, social workers, licensed professional counselors, and recreation counselors

Co-Facilitator- Workshop: Nuts and Bolts of Brief Motivational Interviewing

Texas Health Literacy Conference, San Antonio, TX

October 2019

Presenter: Stacy Ogbeide, Psy.D.

- Assisted presenter in leading group activities and provided feedback to healthcare workers in their practice of MI skills

- Provided relevant case examples to demonstrate effective use of MI skills

Co-Instructor

Course: Clinical Interviewing for Graduate Students

University of Louisville, Louisville, KY

Summer 2017, Summer 2018

Instructor: Barbara Stetson, Ph.D.

- Led instruction for first year clinical psychology graduate students on how to conduct an intake interview

- Used a clinical interviewing textbook to guide discussion and graded student assignments from the readings

- Demonstrated clinical skills using role-play and provided feedback to students on their clinical skills

- Supervised the capstone full intake interview conducted by the learning graduate student

\section{Co-Instructor}

Course: Intellectual and Cognitive Assessment

University of Louisville, Louisville, KY

Spring 2018, Spring 2019

Instructor: Bernadette Walter, Ph.D.

- Instructed clinical psychology graduate students on use of intellectual and cognitive assessment tools, including demonstrations, role-playing, and live feedback

- Supervised, graded, and provided feedback for graduate students' first administration of the WAIS-IV and WISC-V 


\section{Guest Lecturer}

Course: Life Span Development

University of Louisville, Louisville, KY

May 2018

Instructor: Catherine McDermott, M.S.

- Guest lectured for an undergraduate psychology course on anxiety research in children and adolescents

Undergraduate Assistant Teaching Assistant

Course: Deviance in U.S. Society

University of Colorado, Boulder, CO

Fall 2012

Instructor: Patti Adler, Ph.D.

- Worked with the professor and a graduate student to provide instruction for a large lecture hall class and a weekly recitation

- Provided individual and group study sessions

- Kept updated grading records for approximately 50 students

- Graded student essay exams

\section{RESEARCH EXPERIENCE}

Anxiety and Stress in Kids (ASK) Lab

University of Louisville, Louisville, $\mathrm{KY}$

Advisor: Janet Woodruff-Borden, Ph.D.

2016-2019; Graduate Research Assistant

- Produced, submitted, and had approved an IRB application investigating the role of cultural constructs on anxiety and worry in adults

- Administered the ADIS-IV-C/P and the ADIS-5-Client; wrote diagnostic evaluations for children based on data obtained from structured interviews and assessment measures; provided feedback and recommendations to parents

- Conducted data cleaning and analysis using SPSS, AMOS, and REDCap; Collected physiological data including heartrate variability, respiration, and eye movement

\section{Center for Mental Health Disparities Lab}

University of Louisville, Louisville, KY

Advisor: Monnica Williams, Ph.D.

2015-2016; Graduate Research Assistant

- Conducted reviews of literature on depressive symptoms, OCD, and race-related stress resulting in published articles and book chapters

- Collaborated with researchers at other universities to conduct a multi-site study on a new measure

- Co-led a focus group study on racial microaggressions; trained in qualitative research methods; coded and analyzed data from focus groups

- Participated the development of a new psychological services clinic within a broader refugee health clinic; collected data on a commonly used refugee mental health screener for validation in the Cuban population

- Assisted the guest editor of a special edition of Current Psychiatry Reviews in finding reviewers and compiling reviews and edited manuscripts 
- Assisted in community presentation on cultural competency in research and clinical work

\section{Arch Lab}

University of Colorado, Boulder, CO

Advisor: Joanna Arch, Ph.D.

2013 - 2014; Undergraduate Research Assistant

- Served as a facilitator in a research study of social anxiety and ACT interventions

- Assisted with data entry and cleaning including heart rate variability and questionnaire data

\section{LEADERSHIP POSITIONS}

\section{Supervisor of Intern}

August 2019-Present

- Provide daily, live supervision and feedback of master's level counseling intern within a family health primary care clinic

- Serve as primary evaluator of intern's skills, including completing formal evaluations for intern's training program

- Conduct weekly individual supervision meeting

- Develop a learning plan tailored to intern's individualized needs and goals

\section{Supervisor of Family Medicine Residents}

August 2019-Present

- Provide live supervision of family medicine physician residents during their behavioral health rotation within a family health clinic

- Provide feedback on their communication with patients, completion of behavioral health-focused interviews, and brief interventions

\section{Colloquium Coordinator}

Fall 2018- Spring 2019

- Sought and communicated with behavioral health professionals in the community to coordinate a colloquium series designed to expand upon student and faculty knowledge of topics including psychopharmacology, specialized treatments, advocacy, and many others

- Collected and organized materials, such as presentation aims and assessment measures, required by APA in order to provide faculty with continued education credits

\section{Clinical Peer Supervisor}

June 2017-June 2019

- Provided clinical supervision to graduate student clinicians in a community clinic setting, including training on specific treatment modalities and assessment procedures

- Weekly meetings to provide individual peer supervision

\section{Student Representative for Clinical Psychology Graduate Students}

July 2017-June 2019

- Elected by peers as student representative and advocate to the faculty

- Served as point of communication between students and the director of clinical training 
- Attended monthly faculty meets to verbalize students' interests and concerns

- Created, coordinated, and analyzed two program-wide student and alumni feedback surveys and presented results to the faculty with suggested solutions to identified issues

- Facilitated student applicant interviews and related activities

- Hosted welcome events for arriving graduate students

Supervisor for Undergraduate Research Assistants

August 2017-December 2018

- Provided oversight of research lab undergraduate assistants including assigning tasks, providing training, and providing career guidance

- Trained undergraduate research assistants in research practices and collaborated with them on research projects

\section{PUBLICATIONS}

Mier-Chairez, J., Arellano, B., Tucker, S., Marquez, E., \& Hooper, L. M. (2019). Theoretical, empirical, and practice literature on language brokering: Family, academic, and psychological outcomes. Journal of Human Behavior in the Social Environment, 29, 840-860.

Kuzynski, A.M., Kanter, J.W., Wetterneck, C.T., Olaz, F.O., Singh, R.S., Lee, E.B. ... MierChairez, J. ... \& Corey, M.D. (2019). Measuring intimacy as a contextual-behavioral process: Psychometric development and evaluation of the Awareness, Courage, and Responsiveness Scale. Journal of Contextual Behavioral Science.

Arellano, B., Mier-Chairez, J., \& Hooper, L.M. (2018). Parentification and language brokering: An exploratory study of the similarities and differences in their relations to continuous and dichotomous clinical mental health outcomes. Journal of Mental Health Counseling, 40, 353-373.

Williams, M.T., Mier-Chairez, J., \& Peña, A. (2017). Tools for Treating Obsessive Compulsive Disorder among Latinos. In L.T. Benuto (Ed.), Toolkit for Counseling Spanish-Speaking Clients. Springer. ISBN: 978-3-319-64880-4

Williams, M. T., Peña, A., \& Mier-Chairez, J. (2017). Assessing and Treating Racism-Related Stress and Trauma among Latinos. In L.T. Benuto (Ed.), Toolkit for Counseling SpanishSpeaking Clients. Springer. ISBN: 978-3-319-64880-4

Bosson, R., Williams, M. T., Lippman, S., Carrico, R., Kanter, J., Peña, A., Mier-Chairez, J., \& Ramirez, J. (2017). Addressing refugee mental health needs: From concept to implementation. The Behavior Therapist, 40, 110-112.

Hooper, L.M., Mier-Chairez, J., Mugoya, G., \& Arellano, B. (2016). Depressive symptoms and depression care in Latino/a adolescents: A brief review. Current Psychiatry Reviews, 12, 150162.

Williams, M. T. \& Mier-Chairez, J. (2016). Editorial: Differences in psychopathology across ethnicity and culture. Current Psychiatry Reviews, 12, 106-108.

\section{MANUSCRIPTS IN PREPARATION}

Mier-Chairez, J., Calebs, B., \& Woodruff-Borden, J. (in preparation). The role of fatalism in anxiety and worry in the Latinx population.

Williams, M.T., Kanter, J.W., Skinta, M., Martin-Willet, R., Mier-Chairez, J., Debreaux, M., \& Rosen, D.C. (in preparation). After Pierce and Sue: New research and revising the racial microaggressions taxonomy. 
Mier-Chairez, J., Calebs, B., \& Woodruff-Borden, J. (2018, November). Fatalism and its Association with Anxiety and Worry in Latinx and Non-Latinx White Adults: A Mechanical Turk Study.

Presented at the Association of Behavioral and Cognitive Therapies Annual Convention, Washington, D.C.

Mier-Chairez. J., Arellano, B., Tomek. S., \& Hooper, L.M. (2018, July). Parentification and Language Brokering: Similarities and Differences in Psychological Outcomes. Paper presentation presented at the American Psychological Association Division 45 Society for the Psychological Study of Culture, Ethnicity, and Race Research conference, Austin, TX.

\section{CONFERENCE POSTER PRESENTATIONS}

Mier-Chairez, J. \& Woodruff-Borden, J. (2020, March). Belief in Fate can Protect against Anxiety for Latinx Adolescents. Posted to be presented at the Anxiety and Depression Association of America, San Antonio, TX.

Mier-Chairez, J., Richards, A.E., Gramszlo, C., Geronimi, E.M.C., \& Woodruff-Borden (2018, November). Cognitive Features Associated with Worry among Low Income African-American Youth. Poster accepted to the Association of Behavioral and Cognitive Therapies Annual Convention, Washington, D.C.

Lee, A.H., Mier-Chairez, J., \& Woodruff-Borden, J. (2017, November). Investigating the Influence of Socioeconomic Status on Family Functioning in Families with Preschool Children. Poster presented at the Association of Behavioral and Cognitive Therapies Annual Convention, San Diego, CA.

Arellano, B., Gramszlo, C., Mier-Chairez, J., Lee, A.H., \& Woodruff-Borden, J. (2017, April). Emotional control and child anxiety: The moderating role of supportive emotion socialization. Poster presented at the Anxiety and Depression Association of America conference, San Francisco, CA.

Debreaux, M.L., Mier-Chairez, J., Heckard, D., Arney, E., Davis, D., Kanter, J., Skinta, M., \& Williams, M.T. (2016, October). "You're smart for a Black guy": A qualitative analysis of experiences of racial microaggressions in African American college students. Poster session presented at the Association for Cognitive and Behavioral Therapies conference, New York, NY.

Peña, A., Mier-Chairez, J., Bosson, R., Exposito, A., Ford, R., Diaz, Y., Davis, D., Tellawi, G., Sawyer, B., Debreaux, M., Carrico, R., Rashid, S., Williams, M., Frazier, V. (2016, September). Validation of the refugee health screener (RHS-15) for Cuban refugees/entrants. Poster session presented at the National Latina/o Psychological Association biennial conferencia, Orlando, FL.

Kuczynski, A. M., Epistola, J., Mier-Chairez, J., Stowe, T., Lee, E., Singh, S., Flaherty, B. P., Dudek, J. E., Tsai, M., \& Kanter, J. W. (2016, June). Measuring awareness, courage, and love as a contextual behavioral interpersonal process model for the development of intimacy. Poster session presented at the Association for Contextual Behavioral Science 14th Annual World Conference, Seattle, WA.

Mier-Chairez, J., Hooper, L.M., \& Mugoya, G. (2015, November). Depressive symptoms and depression care in Latino/a adolescents: A brief review. Poster session presented at the Association for Cognitive and Behavioral Therapies conference, Chicago, IL.

Debreaux, M., Mier-Chairez, J., Sawyer, B., Skinta, M., Kuczynski, A., Kanter, J., \& Williams, M.T. (2015, November). Reducing racial microaggressions: Focus group background and conceptualizations. Poster presented at the Association for Cognitive and Behavioral 
Therapies conference, Chicago, IL.

PROFESSIONAL ACTIVITIES
National Latina/o Psychological Association, Reviewer for the 2018 National Conference abstract submissions

International OCD Foundation Bilingual Program Committee, Member of the planning committee for the 2016 IOCDF conference bilingual program

Peer Reviewer: Journal of Mental Health Counseling (2018), Journal of Health Psychology (2017)

\section{COMMUNITY PRESENTATIONS}

Community Lecture, Stress and Coping, lecture for undergraduate university students in the Summer Health Professions Education Program by the Office of Diversity \& Inclusion $(2017,2018)$

Community Lecture, Helping Children with Anxiety, lecture provided to local school counselors (2017)

Community Lecture, Stress and Coping, lecture for high school students participating in Gaining Early Awareness and Readiness for Undergraduate Programs (2017)

\section{CULTURAL COMPETENCY}

Native fluency in English and Spanish

Bilingual and bicultural assessment and therapy experience

- Completed assessments for clients requesting memory evaluations for immigration applications and completed N-648 Medical Certification for Disability Exception documentation

Partnered in translating the ADIS-5 into Spanish

Assisted in translating a department clinic intake interview into Spanish

Coordinated multicultural training for graduate student clinicians

Operation Immersion

- Attended a four-day immersion training for behavioral health and other health care providers to obtain training in military culture and issues unique to service members, veterans, and their families

- Hosted by the Kentucky Army National Guard

Safe Zone training

- Participated in a two-series workshop for clinicians designed to provide the tools and resources needed to understand LGBTQ+ clients

\section{REFERENCES:}

1. Janet Woodruff-Borden, Ph.D.

Executive Vice Provost for Academic Affairs

Professor, Department of Psychology

University of Oregon

Eugene, OR 97403

(541) 346-2800

janetw@uoregon.edu 
2. Stacy Ogbeide, Psy.D., MS, ABPP, CSOWM

Associate Professor, Department of Psychiatry

Director of Behavioral Health Education, Family Medicine Residency

UT Health San Antonio

San Antonio, TX 78229

(210) 358-3695

ogbeide@uthscsa.edu

3. Cindy McGeary, Ph.D., ABPP

Associate Professor, Department of Psychiatry

Director of Clinical Psychology Internship Program

UT Health San Antonio

San Antonio, TX 78229

(210) 562-6721

mcgearyc@uthscsa.edu 